\title{
LA DIFFUSIONE DEL MARMO PROCONNESIO NELLE MARCHE IN ETÀ CLASSICA E PALEOCRISTIANA: IL RUOLO DEL PORTO DI ANCONA \\ CLAUDIA BARSANTI, ANDREA PARIBENI
}

\author{
UDC: 339.166.2:552.4(262.2:450.57)"01/05" \\ 730.033 .2 \\ Original scientific paper \\ Manuscript received: 08. 11. 2015. \\ Revised manuscript accepted: 15. 03. 2016. \\ DOI: 10.1484/J.HAM.5.111343
}

\author{
C. Barsanti \\ Università di Roma Tor Vergata \\ Dipartimento di Beni Culturali, Musica e Spettacolo \\ Via Columbia 1 - o0133 Roma, Italia \\ A. Paribeni \\ Università degli Studi di Urbino Carlo Bo \\ Dipartimento di Studi Umanistici \\ Via Bramante 16 - 61029 Urbino (PU), Italia
}

As marble exportation from Proconnesian quarries and ergasteria in roman and byzantine periods was widespread in the entire Mediterranean basin, the Adriatic coasts were affected too by a massive flow of marble either as raw material or completed sculpture. In the Marche region arrival of materials from Marmara quarries dating from $2^{\text {nd }}$ to $6^{\text {th }}$ century was encouraged by imperial (Trajan Arch at Ancona) and high ecclesiastical (S. Ciriaco) patronage, but in a broader perspective, the presence of Proconnesian marble depended on autonomous economic and trade relations that Ancona and other Marche ports were able to entertain with the production centers of the Marmara, the Aegean and the eastern Mediterranean Sea.

Keywords: marble trade - Proconnesian quarries - byzantine sculpture

In memoria di Mons. Ermanno Carnevali

Fino a non molti anni fa le informazioni circa l'impiego del marmo proconnesio in età preromana riposavano essenzialmente sulle incidentali citazioni di Vitruvio e di Plinio a proposito dei rivestimenti parietali del palazzo di Mausolo ad Alicarnasso, cui si aggiungevano isolati manufatti, come la statua di kouros del Museo Archeologico di Istanbul, in base ai quali si poteva postulare una sporadica fruizione di questo marmo già dalla fine del VI secolo a.C. ${ }^{1}$ Grazie a recenti indagini di tipo archeometrico e diagnostico, possiamo ora affermare che la platea di questi primi avvistamenti si è significativamente ampliata: sono in proconnesio, ad esempio, i sarcofagi trovati poco più di vent'anni fa nel tumulo di Kızöldün a Gümüşçay, area corrispondente grosso modo a quella della battaglia del Granico, tra i quali primeggia quello di Polyxena, databile al 520-500 a.C., ora conservato al Museo Archeologico di Çanakkale ${ }^{2}$, e più antica di un paio di decenni dovrebbe essere la stele da Sigeion, nella Troade, regione che in questo modo parrebbe imporsi come un'area privilegiata per la più precoce circolazione di manufatti in proconnesio 3 . Scendendo lungo il crinale dell'età ellenistica le attestazioni del marmo si moltiplicano in letteratura e nei rilevamenti diagnostici ${ }^{4}$, soprattutto per quanto concerne l'ambito microasiatico e per committenze di grande rilevanza, segno di un'attività estrattiva non più sporadica ma già intensa e costante.

Da questi primi traffici appaiono esclusi il Mediterraneo occidentale e l'Italia, fintanto che, con il monopolio imperiale delle cave, i marmi bianchi greco orientali man mano andranno diffondendosi in misura sempre più concorrenziale rispetto a quelli lunensi. In particolare per il proconnesio le prime limitate attestazioni a Roma e nell'Italia centro meridionale coincidono con l'età flavia e domizianea, come dimostrano gli esempi dalla domus Flavia del Palatino e dalla villa di Domiziano a Castelgandolfo ${ }^{5}$ e soprattutto quelli in

\footnotetext{
${ }^{1}$ Le indicazioni di Vitruvio, II.8.10 (cum Proconnensio marmore omnia haberet ornata) e Plinio, 36.6 (Halicarnasi domus Mausoli proconnesio marmore exculta est latericiis parietibus) vengono confermate, tra gli altri, da T. CRAMER, K. GERMANN, W.-D. HEILMEYER, Petrographic and Geochemical Characterization of the Pergamon Altar (Telephos Frieze) Marble in the Pergamon Museum, Berlin, in L. Lazzarini (ed.), Interdisciplinary Studies on Ancient Stones, ASMOSIA VI. Proceedings of the Sixth International Conference of the «Association for the study of marble and other stones in Antiquity», Venice,June 15-18 2000, Padua, 2002, p. 285-291. Sul kouros del Museo Archeologico di Istanbul vedi G. MENDEL, Catalogue des sculptures grecques, romaines et byzantines, Constantinople, II, 1913, n. 517, pp. 214-217.

${ }^{2}$ N. SEVINC,, A New Sarcophagus of Polyxena from the Salvage Excavations at Gümüscçay, in Studia Troica, 6, 1996, p. 251-264; J. ZÖLDFÖLDI, M. SATIR, Provenance of white marble building stones in the monuments of ancient Troia, in G.A. Waer, E. Pernicka, H.-P. Uerpmann (eds.), Troia and the Troad: scientific approaches, Berlin, 2003, p. 203-222; CH. B. ROSE, The Archaeology of Greek and Roman Troy, Cambridge Univ. Press, 2014, p. 100 e 143, nota 308; F. CROISSANT, Observations sur le sarcophage de Polyxène et les styles de l'Ionie du Nord à la fin de l'Archaïsme, in Revue Archéologique, $2015 / 2$, p. 259-292. Un altro sarcofago in proconnesio databile all'ultimo quarto del IV a.C. proveniente da Can, con rilievi di scene di battaglia e venatorie su cui si conservano tracce di finiture dipinte eccezionalmente conservate, è segnalato in CH. B. ROSE, Ilion, in W. Radt (ed.), Stadtgrabungen und Stadtforschung in westlichen Kleinasien: Geplantes und Erreichtes, Internationalen Symposion 6./7. August 2004 in Bergama -Türkei, (Byzas, 3), Istanbul, 2006, p. 135-158, in part. p. 143-146. 3 Sulla stele di Sigeion vedi S. WALKER, The marble quarries of Proconnesos: isotopic evidence for the age of the quarries and for the lenos-sarcophagi carved at Rome, in P. Pensabene (ed.), Marmi Antichi. Problemi d'impiego, di restauro e d'identificazione, ("StMisc" 26), Roma, 1983, p. 57-68, in part. p. 58, tav. 1,1. Sulla scoperta della eccezionale epigrafe e sulle successive vicissitudini antiquariali rinvio a F. THOMASSON, Justifying and Criticizing the Removals of Antiquities in Ottoman Lands: Tracking the Sigeion Inscription, in International Journal for Cultural Property, 17, 2010, p. 493-517; B. ANDERSON, 'An Alternative Discours': Local Interpreters of Antiquities in the Ottoman Empire, in Journal of Field Archaeology, 40, 2015, p. 450-460, in part. p. 452.

${ }^{4}$ T. CRAMER, K. GERMANN, W.-D. HEILMEYER, Petrographic, op. cit. (n. 1), per quanto concerne le analisi petrografiche effettuate su alcuni campioni del fregio di Telefo ora al Pergamon Museum.

${ }^{5}$ P. PENSABENE, I marmi nella Roma antica, Roma, 2013, p. 320, con bibliografia precedente. Una recente acquisizione è costituita dal frammento di statua colossale di Domiziano dal Palatino (inv. n. 515204), coerente con il frammento di un dito trovato negli scavi Rosa (inv. n. 515245) che è risultata essere in marmo proconnesio, cfr. Divus Vespasianus. Il bimillenario dei Flavi, a cura di F. Coarelli, Milano, 2009, catalogo n. 33 , p. 442.
} 


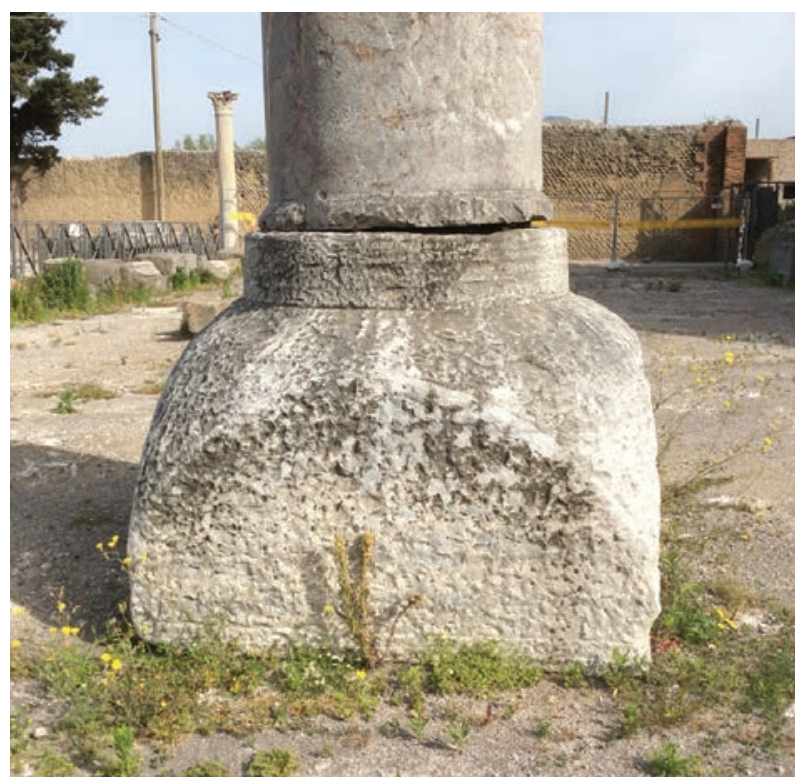

Fig. 1. Pompei, tempio di Venere, capitello semilavorato in marmo proconnesio (foto Pardi).

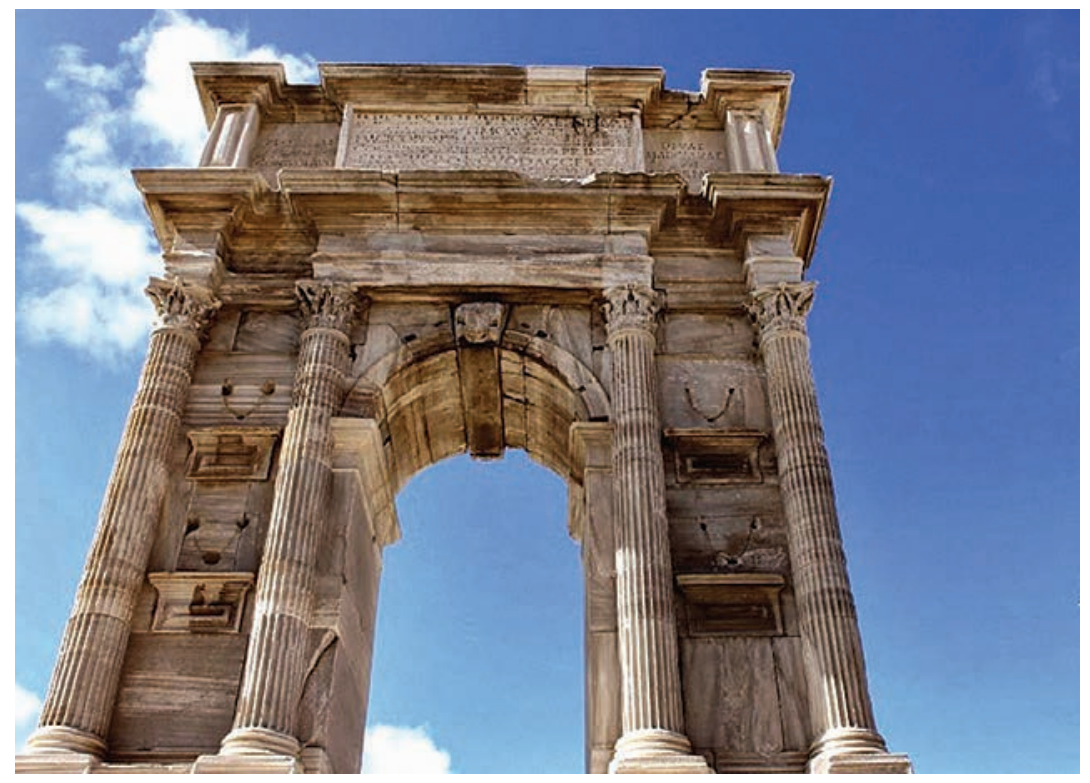

Fig. 2. Ancona, Arco di Traiano. area campana: a Pozzuoli con i capitelli e le colonne dell'anfiteatro maggiore ${ }^{6}$ e a Pompei con i marmi semilavorati (un capitello, una base e un blocco con tracce di segagione) presso il tempio di Venere (fig. 1), ad ogni evidenza da riconoscere come una scorta ivi accumulata per i restauri da farsi dopo il terremoto del 62 d.C.7. In generale gli studiosi concordano comunque nel ritenere che solo nell'inoltrato II secolo le importazioni di marmo proconnesio a Roma e più in generale in Occidente assumeranno una effettiva consistenza, facendosi largo anche nelle grandi opere pubbliche, per poi sbaragliare il campo in età severiana ${ }^{8}$.

Questo quadro così delineato non pone, a mio parere, nel risalto che loro compete, alcune precoci attestazioni del marmo proconnesio in area marchigiana: si è detto, ed è vero, dell'assenza in Italia di questo litotipo prima dell'età alto imperiale, ma un'importante, anche se isolata, eccezione è fornita da una stele funeraria ellenistica proveniente dalla necropoli di Ancona che, nel quadro di un'analisi di laboratorio comparata con altre analoghe stele recentemente eseguita da Fabrizio Antonelli e Lorenzo Lazzarini, è risultata essere di marmo proconnesio ${ }^{9}$, area cui rimanda del resto anche l'analisi stilistica ed iconografica del pezzo che trova puntuali confronti con materiali da Bisanzio ${ }^{10}$. Oltre a costituire allo stato attuale delle nostre conoscenze il primo caso di importazione di proconnesio in Italia, questa stele, assieme alle altre di marmo pario e lesbio dalle necropoli anconetane, testimonia dell'ampiezza e dell'articolazione dei rapporti economici e culturali che Ancona intratteneva con il mondo greco orientale tra III e I secolo a.C.

Anche nel quadro delle presenze in Italia del nostro marmo in età imperiale, Ancona giuoca un ruolo importante, addirittura in anticipo rispetto alla capitale. L'Arco di Traiano (fig. 2), dedicato nel 114-115 d.C. in onore dell'imperatore per celebrare le sue provvidenze a favore della città - ma

${ }^{6}$ F. DEMMA, Anfiteatro Flavio di Pozzuoli, la decorazione marmorea nella storia edilizia: tipologia e stile; restauri; officine ed artigiani, in Archaeological Methods and Approaches: Ancient Industry and Commerce in Italy (British Archaeological Reports - International Series, 1262) Oxford, 2004, p. 243-261.

7 P. PENSABENE,L. IACOBELLI, La decorazione architettonica del tempio di Venere a Pompei. Contributo allo studio e alla ricostruzione del santuario, in Rivista di Studi pompeiani, 7, 1995-96, p. 45-75, in part. p. 69-72, fig. 52-54; M. CARROLL, G. MONTANA, L. RANDAZZO, R. GIARRUSSO, Recovering Evidence for the use of marble and coloured limestone in the first Century A.D., in excavations at the sanctuary of Venus at Pompeii, in The Journal of Fasti on Line 2008, p. 1-13. Sempre in proconnesio è un piano di tavolo nella casa dei Vettii, cfr. J. CLAYTON FANT, S. CANCELLIERE, L. LAZZARINI, M. PREITE MARTINEZ, B. TURI, White Marble at Pompeii: sampling the Casa dei Vettii, in L. Lazzarini ASMOSIA VI, op. cit. (n. 1), p. 309-315, in part. p. 311, fig. 2, p. 313-314. Per la concessione della fotografia del capitello semilavorato ringrazio la dott.ssa Roberta Pardi della Soprintendenza Pompei. Che per il proconnesio gli esempi sopra citati costituiscano le prime e isolate attestazioni in area campana lo conferma il fatto che di questo tipo di marmo non si trova traccia nei thermopolia di Pompei ed Ercolano, recentemente oggetto di un capillare censimento dei marmi utilizzati dal punto di vista quantitativo e qualitativo: cfr. J. C. FANT, B. RUSSELL, S. J. BARKER, Marble Use and Reuse at Pompeii and Herculaneum: the Evidence from the Bars, in Papers of the British School at Rome, 81, 2013, pp. 181-209.

${ }^{8}$ Cfr. F. VAN KEUREN, D. ATTANASIO, J. J. HERRMANN JR., N. HERZ, L. P. GROMET, Multimethod Analyses of Roman Sarcophagi at the Museo Nazionale Romano, Rome, in J. Elsner, J. Huskinson (eds.), Life, Death and Representation. Some New Work on Roman Sarcophagi (Millennium-Studien 29), Berlin New York, 2010, p. 149-187, in part. p. 168-169 per alcune considerazioni sulla progressiva affermazione del proconnesio sul mercato del marmo nella seconda metà del II secolo d.C., tanto nella produzione dei sarcofagi quanto nellimpiego del litotipo per le maggiori committenze pubbliche di ambito urbano. In questo senso le attestazioni di marmo proconnesio in monumenti della prima età imperiale vengono solitamente interpretate come restauri e integrazioni di età adrianea e antonina D. ATTANASIO, M. BRILLI, M. BRUNO, L. UNGARO, M. VITTI, Rome: White Marbles in the Fora of Caesar, Augustus and Trajan, in A. Gutierrez Garcia, M. Pilar-Lapuente, I. Rodà (eds.), Interdisciplinary Studies on Ancient Stones, Proceedings of the IX ASMOSIA Conference (Tarragona 2009), Tarragona, 2012, p. 331-343, in part. p. 336.

9 F. ANTONELLI, L. LAZZARINI, L'identificazione dei marmi delle stele della necropoli di Ancona (Marche, Italia), e la presenza del marmo nell'alto Adriatico in età ellenistica, in Marmora, 7, 2011, p. 37-53, in part. p. 45-46, fig. 1.d (S11); F. ANTONELLI, L. LAZZARINI, The Use of White Marble in the Central and Upper Adriatic between Greece and Rome: Hellenistic Stelae from the Necropolis of Ancona (Italy), in Cambridge Archaeological Journal, 23.2, 2013, p. 149-162, in part. p. 157 .

${ }^{10} \mathrm{~F}$. COLIVICCHI, Dal pallium alla toga: Ancona tra ellenismo e romanizzazione, in Ostraka, IX, 2000, p. 135-142, in part. p. 137-138, fig 2.a. ove si potranno trovare i confronti con stele funerarie prodotte nell'area di Bisanzio. 
probabilmente già eretto intorno al 100 d.C. con diversi partiti decorativi - è una struttura interamente in marmo, costituita da trenta blocchi con quattro coppie di colonne che fiancheggiano l'unico fornice, un'architettura - già definita "nella severa semplicità della sua forma, gioconda e sorridente" - forse derivante da un progetto di Apollodoro di Damasco" ${ }^{\text {. }}$. In letteratura il marmo è tradizionalmente ritenuto essere imezio, sulla base di un superficiale esame autoptico e delle convenzionali quanto fuorvianti nomenclature delle due specie marmoree che erano state adottate in passato ${ }^{12}$. Sulla base di analisi combinate (spettroscopia EPR e studio degli isotopi) condotte poco più di dieci anni fa su un lotto di campioni prelevati dall'arco approfittando dei ponteggi in opera per i restauri, si è potuto stabilire in maniera inequivocabile che il marmo non è imezio, come comunemente si riteneva, bensì proconnesio ${ }^{13}$; l'indagine ha inoltre evidenziato che i blocchi sono stati prelevati da diverse aree di estrazione dell'isola di Marmara, dimostrando con ciò che già all'epoca le cave operavano in una modalità industriale, per soddisfare le richieste diversificate della committenza pubblica esemplificate, oltre che dallo stesso arco di Ancona, anche da grandi monumenti microasiatici quali, per fare un esempio, il coevo Traianeum di Pergamo ${ }^{14}$.

Va detto però che, nel prosieguo della piena e tarda età imperiale, non si ravvisano ad Ancona ed in altri centri romani delle Marche presenze di marmo proconnesio paragonabili, per quantità e prestigio della committenza, al caso emblematico dell'arco di Traiano. Alcune recenti ricognizioni delle provenienze e delle tipologie di marmi bianchi e colorati attestati in siti quali Urbs Salvia, Forum Sempronii, Pitinum
Pisaurense e Pollentia, hanno evidenziato percentuali in alcuni casi significative di manufatti in proconnesio, pertinenti per lo più a sculture in funzione architettonica non riferibili però a contesti unitari e anzi spesso reimpiegati in monumenti di età medievale e moderna ${ }^{15}$. Anche nell'ambito della produzione dei sarcofagi, la classe di materiali che forse maggiormente assicurò alle fabbriche dell'isola di Marmara il monopolio nel commercio dei marmi a partire dal II secolo, si possono segnalare nelle Marche pochi e isolati casi: mi limito a quello del sarcofago con fronte strigilata e lati corti con decorazioni di scuta incrociati, databile alla fine del II d.C., reimpiegato come cassa per il corpo di San Romualdo nell'Abbazia di San Salvatore a Valdicastro, in provincia di Ancona, per il quale non è nemmeno possibile avanzare congetture circa le modalità del suo arrivo nelle Marche, molto probabilmente mediato dalle officine di Roma nelle quali il marmo della Propontide venne lavorato ${ }^{16}$.

Dopo il rallentamento determinato dalle critiche condizioni economiche del travagliato III secolo, l'attività estrattiva e produttiva delle cave dell'isola di Marmara conobbe un rinnovato slancio grazie alla fondazione di Costantinopoli: l'isola di marmo, posta strategicamente presso la nuova capitale - anzi, come scriverà nel XV secolo il dotto Manuele Crisolora, addirittura lì sorta quasi per un provvidenziale disegno divino ${ }^{17}$ - mise a disposizione le proprie risorse per dotare di un manto rifulgente e prezioso monumenti pubblici, fori, infrastrutture, strade porticate, chiese e palazzi eretti tra IV e VI secolo nella Nuova Roma e affermare, attraverso questa veste di marmo, il ruolo di Costantinopoli quale sede del basileus e centro del potere imperiale ${ }^{18}$.

" S. DE MARIA, Gli archi onorari di Roma e dell'Italia romana, Roma, 1988, p. 227-229; M. LUNI, L'Arco di Traiano in Ancona e la riscoperta nel Rinascimento, in L. Bacchielli, M. Bonanno Aravantinos (eds.), Studi di Antichità in memoria di Sandro Stucchi (Studi Miscellanei 29), Roma, 1996, p. 197-206. La citazione riportata nel testo è presa da R. PARIBENI, Optimus princeps. Saggio sulla storia e sui tempi dellimperatore Traiano (Biblioteca Storica Principato, V), Messina, 1926, II, p. 245. Per la storia del monumento in età moderna, anche in rapporto all'impianto portuale in cui si trova, vedi S. HANKE, Monumente am Wasser. Ehrenstandbilder in Hafenanlagen der Frühen Neuzeit, in A. Nova, S. Hanke (eds.), Skulptur und Platz. Raumbesetzung, Raumüberwindung, Interaktion, Berlin, München, 2014, p. 223-250, in part. p. 227-233.

${ }_{12}$ M. E. BLAKE, Roman Construction in Italy from Nerva through the Antonines (edited and compiled by D. Taylor Bishop), American Philosophical Society, Philadelphia, 1973, p. 264-265 e 294; S. DE MARIA, Gli archi onorari op. cit. (n. 11), p. 227, con bibliografia precedente. La obliterazione nella letteratura critica tradizionale del marmo proconnesio a vantaggio dell'imezio si può far risalire addirittura a Faustino Corsi che, sulla base dell'esame olfattivo, attribuiva al più conosciuto litotipo greco tutti quei marmi caratterizzati dal forte odore tra cipolla e uovo marcio che è tipico invece anche del marmo cavato sull'isola di Marmara.

${ }^{13}$ Cfr. D. ATTANASIO, G. DE MARINIS, P. PALLECCHI, R. PLATANIA, P. ROCCHI, An EPR and isotopic study of the marbles of the Trajan's Arch at Ancona: an example of alleged Hymettian provenance, in Archaeometry, 11, 2003, p. 553-568. Per la metodica seguita nel rilevamento vedi D. ATTANASIO, M. BRILLI, M. BRUNO, The Properties and Identification of marble from Proconnesos (Marmara Island, Turkey): a new database including isotopic, EPR and petrographic data, in Archaeometry, 50.5, 2008, p. 747-774.

${ }^{14}$ T. CRAMER, K. GERMANN, W.-D. HEILMEYER, op.cit. (n. 1), p. 290. Sul Traianeum vedi H. STILLER, Das Traianeum (Altertümer von Pergamon, Bd. 5:2), Berlin, 1895 .

${ }^{15}$ F. ANTONELLI, L. LAZZARINI, B. TURI, Prime indagini di identificazione dei marmi e delle pietre del municipio romano di Pitinum Pisaurense (Macerata Feltria), in W. Monacchi (ed.), Storia e Archeologia di Pitinum Pisaurense, Urbania, 2002, p. 189-211; F. ANTONELLI, Determinazione della provenienza del marmo del cratere da Urbs Salvia. Appendice II a G. M. FABRINI, Urbs Salvia: un cratere neoattico dedicato alle ninfe', in Mare Internum, 3, 2011, p. 78-79; M. L. AMADORI, C. GORGONI, P. PALLANTE, G. RAFFAELLI, S. RINALDI TUFI, Identificazione e provenienza dei marmi: la statuaria romana nel Museo Civico Malatestiano di Fano, in I Quaderni del Museo. Rivista del Museo Civico di Fano, 3, 2012, p. 130-137; F. ANTONELLI, L. LAZZARINI, White and Coloured Marbles of the Roman Town of Urbs Salvia (Urbisaglia Macerata, Marche, Italy), in Oxford Journal of Archaeology, 32.3, 2013, p. 293-317; F. ANTONELLI, S. COLUMBU, M. LEZZERINI, D. MIRIELLO, Petrographic Characterization and Provenance Determination of White Marbles used in Roman Sculpture of Forum Sempronii (Fossombrone, Marche, Italy), in Applied Phisics A (Springer) 2013, p. 1-8; M. L. AMADORI, C. GORGONI, P. PALLANTE, Studio di provenienza dei marmi dei manufatti dalla colonia romana di Potentia, in G. Baldelli, F. Lo Schiavo (eds.), Amore per l'Antico. Dal Tirreno all'Adriatico, dalla Preistoria al Medioevo e oltre. Studi di antichità in ricordo di Giuliano de Marinis, Roma, 2014, II, p. 769-777.

${ }^{16}$ G. BARATTA, Il sarcofago romano destinato a San Romualdo nell'Abbazia di San Salvatore a Valdicastro (AN): un caso di riutilizzo, in Picus, 34, 2014, p. 43-64. Sulla produzione dei sarcofagi di età imperiale negli opifici del Proconneso, oltre al classico J.B. WARD-PERKINS, Nicomedia and the Marble Trade, in Papers of the British School at Rome, 48, 1980, p. 23-69, vedi ID., The Trade in Sarcophagi, in H. Dodge, B. Ward-Perkins (eds.), Marble in Antiquity. Collected Papers of J.B. Ward-Perkins (Archaeological Monographs of the British School at Rome. 6), British School at Rome London, 1992, p. 31-37, cui aggiungi ora B. RUSSELL, The Roman Sarcophagus 'Industry', a Reconsideration, in J. Elsner, J. Huskinson, Life, Death, op. cit. (n. 8) p. 119-147. ${ }^{17}$ M. CRISOLORA. Roma parte del cielo. Confronto tra l'Antica e la Nuova Roma, introduzione di E.V. Maltese, traduzione e note di G. Cortassa, Torino, 2000, p. 81 .

${ }^{18}$ Sulla monumentalizzazione della capitale e il ruolo rivestito dal marmo in questo processo vedi, nella vasta bibliografia C. BARSANTI, A. GUIGLIA, A. PARIBENI, Le officine dell'imperatore: marmora byzantina, in A. C. Quintavalle (ed.), Medioevo: le officine, Atti del Convegno internazionale di studi Parma, 22-27 settembre 2009, (I convegni di Parma, 12), Milano, 2010, pp. 118-151. 
Oltre ai ben noti monumenti della capitale, danno pieno riscontro di questo vertiginoso aumento della estrazione, lavorazione e distribuzione dei manufatti in marmo proconnesio i materiali semilavorati presenti in cava ${ }^{19}$, quelli, tragicamente perduti, dei relitti naufragati lungo le rotte dell'Egeo e del Mediterraneo ${ }^{20}$ e quelli tuttora presenti nelle città e nei territori beneficiati da questa disseminazione, le cui finalità, a parte quelle meramente economiche, funzionali od estetiche, erano quelle di ribadire e rafforzare, attraverso la metafora della circolazione dei marmi, i sentimenti di identità e di appartenenza che legavano centro e periferia, imperatore e sudditi: in questa dialettica, Ancona ed il suo porto ebbero, nella prima età bizantina, un ruolo non secondario.

Andrea Paribeni

Il San Ciriaco, la scenografica cattedrale di Ancona, che domina la città e il suo porto dall'alto dell'antica acropoli (fig. 3), è un monumento di grande fascino e suggestione, che esibisce con grande dignità le memorie e le spoglie del proprio passato ${ }^{21}$, tra le quali, anche un gruppo di sculture che formano un insieme omogeneo e contemporaneo e che l'ipotesi più probabile porta ad identificarle come spoglie dell'arredo architettonico della primitiva basilica paleocristiana dedicata a San Lorenzo, costruita sul volgere del V secolo sui resti dell'antico tempio di Venere Euplea ${ }^{22}$.

Per tipologia di materiale, il marmo proconnesio, e caratteristiche tecnico-esecutive, queste sculture, che possono essere a pieno titolo riconosciute come manufatti di tipo standard prodotti dagli opifici attivi presso le cave del Proconneso, facevano con ogni probabilità parte di un set di elementi appositamente confezionati per il San Lorenzo, sbarcato nel porto di Ancona da una nave proveniente da

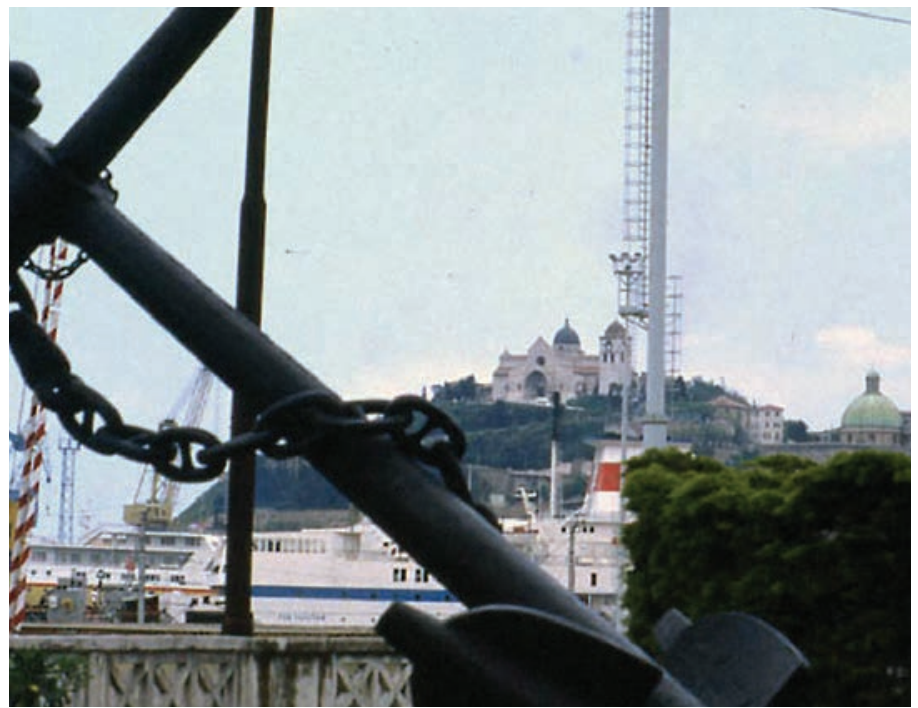

Fig. 3. Ancona, il porto e la cattedrale di San Ciriaco: veduta dall'Arco Clementino.

Costantinopoli. Nel caso specifico riterrei infatti verosimile pensare all'esito di un'importazione diretta anziché presupporne un'acquisizione indiretta tramite Ravenna, verso la quale convergeva il flusso dei marmi importati dall'area greco-costantinopolitana e dove, fin dall'età imperiale, operava un grande centro, organizzato su scala industriale, di raccolta, lavorazione e distribuzione di svariate categorie di manufatti marmorei ${ }^{23}$.

Sotto tale prospettiva le sculture reimpiegate nel San Ciriaco offrirebbero diversi spunti di riflessione sul fenomeno delle importazioni dei materiali marmorei, un fenomeno di vasta portata e di larga diffusione geografica ${ }^{24}$, esteso anchea gran parte delle regioni bagnate dall'Adriatico ${ }^{25}$. Essi costi-

${ }^{19}$ N. ASGARI, The Proconnesian Production of Architectural Elements in Late Antiquity, based on the Evidence from the Marble Quarries, in C. Mango and G. Dagron (eds.), Constantinople and Ist Hinterland, Aldershot, 1995, p. 263-288.

${ }^{20}$ E. F. CASTAGNINO BERLINGHIERI, A. PARIBENI, Byzantine Merchant Ship and Marble Trade. New Data from the Central Mediterranean, in Skyllis. Zeitschrift für Unterwasserarchäologie, 11, 1, 2011, p. 64-75; B. RUSSELL, Roman and Late-Antique Shipwrecks with Stone Cargoes: a New Inventory, in Journal of Roman Archaeology, 26, 2013, p. 331-361; E. F. CASTAGNINO BERLINGHIERI, A. PARIBENI, Marble production and marble trade along the Mediterranean coast in Early Byzantine Age ( $5^{\text {th }}$ - $6^{\text {th }}$ centuries): data from quarries, shipwrecks and monuments, in P. M. Militello, H. Öniz (eds.), SOMA 2011. Proceedings of the $15^{\text {th }}$ Symposium on Mediterranean Archaeology, held at the University of Catania 3-5 March 2011 (BAR International Series 2695), Oxford, 2015, II, p. 1033-1041.

${ }^{21}$ Per la complessa e travagliata vicenda costruttiva del monumento resta fondamentale il saggio di M. MARINELLI, L'architettura romanica in Ancona (Seconda edizione con gli aggiornamenti di G. Annibaldi e A. Boni), Ancona, 1961. Per ulteriori, più aggiornati approfondimenti, si rinvia ai contributi riuniti nel volume San Ciriaco. La cattedrale di Ancona. Genesi e sviluppo, a cura di M.L.Polichetti, Milano, 2003; si tengano presenti anche le considerazioni di P. PIVA, Marche romaniche, Milano, 2003, p. 177-193.

${ }^{22}$ C. BARSANTI, Testimonianze bizantine in Ancona. Le spoglie paleocristiane del S. Ciriaco, in Atti VI CNAC (Pesaro-Ancona, 19-23 settembre 1983), II, Ancona, 1985, p. 387-404. La specifica facies del reimpiego, variamente declinata nei monumenti medievali di Ancona, è stata presa in esame nel contesto di un intervento presentato da chi scrive in sinergia con Andrea Paribeni al convegno Il Piceno prima di Fiastra. Topografia, architettura ed arte. Giornate di Studio, San Costanzo (MC), 14-15 maggio 2010: Sculture bizantine nelle Marche centrali. Provenienza, reimpiego, nuove funzionalità: il caso del San Ciriaco di Ancona.

${ }^{23}$ Si veda la sintesi tratteggiata, limitatamente all'approvvigionamento delle colonne, da J.G. HARPER, The Provisioning of Marble for the Sixth-Century Churches of Ravenna: A Reconstructive Analysis, in R.L. Coltella et alii (ed.), Pratum Romanum. Richard Krautheimer zum 10o. Geburtstag, Wiesbaden, 1997, p. 131-148.

${ }^{24}$ A questo riguardo, cfr. C. BARSANTI, L'esportazione di marmi dal Proconneso nelle regioni pontiche durante il IV-VI secolo, in Rivista dell'Istituto Nazionale d'Archeologia e Storia dell'Arte (= RINASA) s. III, 12, 1989, p. 91-220; J.-P. SODINI, Le commerce des marbres à l'époque protobyzantine, in Hommes et richesses dans l'empire byzantine (IVe-VIIe s.), I, Paris, 1989, p. 163-186; J.-P. SODINI, C. BARSANTI, A. GUIGLIA GUIDOBALDI, La sculpture architecturale en marbre au VIe siècle à Constantinople et dans les régions sous influence constantinopolitaine, in Acta XIII CIAC (Split - Poreč 1994), Città del Vaticano, 1998, p. 301-376; J.-P. SODINI, Le commerce des marbres dans la Méditerranée (IVe-VIIe S.), in V Reuniò d'Arqueologia Cristiana Hispànica (Cartagena, 16-19 d'abriò de 1998), Barcelona, 2000, p. 423-446.

${ }^{25}$ Per un quadro d'insieme cfr. C. BARSANTI, La scultura paleocristiana e bizantina, in P. Zampetti (ed.), Scultura nelle Marche, Firenze, 1993, p. 59-70; EAD., Mosaici pavimentali paleocristiani in Ancona: alcune riflessioni, in Atti del II Colloquio dell'AISCOM (Roma, 5-7 dicembre 1994), Bordighera, 1995, p. 181-200; EAD., Un inedito pluteo costantinopolitano a Jesi, in F. Guidobaldi (ed.), Domum tuam dilexi. Miscellanea in onore di Aldo Nestori, Città del Vaticano, 1998, p. 23-53; P. PENSABENE, C. BARSANTI, Reimpiego e importazioni di marmi nell'Adriatico paleocristiano e bizantino, in La cristianizzazione dell'Adriatico (Antichità Altoadriatiche 66), Trieste, 2008, p. 455-490; Y.A. MARANO, Il commercio del marmo nell'Adriatico Tardoantico (IV-VI sec. d.C.), in S. Collodo, G.L. Fontana (eds.), Eredità culturali dell'Adriatico. Archeologia, Storia, Lingua e Letteratura, Roma, 2008, p. 159-174, in part. p. 166-167. 


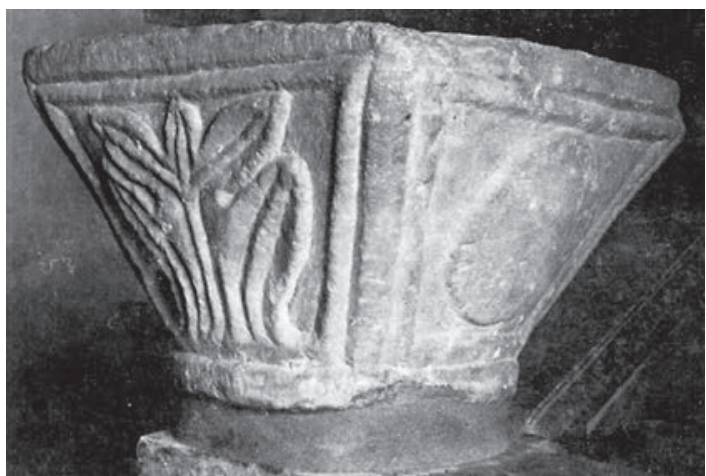

Fig. 4a. Pesaro, Biblioteca Oliveriana: capitello imposta del tipo cosiddetto 'a pannelli' (da Russo).

tuiscono oltretutto un caso decisamente paradigmatico nel panorama documentario, altrimenti rarefatto, riguardante la presenza di manufatti marmorei nelle Marche. Se si eccettua Ancona, abbiamo in effetti solo sporadiche attestazioni di materiali eterogenei, decontestualizzati, erratici, oppure reimpiegati in contesti seriori, i quali, come ho già avuto modo di sottolineare, mancando il supporto dei dati archeologici ed anche quello delle fonti storiche, sollevano reali difficoltà interpretative poiché non è possibile stabilire per essi una correlazione $a b$ antiquo con quei siti, tanto da considerarli indizi di presenze più consistenti, ma superstiti solo in minima parte; essi sarebbero pertanto delle presenze del tutto casuali e dunque ben difficilmente collegabili al fenomeno delle importazioni ${ }^{26}$.

Per alcuni di essi è stata comunque presa in considerazione anche un'eventuale provenienza dall'area ravenna$\mathrm{te}^{27}$, mentre in altri casi si tratterebbe di marmi di spoglio costantinopolitani i quali, all'indomani del sacco latino della capitale bizantina, ripresero numerosi a viaggiare, soprattutto sulle navi che percorrevano le rotte adriatiche ${ }^{28}$.

Esemplifica questo quadro documentario dai contorni estremamente elusivi un capitello imposta già nella colle- zione antiquaria del nobile Annibale degli Abbati Olivieri, ed ora collocato sul parapetto del monumentale scalone che conduce alla Biblioteca Oliveriana di Pesaro (fig. 4a) ${ }^{29}$, del quale mancano purtroppo indicazioni utili a rintracciarne la provenienza.

Il capitello, del tipo cosiddetto 'a pannelli', appartiene alla variante più semplice della categoria, di manifattura costantinopolitana, diffusa in età giustinianea ed oltre ${ }^{3^{\circ}}$, la cui struttura decorativa è caratterizzata da pannelli trapezoidali incorniciati da lisce modanature nelle quali trovano posto un disco, una foglia di vite e due schematiche foglie di acanto che parrebbero 'ritoccate'.

Nella vasta gamma dei confronti proponibili, appaiono particolarmente vicini sia un esemplare della collezione lapidaria dell'Ayasofya Müzesi', sia, soprattutto, uno tra i molti esemplari riutilizzati nella Grande Moschea di Kairouan ${ }^{32}$, il quale mostra una foglia di vite in tutto e per tutto simile, anche nella fattura piuttosto frettolosa, a quella scolpita sul capitello della Biblioteca Oliveriana (fig. 4b), il quale, vorrei soggiungere, resta del tutto isolato nel panorama adriatico dove, eccettuati i più tardi esemplari in opera nella cripta della cattedrale di Otranto e quello nel Museo di San Nicola

\footnotetext{
${ }^{26}$ Difficilmente rintracciabile è, ad esempio, l'origine del capitello di parasta del Museo Civico di Fano: F. BATTISTELLI, Silloge di reperti e sculture paleocristiane e altomedievali di Fano, in Atti VI CNAC, op.cit. (n. 22), p. 404-417, in part. p. 413, fig. 4; M.C. PROFUMO, Fano cristiana, in F. Milesi (ed.), Fano romana, Fano, 1992, p. 518; C. BARSANTI, Un inedito pluteo op.cit. (n. 25), p. 26. Sconosciuta è pure la provenienza di un pilastrino nel Museo A. Vernarecci di Fossombrone F. GUIDOBALDI, C. BARSANTI, A. GUIGLIA GUIDOBALDI, San Clemente. La scultura del VI secolo, Roma, 1992, p. 211, fig. 320; C. BARSANTI, La scultura...op.cit. (n. 25), p. 65, fig. 18; Cfr. C. BARSANTI, R. FLAMINIO, A. GUIGLIA, Corpus della scultura altomedievale. La diocesi di Roma, tomo VII, La Terza regione ecclesiastica, Spoleto 2015, p. 166

${ }^{27} \mathrm{~A}$ una bottega ravennate è stata infatti attribuita l'esecuzione del bel sarcofago di marmo proconnesio, datato ai primi decenni del VI sec., collocato in un momento non meglio precisabile nella cripta della chiesa di San Gervasio a Mondolfo, nella valle del Cesano: J. KOLLWITZ, H. HERDEJÜRGEN, Die Sarkophage der westlichen Gebiete des Imperium romanum. 2, Die ravennatischen Sarkophage, Berlin, 1979, cat. B.30, p. 78, 159-16o, tav. 82,1-2, 83, 1; R. FARIOLI, Ravenna, Costantinopoli: considerazioni sulla scultura del VI secolo, in CARB 30, 1983, p. 205-253, in part. p. 211-214, 251-252, fig. 7. C. BARSANTI, La scultura... op.cit. (n. 25), p. 65, fig. 17 ; EAD., Un inedito pluteo op.cit. (n. 25), p. 26-27 e nota 17. Una provenienza dalla medesima area è stata ipotizzata anche per la coppia di plutei marmorei riutilizzati nel portale della chiesa di Santa Maria di Chiaravalle della Castagnola: C. BARSANTI, Santa Maria di Chiaravalle della Castagnola: un'abbazia cistercense tra leggenda e realtà, in Arte d'Occidente, temi e metodi. Studi in onore di Angiola Maria Romanini, Roma, 1999, p. 391-403.

${ }^{28}$ È forse questo il caso del pluteo riutilizzato nel timpano del portale trecentesco della chiesa di San Marco a Jesi: C. BARSANTI, Un inedito pluteo...op.cit (n. 25), p. 23-53; C. BARSANTI, I plutei degli intercolumni delle gallerie, in A. Guiglia Guidobaldi, C. Barsanti (eds.), Santa Sofia di Costantinopoli. L'arredo marmoreo della Grande Chiesa giustinianea, Città del Vaticano, 2004, p. 473, fig. 252.

29 E. RUSSO, Testimonianze monumentali di Pesaro dal secolo VI all'epoca romanica, in Pesaro tra Medioevo e Rinascimento (Pesaro II), Venezia, 1989, p. 79-147, in part. p. 133-134, fig. 65-66 (pur riconoscendo strette affinità con i capitelli di VI secolo, viene cautamente preferita una datazione all'XI secolo); C. BARSANTI, La scultura... op.cit. (n. 25), p. 65; EAD., Un inedito pluteo...op.cit. (n. 25), p. 25.

${ }^{30}$ Si veda soprattutto J. KRAMER, Kämpferkapitelle mit den Monogrammen Kaiser Justinus II. und seiner Gemahlin, der Kaiserin Sophia in Yalova Kaplıcaları (Termal), in M. Restle (ed.), Festschrift für Klaus Wessel zum 7o. Geburtstag (in memoriam), München, 1988, p. 175-190; ed inoltre C. BARSANTI, L'esportazione...op.cit. (n. 23), p. 181; EAD., Materiali bizantini poco noti o inediti della Bitinia, in CARB 42, 1995, p. 47-69, in part. p. 6o-61; e, da ultima, A. GUIGLIA, Le sculture bizantine di Trebisonda: appunti di viaggio, in RINASA 66, 2015, p. 131-150, in part. p. 137-138.. Per la diffusione postgiustinianea dei capitelli imposta 'a pannelli', variamente declinati in epoca medio bizantina, si rinvia a M. DENNERT, Mittelbyzantinische Kapitelle, Bonn, 1997, p. 32-48, tav. 13-19. ${ }^{31}$ C. BARSANTI, A. GUIGLIA, The Sculptures of the Ayasofya Müzesi in Istanbul, Istanbul, 2010, p. 93, fig. 100.

${ }^{32}$ N. HARRAZI Chapiteaux de la grande Mosquée de Kairouan, Tunis, 1982, cat. 388, p. 169.
} 
a Bari, datati tra X e XI secolo ${ }^{33}$, questo tipo di capitello non sembrerebbe altrimenti attestato.

Potrebbe essere inoltre seducente, ma assai improbabile, l'ipotesi di riconoscere nel capitello in questione una spoglia della basilica paleocristiana sottostante l'ottocentesco duomo di Pesaro, nella quale ci si sarebbe aspettata una qualche attestazione 'marmorea' di provenienza costantinopolitana, in considerazione del fatto che, all'indomani della riconquista bizantina, l'edificio venne estesamente restaurato con un intervento promosso da un personaggio di alto rango: il magister militum Giovanni, ricordato nell'iscrizione di un pannello del pavimento musivo, da riconoscersi nel valoroso generale al servizio di Giustiniano, più volte ricordato da Procopio, il quale si era distinto anche nel Piceno durante la guerra greco-gotica ${ }^{34}$.

Desta analoga perplessità l'assenza di manufatti marmorei 'd'importazione' nella chiesa metropolitana di Fermo, ugualmente oggetto di importanti restauri al termine della devastante guerra greco-gotica ${ }^{35}$.

Si ha comunque la netta impressione che dopo la metà del VI sec. il flusso dei marmi provenienti dall'area greco-costantinopolitana in ambito adriatico sia notevolmente scemato, se non addirittura cessato del tutto. Colpisce in effetti l'assenza di arredi marmorei anche nel caso nel vasto complesso di San Giusto, presso Lucera, in Puglia, la cui fondazione è certo legata ad una committenza elitaria, episcopale o aristocratica, riconducibile forse proprio al già ricordato magister militum Giovanni, dopo la riconquista dell'Apulia ${ }^{36}$.

Restano evidentemente aperti molti interrogativi sulla circolazione dei manufatti di marmo proconnesio in area adriatica nel corso della seconda metà del VI secolo e sotto tale prospettiva sarebbero necessarie ulteriori indagini, con un più meditato approfondimento sui contatti e sugli scambi e nel contempo sul ruolo svolto da Ravenna nel rifornimento di marmi finiti o non finiti per l'arredo architettonico e liturgico di quegli edifici in corso d'opera proprio intorno alla metà del VI secolo, come nel caso dei materiali del duomo e della chiesa di Santa Maria Formosa a Pola, della basilica eufrasiana di Parenzo e anche delle basiliche di Sant'Eufemia e di Santa Maria delle Grazie a Grado. Sarebbe altresì auspicabile un meditato riesame dei singoli casi, e non solo dei grandi centri, ma anche dell'ampia dispersione in area adriatica dei manufatti di stile costantinopolitano, con una aggiornata mappatura che ne dettagli le diverse tipologie, specificandone anche le condizioni di erraticità o di reimpiego. Andrebbe parallelamente approfondita anche l'indagine sulla circolazione di spolia marmorei approdati in gran numero nelle lagune venete in seguito alla conquista latina di Costantinopoli.

Stando alle tracce documentarie sinora censite in area marchigiana, emerge un panorama quanto mai eterogeneo, estremamente rarefatto, nel quale solo la città di Ancona sembra offrire un riscontro oggettivo per l'importazione dei materiali di marmo del Proconneso nella regione, favorito certo dal suo porto, approdo strategico nel medio Adriatico per le rotte commerciali. Ed è proprio sul volgere del V secolo che Ancona e il suo porto conobbero una rinnovata crescita di importanza, da ricollegare anche alla sua funzione di scalo e di presidio a controllo della vicina Osimo, sede metropolitana del Piceno e importante piazzaforte ostrogota ${ }^{37}$. All'importanza strategica del porto di Ancona fa cenno anche Procopio il quale ricorda come i 'romani', diventati padroni del mare e dopo essersi impossessati della città di Ancona, «potevano ammassare là tutte le provviste che facevano venire dalla Sicilia e dalla Calabria» (BG, II, 24).

Non ritengo peraltro azzardato ipotizzare che, accanto al più consistente gruppo di sculture riutilizzate nel San Ciriaco, ve ne fossero altri, forse di minore entità, destinati ad altre chiese di Ancona ${ }^{38}$. È infatti assai probabile che anche la basilica paleocristiana scoperta nel 1928 sotto la chiesa medievale di Santa Maria della Piazza, fosse provvista di elementi d'arredo marmorei di provenienza costantinopolitana. Malgrado l'esiguità del sopravvissuto, è possibile riconoscerne gli avanzi nei pochi frammenti marmorei trovati nello scavo dell'edificio, tra i quali, richiamo ancora una volta all'attenzione il frammento di un pilastrino semplicemente modanato con terminazione a bulbo (fig. 5a). Esso appartiene infatti ad una tra le classi di manufatti di tipo seriale più largamente esportati tra V e VI secolo, ben esemplificabile dai pilastrini riuniti (fig. 5b) nel lapidario della Rotonda di San Giorgio a Tessalonica. Ancor più interessante appare il frammento di un altro pilastrino, del tipo a colonnetta, del quale resta pure il piccolo capitello corinzio con quattro foglie d'acanto ${ }^{39}$ (fig. 6a), verosimilmente fun-

\footnotetext{
33 Cfr. M. DENNERT, op.cit.(n. 30), cat. 91-92, p. 45-46, tav. 16.

${ }^{34}$ Con ogni probabilità l'evergetismo del magister militum era suggellato in forma ufficiale dal monogramma, sciolto con il nome di Narsete, campito in un altro pannello dello stesso pavimento: R. FARIOLI CAMPANATI, La datazione del mosaico pavimentale della seconda fase della cattedrale di Pesaro. L'identificazione del committente. in Atti del III Colloquio AISCOM (Bordighera, 6-1o dicembre 1995), Bordighera, 1996, p. 457-466; EAD., I mosaici pavimentali della seconda fase della Cattedrale di Pesaro, in Picus 18, 1998, p. 7-29; EAD., I mosaici pavimentali di età giustinianea della cattedrale di Pesaro: partizione compositiva e significato degli spazi ecclesiali, in Atti dell'XI Colloquio AISCOM (Ancona, 16-19 febbraio 2005), Tivoli, 2006, p. 563-578, fig. 2, 5-7; L. RICCARDI, Il Piceno nel racconto di Procopio durante le guerre gotiche, in C. Barsanti, A. Paribeni, S. Pedone (eds.), Rex Theodericus. Il Medaglione d'oro di Morro d'Alba, Roma, 2008, p. 55-65.

${ }^{35}$ Sono stati appunto datati al terzo quarto del VI sec. i lacerti superstiti della pavimentazione musiva della chiesa paleocristiana ritrovata nel 1934-36 nel sottosuolo del duomo medievale: R. FARIOLI, Tangenze ravennati nell'arte musiva pavimentale paleocristiana del litorale medio-adriatico, in CARB 22 , 1975, p. 202-212, fig. 1-4; F. COCCHINI, La basilica paleocristiana di Fermo, in Atti VI CNAC...op.cit. (n. 22), p. 443-455.

${ }^{36} \mathrm{Il}$ coinvolgimento di questo personaggio verrebbe suggerito dal ritrovamento di un mattone siglato da un monogramma sciolto appunto con il nome Iohannis: G. VOLPE, Il mattone di Iohannis (San Giusto, Lucera, Puglia) in J.-M. Carrié, R. Lizzi Testa (eds.), Humana sapit. Etudes d’Antiquité Tardive offertes à Lellia Cracco Ruggini, Turnhout, 2002, p. 79-93.

${ }_{37}$ Cfr E. ZANINI, Le Italie bizantine. Territorio, insediamenti ed economia nella provincia bizantina d'Italia (VI-VIII) secolo, Bari, 1998, p. 150-15, Y.A. MARANO, op.cit. (n. 25), p. 166-167.

${ }^{38}$ Non sono emersi elementi marmorei di arredo dallo scavo del grande edificio ecclesiale di VI secolo situato in via Menicucci, cfr. M.C. PROFUMO, Topografia paleocristiana di Ancona: breve nota, in Actes XIe CIAC, Lyon, Vienne, Grenoble, Genève et Aoste (21-28 septembre 1986), Città del Vaticano, 1989, p. $290-297$.

39 L. SERRA, Restauri e scoperte in Santa Maria della Piazza di Ancona, in Bollettino d'Arte s. II, 9, 1929, p. 97-121, in part. p. 103, fig. 12 e 28; ID., L'arte nelle Marche, Pesaro, 1929, p. 31, fig. 48-55, 59; C. BARSANTI, Mosaici...op.cit. (n. 25), p. 190-191; EAD., Un inedito pluteo... op.cit. (n. 5), p. 24-25; Y.A. MARANO, op.cit. (n. 25), p. 166, fig. 5 .
} 


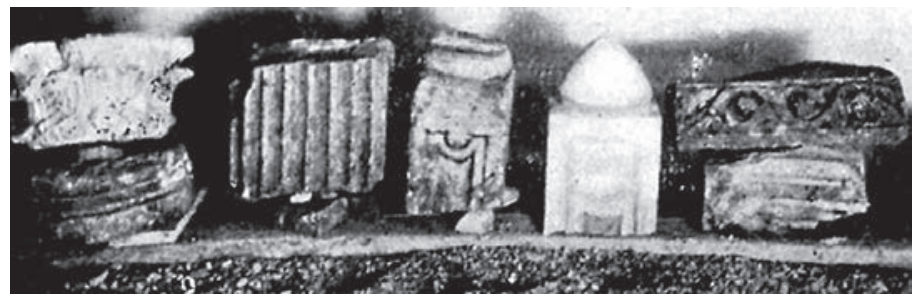

Fig. 5a. Ancona: frammenti dallo scavo della basilica paleocristiana sotto Santa Maria della Piazza (da Serra).

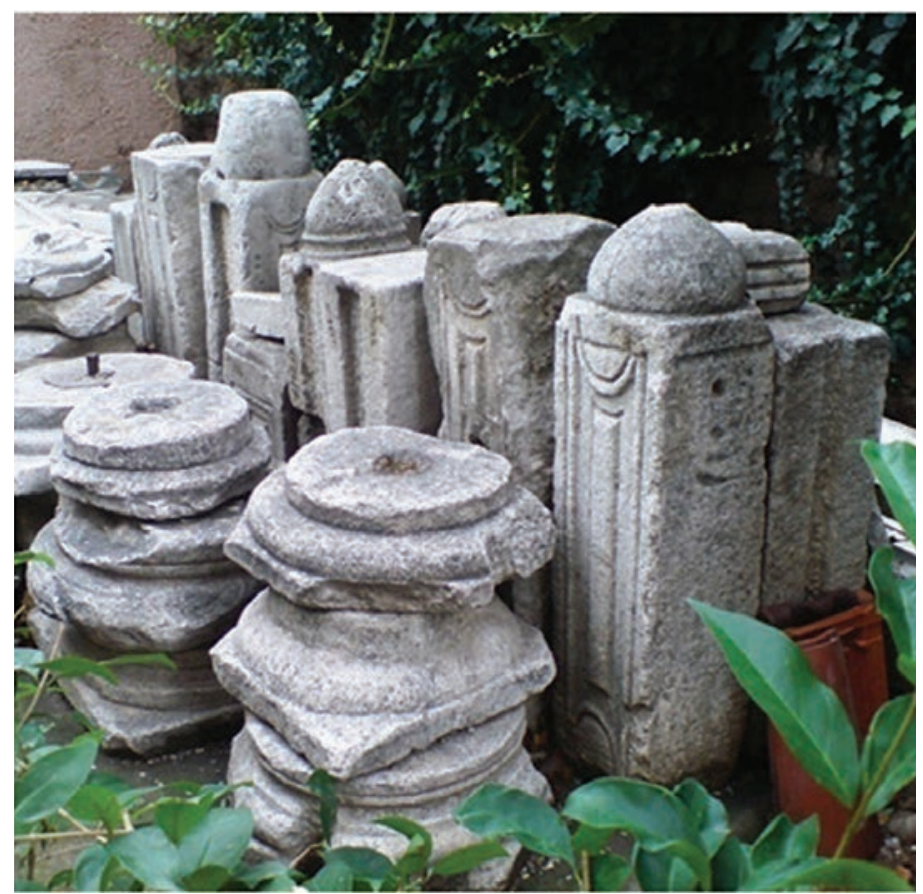

Fig. 5b. Tessalonica, lapidario della Rotonda di San Giorgio: pilastrini.

zionale ad un arredo liturgico. Anche in questo caso si tratta di un elemento di produzione standard ampiamente diffuso, il quale trova un considerevole numero di raffronti sia in ambito costantinopolitano ${ }^{40}$, sia in più distanti località ${ }^{41}$, tra cui basterà ricordare gli esempi di Salamina di Cipro ${ }^{42}$ e di Parenzo 43 (fig. 6b), e, con un significativo, forse affatto casuale, riscontro tra i materiali del carico di elementi architettonici e liturgici prefabbricati, di marmo proconnesio e breccia verde di Tessaglia, recuperati nel ben noto relitto naufragato a Marzamemi, sulle coste orientali della Sicilia ${ }^{44}$.

Un certo numero di spoglie di quella stessa basilica paleocristiana vennero tra l'altro recuperate per comporre la singolare panoplia di materiali marmorei che caratterizza appunto il prospetto della facciata duecentesca di Santa Maria della Piazza ${ }^{45}$.

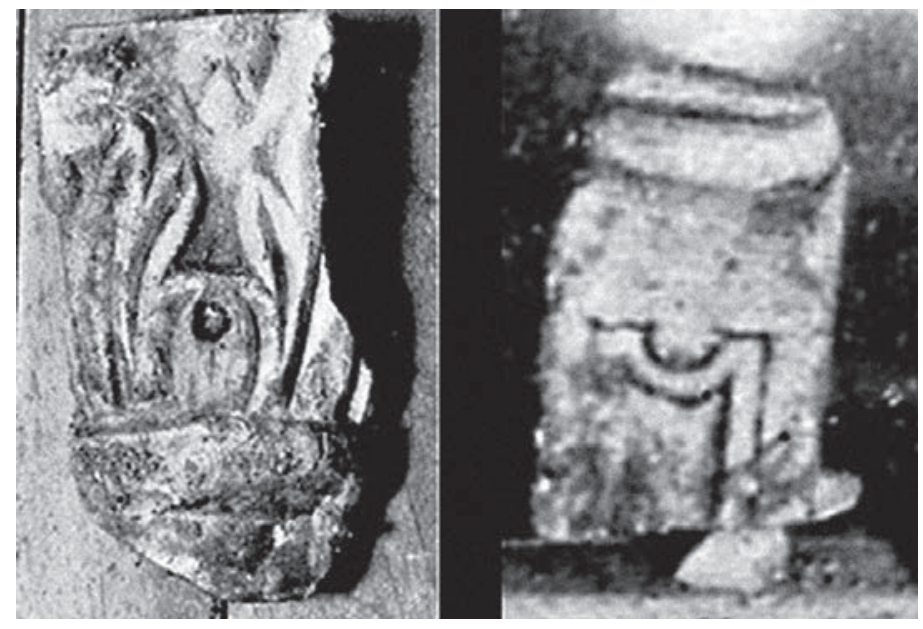

Fig. 6a. Ancona: frammento di pilastrino-colonnetta dallo scavo della basilica paleocristiana sotto Santa Maria della Piazza (da Serra).

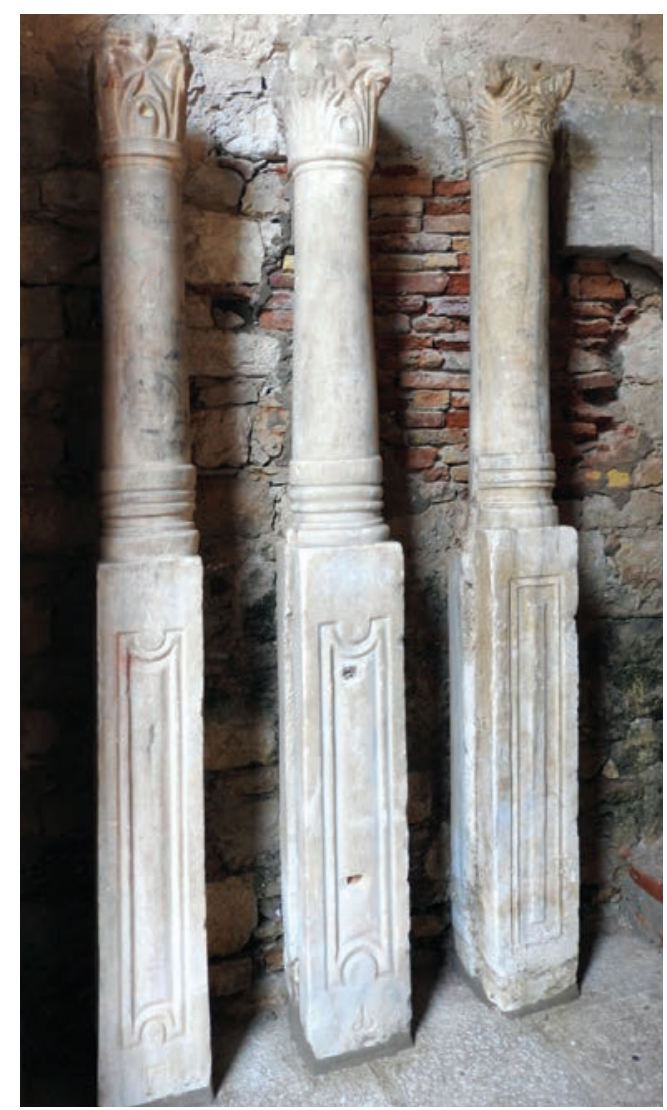

Fig. 6b. Parenzo, Basilica Eufrasiana: pilastrini-colonnetta.

Proveniva invece da un'altra chiesa anconetana di antica fondazione, dedicata al Salvatore, un pluteo di

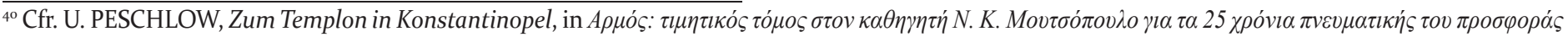

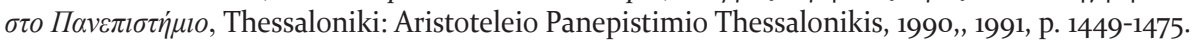

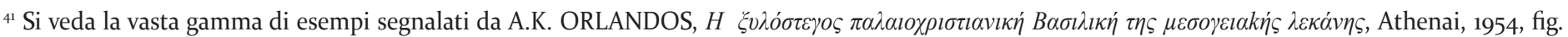
473-474, 490-492, 497; ed anche il cospicuo gruppo di pilastrini della basilica del vescovo Filippo a Stobi: I. NIKOLAJEVIĆ-STOIKOVIĆ, La décoration architecturale sculptée de l'époque bas-romaine en Macedoine, en Serbie et au Monténégro, Beograd, 1957, fig. 143; V.P. LILČIK, Corpus elementorum architecturae antiquae rei publicae Macedoniae, Pars secunda, Skopje, 2002, p. 830, fig. 9-10 e p. 1035.

${ }^{42}$ Cfr. G. ROUX, Salamine de Chypre XV, La basilique de la Campanopétra, Paris, 1998, p. 138-139, fig. 167-168.

${ }_{43}$ Cfr. E. RUSSO, Sculture del complesso eurasiano di Parenzo, Napoli, 1991, in particolare i n. 77, 97-97, p. 133-137, 155-156, fig. 103-104, 124-126; e, più recentemente, I. MATEJČIĆ, in I. Matejčić, S. Mustać (eds.), Il Patrimonio Artistico della Chiesa Istriana. Scultura dal IV al XIII secolo, Poreć, 2014, cat. 16, p. 88-9o, fig. 16,1-3.

${ }^{44}$ G. KAPITÄN, Elementi architettonici per una basilica dal relitto navale del VI secolo di Marzamemi (Siracusa), in CARB 27, 1980, p. 94-95, fig. 14-15; in proposito si veda anche. E.F. CASTAGNINO BERLINGHIERI, A. PARIBENI, Marble production... op.cit. (n. 2o). La datazione del relitto si collocherebbe nel primo quarto del VI secolo.

${ }^{45}$ Sull'argomento si rinvia al già citato convegno Il Piceno prima di Fiastra (cfr. supra, n. 22). 


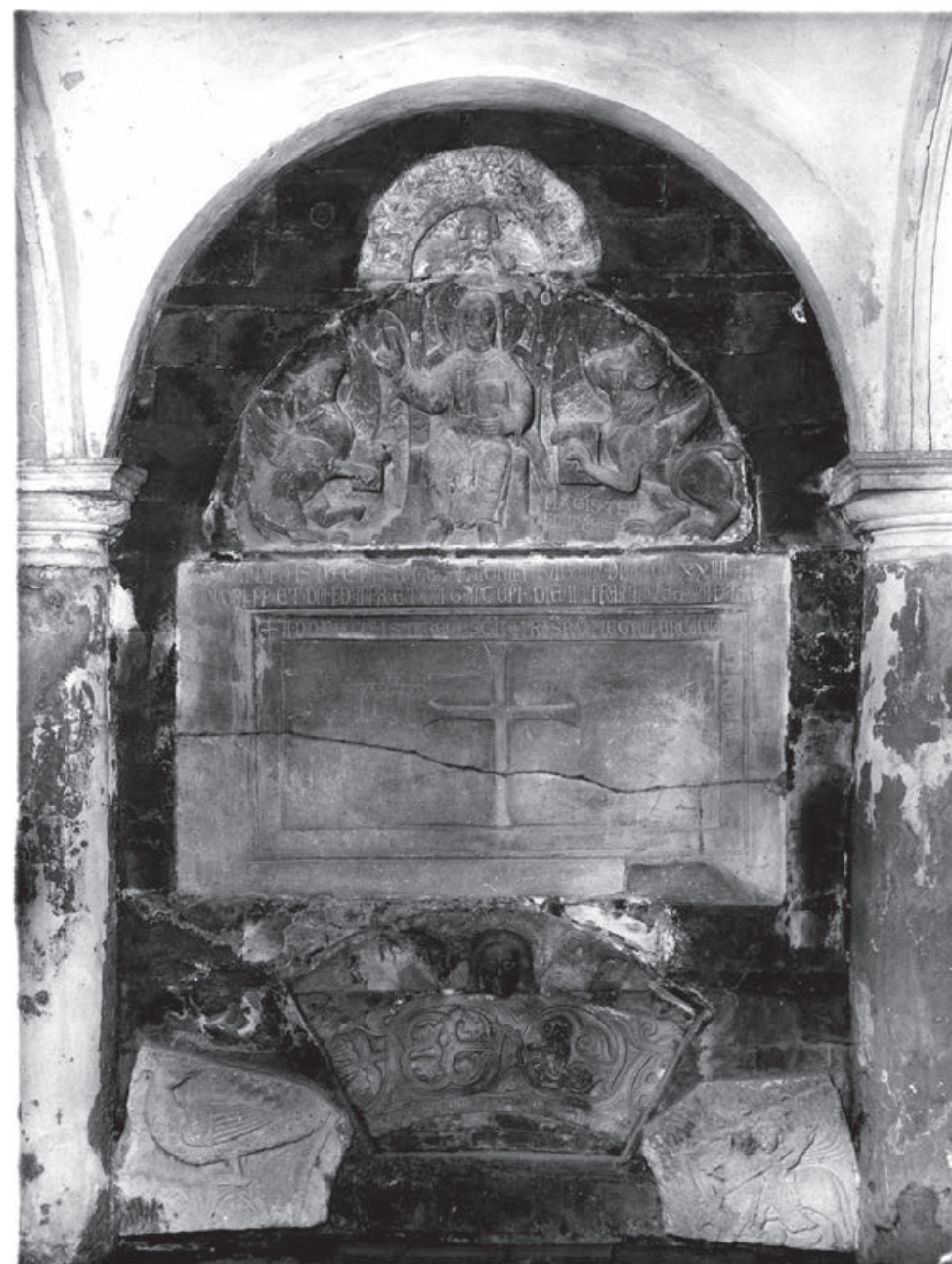

Fig. 7a. Ancona, San Ciriaco, lapidario allestito nella Cripta delle Lacrime: pluteo da San Salvatore (foto ICCD, fondo MPI 1927).

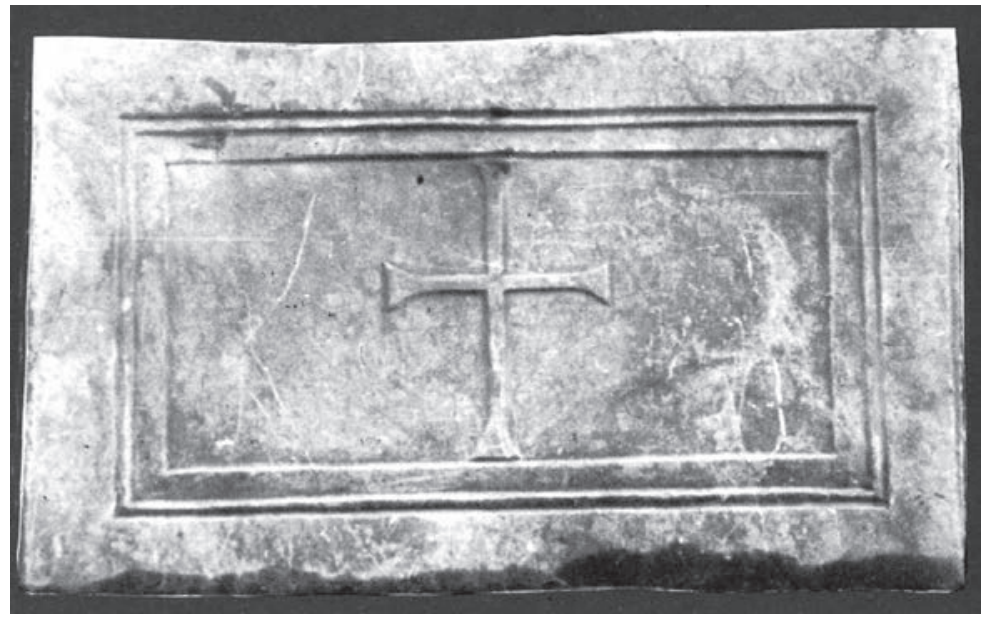

Fig. 7b. Tomi-Costanza, Museo Archeologico: pluteo (da Barnea). marmo proconnesio, decorato con un'elegante croce latina a estremità patenti (fig. 7a), vittima purtroppo dei bombardamenti dell'ultima guerra, assieme a gran parte dei materiali radunati nel lapidario che fin dal 1834 era stato allestito nella Cripta delle Lacrime (sottostante la Cappella del Crocifisso) del San Ciriaco. Di esso restano solo pochi lacerti dell'incorniciatura modanata, conservati nel deposito del Museo Diocesano, che recano incisa un'iscrizione dalla quale si apprende che nel 1224, la lastra sigillava il ricettacolo del sarcofago dei Santi Pellegrino e Fabiano ${ }^{46}$. L'origine costantinopolitana della lastra ${ }^{47}$, databile tra la fine del V e la prima metà del secolo seguente, trova piena conferma nella raffinata qualità del modellato della croce che campiva il liscio piano di fondo inquadrato da una incorniciatura modanata. Tra i molti confronti ricordo in particolare un pluteo di Tomi in Romania le cui caratteristiche formali denotano la medesima origine ${ }^{48}$ (fig. 7 b).

Ben più consistente ed importante è il complesso dei marmi di spoglio riutilizzati nel San Ciriaco, anche nel paramento lapideo della facciata (fig. 8), che si mostra al pari di un vero e proprio palinsesto di materiali eterogenei. Si distinguono appunto svariati inserti, rappezzi e risarcimenti realizzati a più riprese fino a tempi relativamente recenti con lastre di marmo ed anche con lacerti scultorei, tra cui, alcuni rilievi medievali49. Sono per lo più di recupero anche gli elementi marmorei riutilizzati come stipiti e architravi nelle finestre e nei portali. Diversi segmenti di marmo vennero inoltre scolpiti ed abilmente messi in opera come cornici, fregi figurati, modanature, colonnine, nel contesto della fastosa decorazione policroma del monumentale protiro duecentesco, attribuito a maestranze di formazione campionese ${ }^{50}$. La maggioranza di questi materiali è di un marmo bianco-ceruleo o bianco tendente al grigio, talora uniforme, altre volte percorso da liste più scure nel quale si può riconoscere a pieno titolo il marmo proconnesio. Parte di questi marmi venne probabilmente recuperata dalla preesistente basilica paleocristiana, mentre altri provengono forse dalle rovine dei circostanti edifici romani.

È ora il momento di entrare all'interno del San Ciriaco per prendere finalmente in esame gli elementi in funzione architettonica - colonne, basi, capitelli e pulvini - in opera nell'edificio (fig. 9), assieme ad alcune colonne di granito grigio, il cui reimpiego, va osservato, venne realizzato con studiata progettualità, secondo un criterio selettivo che tenne conto del tipo dei materiali in rapporto alla loro nuova collocazione.

Verosimilmente, e al riguardo, come si è accennato in apertura, gli studiosi sembrano concordi, queste sculture,

\footnotetext{
${ }_{4}^{46}$ La foto qui riprodotta risale al 1927 (Gabinetto Fotografico Nazionale - ICCD, fondo MPI 1927); la lastra è documentata anche da una ripresa fotografica realizzata tra il 1872 e il 1877 da J. H. Parker, (Raccolta Parker, Archivio DAI-ROM-PK.2675). Nell'immagine pubblicata da M. MARINELLI, op.cit. (n. 21), fig. 8, si distingue solo una porzione della lastra. Sulla storia del monumento e le sue spoglie, cfr. S. PIRANI, Considerazioni sui resti del sarcofago e sui monumenti epigrafici relativi ai SS. Pellegrino e Flaviano ad Ancona, in Atti VI CNAC... op.cit. (n. 22), p. 551-564, in part. p. 558-563, fig. 4-5; C. BARSANTI, Un inedito pluteo...op.cit. (n. 25), p. 24-25.

47. Esemplificativo al riguardo è il raffronto offerto da una lastra reimpiegata nel pavimento dell'esonartece della Santa Sofia: R. FLAMINIO, Gli elementi marmorei di reimpiego, in A. GUIGLIA GUIDOBALDI, C. BARSANTI, Santa Sofia...op.cit. (n. 28), p. 542-543, 605-610, con ampia rassegna di confronti.

${ }^{8}{ }^{8}$ Cfr. I. BARNEA, Les monuments paléochrétiens de Roumanie, Citta del Vaticano, 1977, p. 203, fig. 71,2.

${ }^{49}$ Ne fanno breve menzione C. POSTI, Il Duomo di Ancona, Jesi, 1912, p. 22; L. PANI ERMINI, La chiesa di San Lorenzo, in San Ciriaco... op.cit. (n. 21), p. 94-115, p. 108, fig. 26, 30; F. REDI, La basilica di San Ciriaco nel medioevo, ibidem (n. 21), fig. 33.

$5^{50}$ B. TEODORI, Il protiro della cattedrale di San Ciriaco ad Ancona: considerazioni dopo il restauro su un affascinante problema di scultura monumentale duecentesca, in San Ciriaco...op.cit. (n. 21), p. 189-227. Sono ugualmente di spoglio le colonne che sorreggono il protiro del portale laterale, come pure gli stipiti e l'architrave che ne incorniciano il varco: F. REDI, op.cit. (n. 49), p. 148, fig. 69.
} 


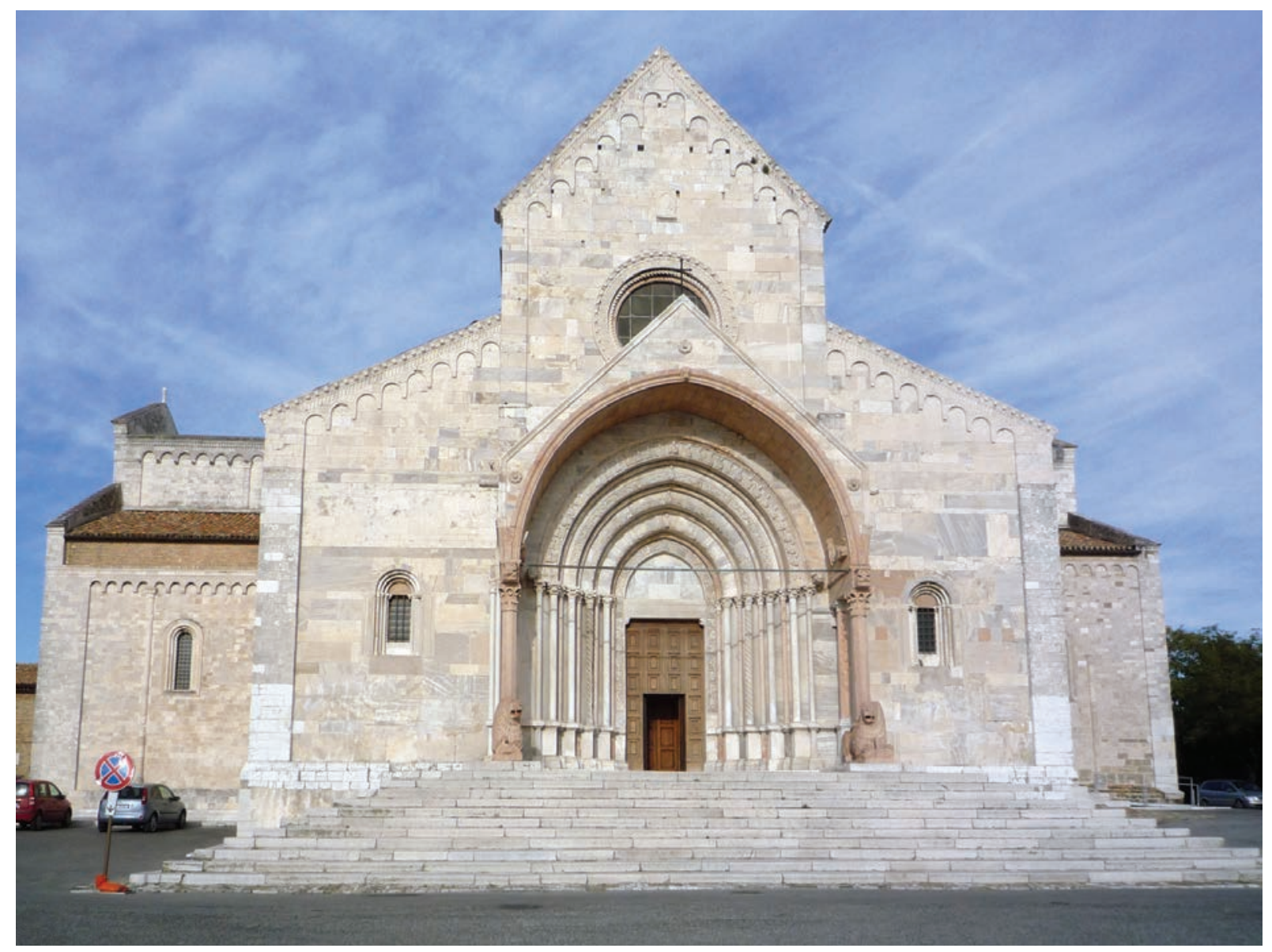

Fig. 8. Ancona, San Ciriaco: facciata.

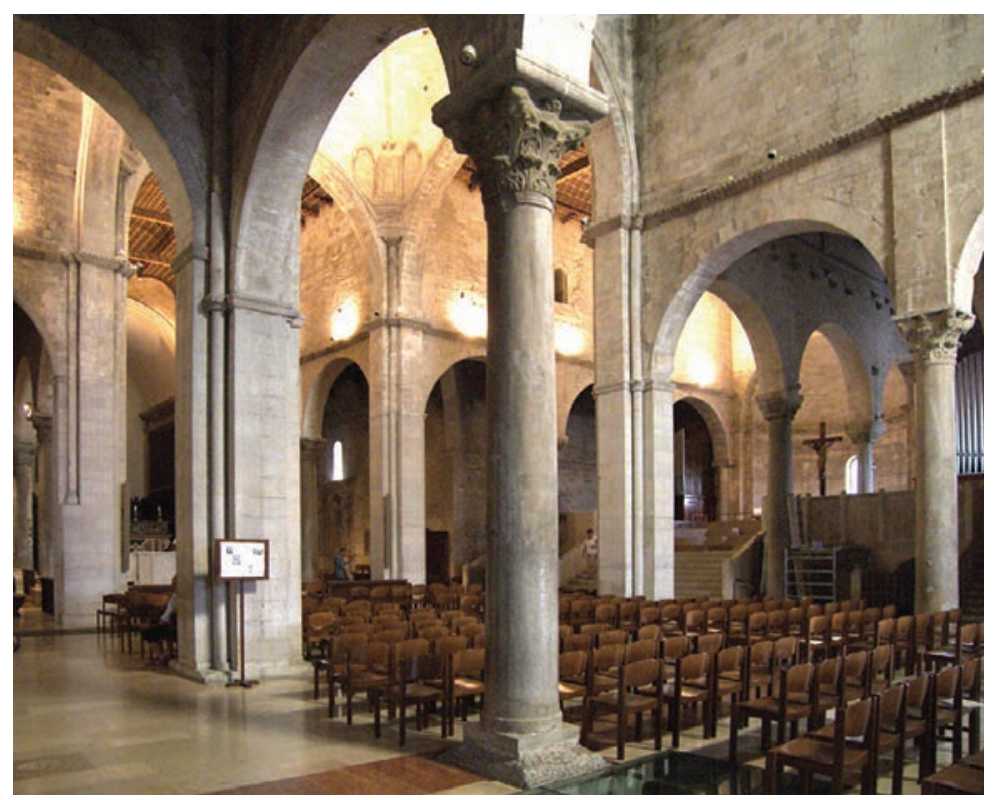

Fig. 9. Ancona, San Ciriaco: interno.

accomunate nel medesimo orizzonte formale e cronologico, facevano parte dell'arredo della basilica paleocristiana dedicata a San Lorenzo, un edificio di non grandi dimensioni, ma particolarmente sontuoso, provvisto anche di rivestimenti marmorei parietali e pavimentali, come lascerebbero supporre i pochi lacerti messi in luce nel sottosuolo del San Ciriaco durante le indagini archeologiche condotte all'indomani dell'ultima guerra ${ }^{51}$.

La fondazione del San Lorenzo, stando ad una tradizione - riferita comunque da tardi cronisti locali - sarebbe legata al nome di Galla Placidia ${ }^{52}$ e se pure avvolta in un alone di leggenda, tale tradizione potrebbe avere un sottofondo di verità, adombrando una committenza di alto rango e una munifica benevolenza che probabilmente si era fatta carico del dispendioso arredo marmoreo ${ }^{53}$. Del resto, come altrove, i marmi del Proconneso siglano sovente prestigiose fondazioni legate direttamente o indirettamente a importanti imprese di committenza ufficiale e, in alcuni casi, anche all'evergetismo imperiale ${ }^{54}$.

In passato, la costruzione del San Lorenzo è stata talora attribuita anche al vescovo Marcellino che resse la chiesa di Ancona durante gli ultimi anni della guerra greco-gotica, ma questa ipotesi, lievitata nella tradizione locale, senza alcun concreto riscontro documentario, contrasterebbe con la cronologia delle sue sculture, il cui arco produttivo si colloca tra la seconda metà del V sec. e i primi anni del secolo seguente, come si ricava appunto dai contesti archeologici dei materiali di raffronto, siano essi costantinopolitani, siano essi piuttosto materiali esportati in altre più distanti

\footnotetext{
${ }^{51}$ Cfr. F. REDI, op.cit. (n. 49), fig. 53 e 58.

${ }^{52}$ Cfr. L. PANI ERMINI, op.cit. (n. 49), p. 111-112.

${ }_{53}$ Sul coinvolgimento di Galla Placidia, cui fanno cenno gli storici locali: V. PIRANI, Quando la leggenda diventa storia, in G. MARIUZZI, V. PIRANI, C. LAUSDEI, San Ciriaco. Ricognizione canonica, storica e scientifica delle spoglie del patrono di Ancona, Ancona, 1986, p. 55-59; C. BARSANTI, Testimonianze...op.cit (n. 22), p. 404.

${ }^{54}$ Cfr. C. BARSANTI, L'esportazione...op.cit. (n. 23), p. 92-108.
} 


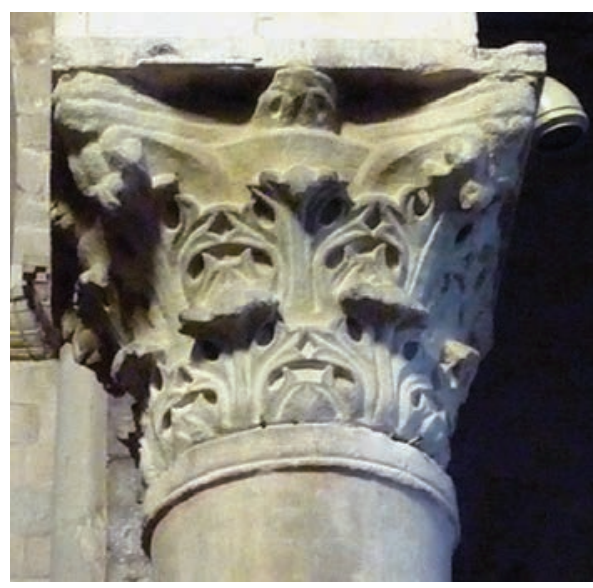

Fig. 10a. Ancona, San Ciriaco: capitello corinzio.

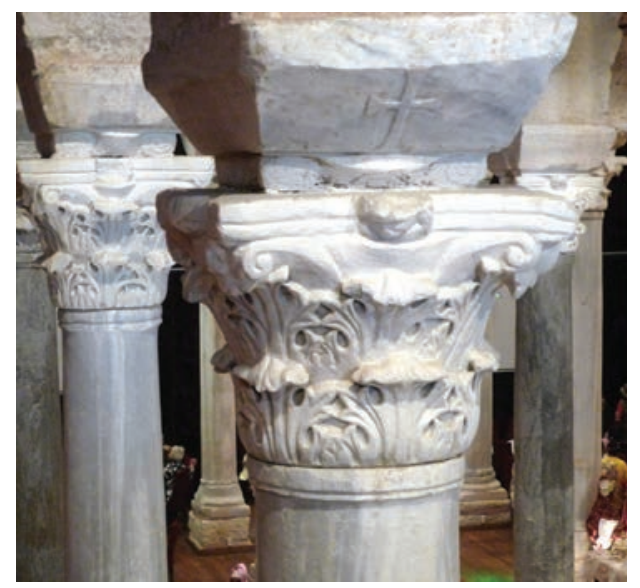

Fig. 10b. Istanbul, Cisterna detta della scuola (Sultan Sarnici): capitello corinzio.

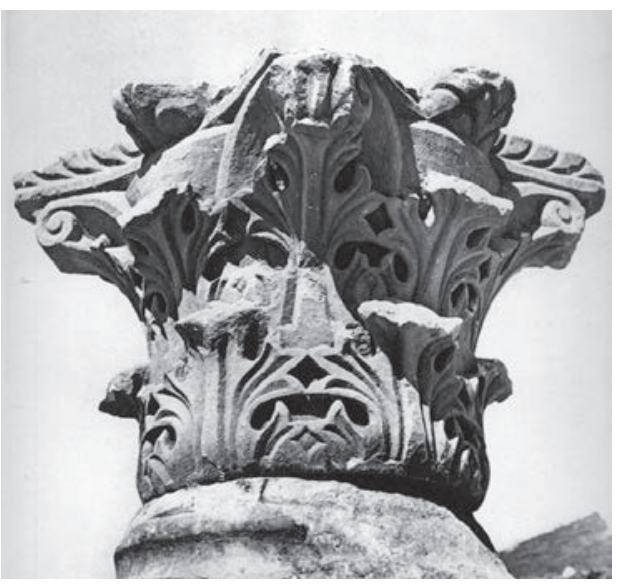

Fig. 10c. Filippi in Macedonia, basilica A: capitello corinzio (da Lemerle). regioni dell'Impero. E in favore di questa datazione, si è di recente espressa anche Letizia Pani Ermini, secondo la quale la fondazione del San Lorenzo potrebbe essere collegata al presidio fortificato sorto sull'acropoli anconitana al tempo di Teoderico, realizzato nel quadro del più ampio progetto di riorganizzazione delle difese del territorio della regione promosso e potenziato dal sovrano goto ${ }^{55}$.

Un primo gruppo di sculture comprende gli otto capitelli di tipo corinzio in opera nel braccio longitudinale dell'edificio su sette colonne di marmo proconnesio, di uguali dimensioni, mentre l'ottava, l'ultima a sinistra, è di granito grigio. Sulla fascia di base della prima colonna destra del braccio orientale è incisa la lettera greca ' $\theta$ ', identificabile come sigla di lavorazione ${ }^{56}$.

Simili tra loro per struttura e caratteri formali, gli otto capitelli corinzi (fig. 10a) mostrano solo esigue varianti nel modellato delle due corone che avvolgono il kalathos con sei foglie d'acanto, quella inferiore, e otto quella superiore, e i cui lobi, ricongiungendosi, creano zone d'ombra che danno forma alla così detta 'maschera d'acanto', uno stilema qualificante usualmente l'acanto bizantino ${ }^{57}$. Dalla corona superiore fuoriescono le volute a nastro, piuttosto sottili e a sezione quasi triangolare, desinenti in spirale sotto gli angoli dell'abaco semplicemente profilato; tra le volute emerge l'orlo semilunato del kalathos. Tali caratteristiche consentono di assimilare questo gruppo di capitelli al 'tipo 4' della ormai storica classificazione dei capitelli corinzi costantinopolitani a suo tempo proposta dal Kautzsch ${ }^{58}$.

Nell'ampio panorama documentario nel quale s'inseriscono i capitelli in questione, richiamo innanzi tutto all'attenzione quei confronti costantinopolitani, come gli esemplari riutilizzati nella Kalenderhane Camii59 e nella cisterna n. 9, la così detta Cisterna della scuola (fig. 1ob), che appaiono strettamente vicini, pressoché identici, ai capitelli del San Ciriaco $^{60}$. Allontanandoci dalla capitale, ricordo invece gli esemplari della basilica A di Amphipolis e quelli della basilica A di Filippi (fig. 10c), tutti datati intorno ai primi anni del VI secolo ${ }^{61}$, che mostrano ugualmente forti analogie formali con la serie anconetana.

Allo stato attuale, questa categoria di capitelli sembrerebbe assente nel panorama adriatico ${ }^{62}$ dove non mancano invece all'appello altre tipologie corinzie create dagli opifici del Proconneso. Numerosi sono soprattutto i capitelli a 'V' o ‘a lira', forse la categoria più largamente esportata dall'area costantinopolitana ${ }^{63}$. Sono attestati, ma in numero sensibilmente ridotto, anche i capitelli del tipo cosiddetto 'a medaglione' ${ }^{\prime 64} \mathrm{e}$

55 L. PANI ERMINI, op.cit. (n. 49), p. 99. A quest'ambito cronologico riconducono anche i pochi lacerti del mosaico pavimentale della primitiva basilica: C. BARSANTI, Mosaici...op.cit. (n. 25) 1995, p. 184; L. PANI ERMINI, op.cit. (n. 49), p. 104-107, fig. 19-21, 23.

${ }^{56}$ In proposito cfr. A. PARIBENI, Le sigle dei marmorari e l'organizzazione del cantiere, in A. Guiglia, C. Barsanti (eds.), Santa Sofia... op.cit. (n. 28 ), p. $651^{-}$ 734. Questa colonna e le altre del braccio orientale poggiano su piedistalli di muratura, tre di esse sono provviste di basi classiche di tipo attico, mentre la base della colonna di granito è di fattura medievale.

${ }^{57}$ M. MARINELLI, op.cit. (n. 21), p. 51-52, fig. 7, 6; V. FORTUNATI, Ancona/ San Ciriaco, Bologna, 1966, fig. p. 16; C. BARSANTI, Testimonianze...op.cit. (n. 22), p. 391-393, fig. 1; M. POLVERARI, Ancona e Bisanzio, Ancona, 1993, fig. 11.3; L. PANI ERMINI, La chiesa di S. Lorenzo, op.cit. (n. 49), p. 103, fig. 15.

$5^{8}$ R. KAUTZSCH, Kapitellstudien. Beiträge zu einer Geschichte des spätantiken Kapitells im Osten von vierten bis ins siebente Jahrhundert, Berlin-Leipzig, 1936, p. 53-6o.

59 U. PESCHLOW, Architectural Sculpture, in C. Striker, Y.D. Kuban (eds.), Kalenderhane in Istanbul. The Building, their History, Architecture and Decoration, Mainz, 1997, p. 102-103, tav. 76-80, con datazione all'ultimo quarto del V secolo.

${ }^{60}$ C. BARSANTI, Le cisterne di Istanbul: nuovi dati sulla scultura dal V al VII secolo. La cisterna n. 9 (la c.d. Cisterna della Scuola), in Acta XV CIAC, Toleti 2008, Città del Vaticano, 2013, p. 1481-1496, in part. p. 1491, fig. 1 e 9. Sempre in ambito costantinopolitano si veda anche un esemplare (inv. 129) nel lapidario della Santa Sofia e un altro (inv. 3252) nel giardino del Museo Archeologico (T. ZOLLT, Kapitellplastik Konstantinopels vom 4. bis 6. Jahrhundert $n$. Chr., Bonn, 1994, n. 426 e 440, p. 157, 161).

${ }^{61}$ Per Amphipolis, cfr. D. PALLAS, Les monuments paléochrétiens de Grèce découverts de 1959 à 1973, Città del Vaticano, 1977, p. 93, fig. 58; A. TADDEI, I monumenti bizantini dell'acropoli di Amphipolis, in Annuario SAIA 86, 2008, p. 253-310, in part. p. 270 e note 167-168, fig. 6; per Filippi, cfr. P. LEMERLE, Philippes et la Macédoine orientale à l'époque chrétienne et byzantine (BEFAR, 158), Paris, 1945, p. 405-407, tav. XI.

${ }^{62}$ Senza tener evidentemente conto degli esemplari reimpiegati nella basilica di San Marco, spoglie costantinopolitane giunte a Venezia dopo il sacco latino del 1204: F.W. DEICHMANN, Corpus der Kapitelle der Kirche von San Marco zu Venedig, unter Mitarbeit von J. Kramer und U. Peschlow, Wiesbaden, 1981, in part. cat. 11, p. 31, tav. 2.

${ }_{63}$ Oltre agli esemplari di Ravenna e del suo territorio, la diffusione di questo tipo di capitello è documentata a Durazzo e in area pugliese.

${ }^{64}$ Capitelli a medaglione sono documentati a Manastirine, a Ravenna e nel suo territorio (Pomposa e pieve Cesato, vicino Faenza),e, ad Ancona (cfr. infra). Per questa categoria di capitelli la cui produzione, riconducibile agli opifici del Proconneso, avviata nel corso della seconda metà del V si protrasse sino a tutta la prima metà del secolo seguente, si veda da ultima C. BARSANTI, Marmi costantinopolitani a Cipro, in M. Gianandrea, F. Gangemi, C. Costantini (eds.), 


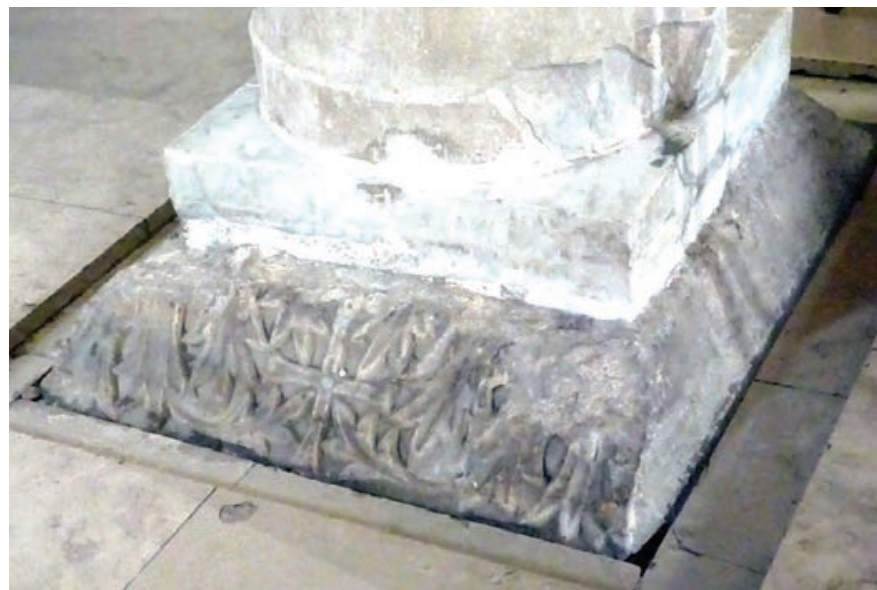

Fig. 11a. Ancona, San Ciriaco: pulvino reimpiegato come base.

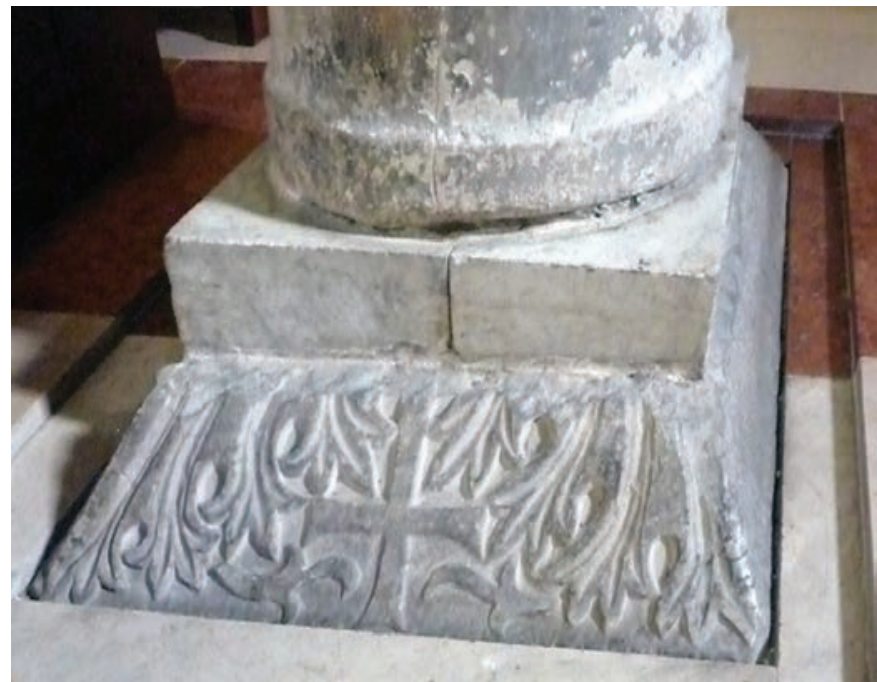

Fig. 11b. Ancona, San Ciriaco: pulvino reimpiegato come base.

quelli del 'tipo $7^{\prime 65}$, nonché il più raro 'tipo 8', testimoniato da un isolato esemplare nel Museo Archeologico di Durazzo. ${ }^{66}$

Ai capitelli corinzi del San Ciriaco erano quasi certamente coordinati dei pulvini. Un secondo gruppo di sculture comprende infatti tre imposte, o pulvini, tronco piramidali, riutilizzati capovolti come basi di tre colonne del braccio occidentale (d'ingresso) (fig. 11a-b) ${ }^{67}$.

Sulla fronte di tutte e tre le imposte è scolpito a bassorilievo il simbolico motivo della croce tra morbide foglie di acanto, una composizione che conosce un rilevante numero

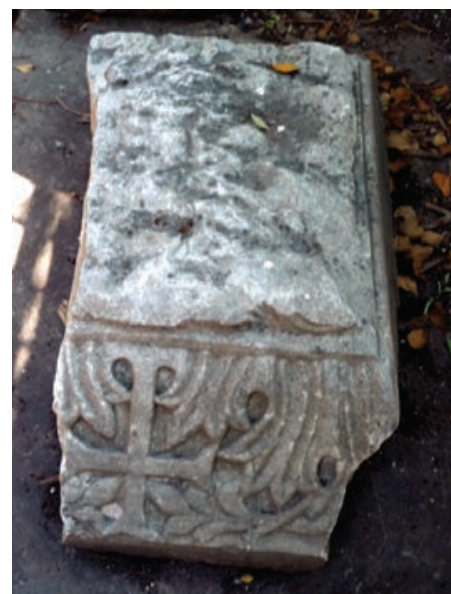

Fig. 12a. Istanbul, basilica di san Giovanni di Studio (Imrahor Camii): imposta.

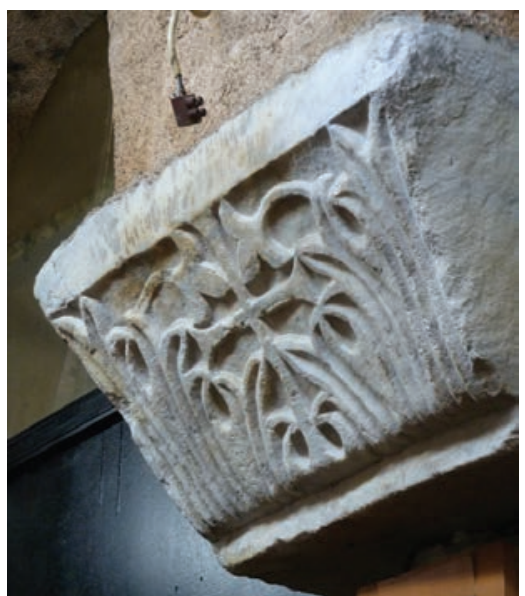

Fig. 12b. Istanbul, Rotonda del Myrelaion: imposta.

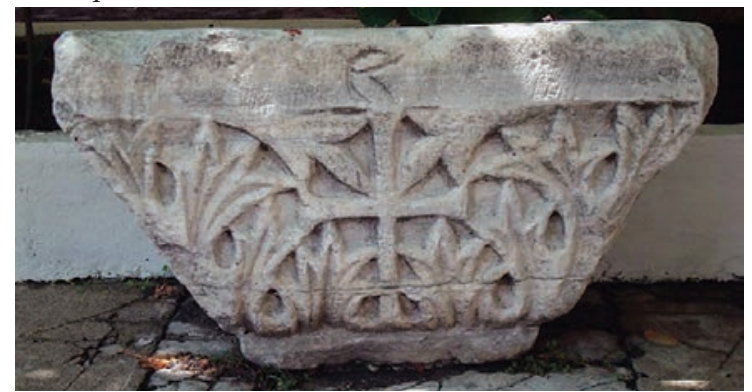

Fig. 12c. Istanbul, chiesa di Aya Kiryaki: imposta (da Coroneo).

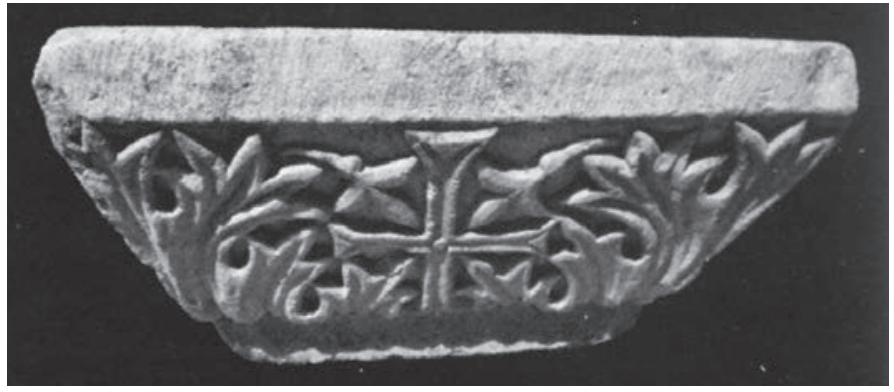

Fig. 12d. Silifke, Museo Archeologico: imposta (da Aydin).

di raffronti e i cui referenti cronologici sono individuabili nei capitelli e nelle imposte realizzate intorno alla metà del V secolo per la basilica costantinopolitana del San Giovanni di Studio $^{68}$ (fig. 12a). Particolarmente significativo per il tema decorativo in questione, diffuso in svariatissime varianti nel

Il potere dell'arte nel Medioevo. Scritti in onore di Mario D'Onofrio, Roma, 2014, p. 261-272; EAD., Capitelli corinzi del tipo cosiddetto a medaglione con inserti decorativi, in Atti del convegno Decor. Decorazione architettonica nel mondo romano, Sapienza Università di Roma, 21-24 maggio 2014, in.corso di stampa. ${ }^{65}$ Questo tipo di capitello è testimoniato da un numero ridotto di esempi: tre a Dubrovnik (B. ŠILJEG, Early Christian Marble Corinthian Capitals from Dubrovnik, in Prilozi Instituta za arheologiju u Zagrebu 24, 2007, p. 257-261), e un isolato esemplare segnalato a Trieste - P. PENSABENE, La decorazione architettonica, l'impiego del marmo e l'importazione di manufatti orientali a Roma, in Italia e in Africa (II-VI sec. d.C.), in A. Giardina (ed.), Società Romana e Impero Tardoantico. Le merci e gl'insediamenti,. III, Bari, 1986, 352, fig. 20 - e dal più consistente gruppo di Durazzo, comprendente gli esemplari del Foro Circolare (P. PENSABENE, Inscribed Architectural Elements from the Prokonnesos in Durazzo, Tartous, Cilician Aphrodisias and Caesarea, in J.J. Hermann, N. Herz, R. Newman (eds.), Interdisciplinary Studies on Ancient Stone, Proceedings of the Fifth International Conference for the Study of Marble and Other Stones in Antiquity, Boston 1998, London, 2002, p. 328-334, in part. p. 330, fig. 7; P. PENSABENE, C. BARSANTI, op.cit. (n. 25), p. 471, fig. 18-19), e quelli del Museo Archeologico (A. HOTI, Plastikë arkitektonike dekorative paleokristiane nga qyteti i Durrësit, in Iliria 27, 1-2, 1997, p. 325-347, in part. n. 9-10, p. 329-330, tav. II,1-2), due dei capitelli recano incise sul piano d'imposta, l'uno le lettere I X K V, l'altro le lettere TP, identificabili come marchi di lavorazione; la sigla TP, ricordo, è incisa anche su un capitello costantinopolitano del medesimo tipo 7 ( C. BARSANTI, L'esportazione... op.cit. (n. 23), p. 116, fig. 23).

${ }^{66}$ A. HOTI, op.cit. (n. 65), p. 328, fig. 2. Al di fuori di Costantinopoli, oltre al capitello in questione, sono noti solo i due esemplari nella vicina Bitinia (Karakilise e Museo di Iznik) ed uno a Corinto, dalla basilica di Kodrato.

${ }^{67}$ C. BARSANTI, Testimonianze...op.cit. (n. 22), p. 393-395, fig. 3; M. POLVERARI, op.cit. (n. 57), fig. 11.4; L. PANI ERMINI, op.cit. (n. 49 ), p. 103 , fig. 18. Queste sculture sono state 'riscoperte' al momento del ripristino del livello pavimentale di XII sec. realizzato nel corso dei restauri condotti all'indomani dell'ultima guerra (1948-50). La quarta imposta, sotto la prima colonna destra è di fattura moderna.

${ }^{68}$ T. ZOLLT, op.cit. (n. 6o), n. 1-3, p. 9-10, tav. 1-2, fig. 25-26. Si vedano anche i capitelli di poco successivi della basilica di San Leonida a Corinto-Lechaion: C. BARSANTI, Testimonianze... op.cit. (n. 22), p, 395, fig. 4. 


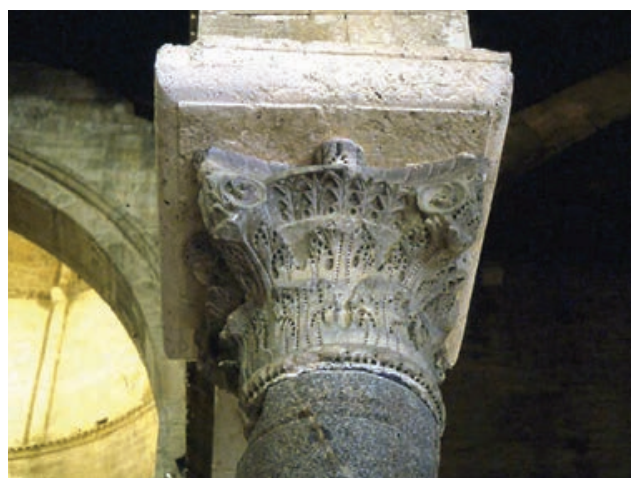

Fig. 13a. Ancona, San Ciriaco, cappella della Madonna: capitello composito con acanto finemente dentellato.

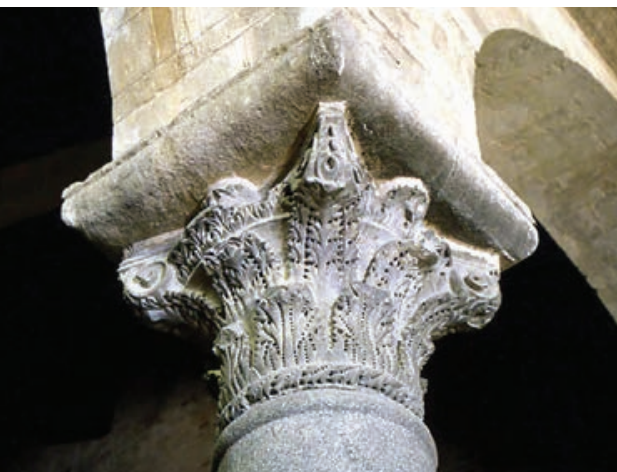

Fig. 13b. Ancona, San Ciriaco, cappella della Madonna: capitello composito con acanto finemente dentellato.

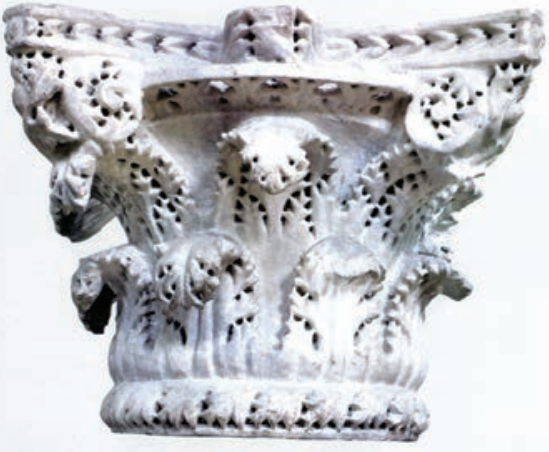

Fig. 13c. Cherson, Museo Archeologico: capitello composito con acanto finemente dentellato (da The Legacy of Byzantine Cherson). repertorio della scultura di V-VI secolo, è il confronto offerto da altri esempi in ambito costantinopolitano, tra i quali le imposte in opera nella cisterna nella rotonda del Myrelaion ${ }^{69}$ (fig. 12b), quelle nel lapidario dell'Ayasofya Müzesi ${ }^{70}$ e nel giardino del Museo Archeologico di Istanbul ${ }^{71}$, ed anche una meno nota imposta nel cortile della chiesa tardo ottocentesca di Aya Kiryaki nel quartiere di Kumkap ${ }^{72}$ (fig. 12c), alle quali si possono aggiungere altri più distanti esempi, come le imposte nel Museo di Silifke (fig. 12d), sicuramente importate dall'area costantinopolitana ${ }^{73}$; non sembrano invece trovare corrispondenze tra i pur numerosi esempi ravennati, per lo più di manifattura locale.

Un terzo gruppo di sculture è rappresentato dalla coppia di capitelli collocati sulle due colonne di granito situate ai lati dello scalone che conduce alla cappella della Madonna ${ }^{74}$ (fig. 13a-b). Entrambi di tipo composito con foglie di acanto finemente dentellato, elemento qualificante della categoria, sono tra loro differenti sia nella morfologia delle foglie di acanto, sia negli ornati sussidiari. Diverso è infatti il decoro dell'echino che, nel capitello a sinistra, reca un fregio di palmette a sette lobi appuntite ritagliato a giorno, mentre nell'altro, il capitello a destra, il fregio presenta fogliette a cinque lobi; in questo stesso capitello, le foglie d'acanto della corona superiore sono incorniciate da una delicata smerlatura fitomorfa.

Tra i numerosissimi capitelli di questa categoria che ebbe una straordinaria diffusione in tutto il bacino mediterraneo, nelle regioni pontiche ed anche in area adriatica ${ }^{75}$, si possono isolare svariati esemplari che mostrano un analogo arricchimento delle foglie della corona superiore, come quelli in opera nella basilica dell'Acheropoietos di Tessalonica, datati al terzo quarto del $\mathrm{V}$ secolo ${ }^{76}$, quelli della basilica scavata nel 1935 a Cherson in Crimea (fig. 13c) ${ }^{77}$ e come pure i due capitelli di ambito romano, riutilizzati, l'uno, nella chiesa di San Saba e, l'altro, nella chiesa di Santa Maria in Cosmedin ${ }^{78}$.

Il quarto gruppo di sculture riutilizzate nel San Ciriaco comprende infine i tre capitelli del tipo ionico ad imposta scampati, ma non senza guasti, dai bombardamenti dell'ultima guerra, in opera sulle colonne di marmo proconnesio della Cappella del Crocifisso: le due più grandi, situate all'esterno, ai lati dello scalone di accesso, e le due più piccole, all'interno $^{79}$. Il quarto capitello, in opera sulla prima colonna

\footnotetext{
${ }_{69}$ T. ZOLLT, op.cit. (n. 6o), n. 158-16o, tav. 32.
}

$7^{70}$ Inv. 169. I fianchi dell'imposta sono stati riscolpiti in epoca mediobizantina: T. ZOLLT, op.cit. (n. 6o), cat. 187, p. 78; A. GUIGLIA, C. BARSANTI, St. Sophia Museum Project 2006: The Marble Sculptures of the Middle-Byzantine Period, in 25. Araștırma Sonuçları Toplantısl 2. Cilt (Kocaeli, 28 Mayıs-1 Haziran 2007), Ankara, 2008, p. 117-132, in part. p. 121, fig. 4.

${ }^{71}$ Inv. 2308: G. MENDEL, Catalogue des sculptures grecques, romaines et byzantines, III, Constantinople, 1914, n. 1245, p. 469.

${ }_{72}$ La scultura è stata segnalata da R. CORONEO, Reimpiego di sculture costantinopolitane a Istanbul: un indagine sul campo, in ArcheoArte. Rivista elettronica di Archeologia e Arte Supplemento 2012 al numero 1, p. 605-627, in part. p. 614, fig. 20. Sulla fascia di bordo è incisa la sigla $€ Y$, che trova ampio riscontro in ambito costantinopolitano, cfr. A. PARIBENI, Le sigle dei marmorari... op.cit. (n. 56), p. 671, 731, fig. 375.

${ }^{73}$ Cfr. A. AYDIN, Erken Hiristiyanlık - Bizans Dönemi Mimari Elemanlarının, in S. Durugönül (ed.), Silifke Müzesi Taş Eserlerer Kataloğu. Heykeltırașlık ve Mimari Plastik Eserlerer, Mersin, 2013, cat. 341-345, pp. 279-281. Si vedano anche le imposte di Cherson in Crimea: A. BIERNACKI, The Early-Byzantine Architectural Elements and Details of Chersonesus Taurica, Poznan, 2009, n. 549/973 e 478/973, p. 52-53, fig. 30, tav. 102-103; The Legacy of Byzantine Cherson, Sevastopol-Austin, 2011, cat. 14, p. 430, fig. a p. 144.

${ }^{74}$ M. MARINELLI, op.cit. (n. 21), p. 52, fig. 7,5; V. FORTUNATI, op.cit. (n. 57), fig. a p. 16; C. BARSANTI, Testimonianze...op.cit. (n. 22), p. 395-397, fig. 5-6; M. POLVERARI, op.cit. (n. 57), fig. 11.5; L. PANI ERMINI, op.cit. (n. 49), p. 103, fig. 16; P. PENSABENE, C. BARSANTI, op.cit. (n. 25 ), p. 470, fig. 16.

75 Per le caratteristiche morfologiche di questa categoria di capitelli e la sua vasta diffusione cfr. C. BARSANTI. L'esportazione...op.cit. (n. 23), p. 142-146; J. KRAMER Bemerkungen zu den Methoden der Klassifizierung und Datierung frühchristlicher östromischer Kapitelle, in U. Peschlow, S. Möllers (eds.), Spätantike und byzantinische Bauskulptur. Beiträge eines Symposions in Mainz, Februar 1994, Stuttgart, 1998, p. 43-58; J.-P. SODINI, Deux chapiteaux byzantins découverts à l'abbaye de Valmagne (Hérault), in CRAI, 2003, p. 865-887.

${ }^{76}$ Cfr. J. KRAMER, op.cit.(n. 65), p. 56, tav. 13, 19.

77 A. PÜLZ, Die frühchristlichen Kirchen des Taurischen Chersonesos/Krim, in Mitteilungen zur christlichen Archäologie 4, 1998, p. 45-78, in part. p. 53, fig. 5; A. BIERNACKI, op.cit. (n. 73 ), cat. 773/973, 555/973, p. 40-42, tav. 62; L.G. KHRUSHKOVA, Chersonèse en Crimée aux IVe-Vle siècles: topographie et cronologie, in Acta XV CIAC.op.cit. (n. 41), p. 387-402, in part. 394, fig. 3; The Legacy of Byzantine Cherson...op.cit. (n. 73), cat. 12, p. 428, fig. a p. 148. Si veda anche la coppia nel chiostro di Santa Apollonia a Venezia: F.W. DEICHMANN, op.cit. (n. 62), cat. A 11 e A 13, p. 145, tav. 48.

${ }^{78}$ J. KRAMER, Spätantike korintische Säulenkapitelle in Rom, Wiesbaden, 1997, p. 148-149; C. BARSANTI, Capitelli di manifattura costantinopolitana a Roma, in F. Guidobaldi, A. Guiglia Guidobaldi (eds.), Ecclesiae Urbis, Atti del congresso internazionale di studi sulle chiese di Roma (IV-X secolo), Roma, 4-10 settembre 200o, Città del Vaticano, 2002, p. 1443-1478, in part. p. 1474, fig. 19; P. PENSABENE, Roma su Roma. Reimpiego architettonico, recupero dell'antico e trasformazione urbana tra il III e il XIII secolo, Città del Vaticano, 2015, cat. 952-953, p. 958, fig. 910-911.

${ }^{79}$ M. MARINELLI, op.cit. (n. 21), p. 52, fig. 7,2 e 4; V. FORTUNATI, op.cit. (n. 57), p. 11, fig. 2 a p. 16; C. BARSANTI, Testimonianze...op.cit. (n. 22), p. 397-401, fig. 7 e 9; L. PANI ERMINI, op.cit. (n. 49), p. 103, fig. 17. 


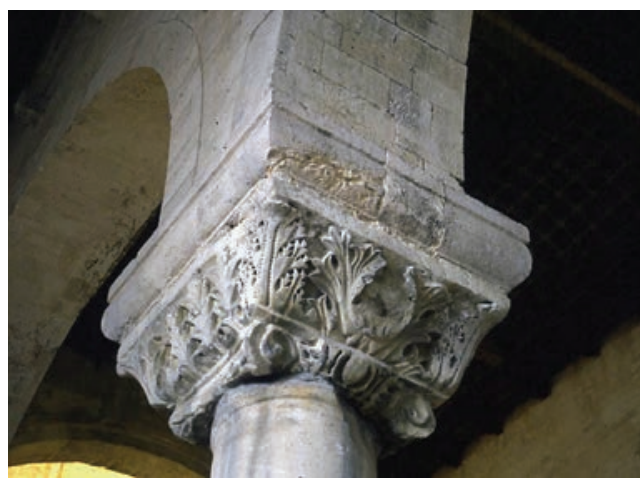

Fig. 14a. Ancona, San Ciriaco, cappella del Crocifisso: capitello ionico ad imposta.

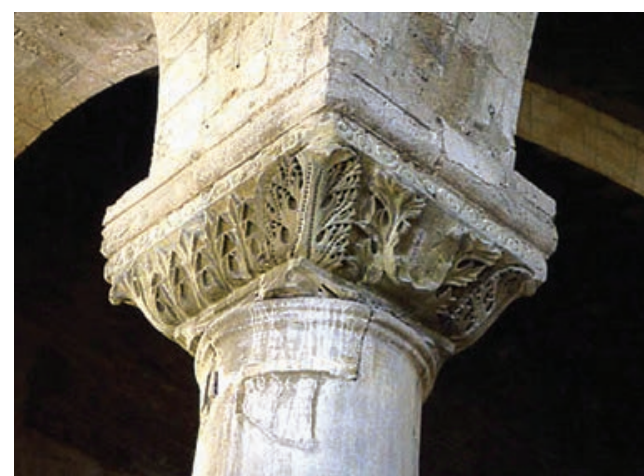

Fig. 14b. Ancona, San Ciriaco, cappella del Crocifisso: capitello ionico ad imposta.

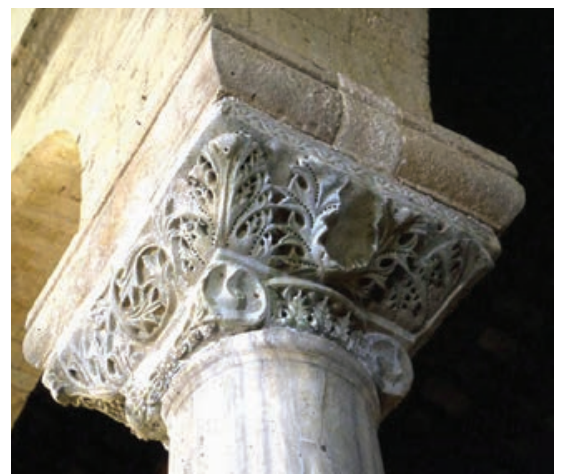

Fig. 14c. Ancona, San Ciriaco, cappella del Crocifisso: capitello ionico ad imposta. a destra della scalinata è invece di fattura medievale, che replica abbastanza fedelmente la forma e il decoro degli esemplari di V-VI secolo, in particolare quello del secondo capitello a destra, realizzato evidentemente per completare il "quartetto"80.

Se pure accomunati dalla medesima struttura decorativa, i tre capitelli si differenziano in alcuni particolari. Il secondo a destra (che chiameremo A) (fig. 14a) e il primo a sinistra (che chiameremo B) (fig. 14b), mutilo della zona ionica, sono grosso modo identici; mentre il secondo capitello a sinistra (che chiameremo C) (fig. 14c), appare diverso nelle proporzioni e anche nel lessico decorativo. Tutti e tre esibiscono sulla fronte, ugualmente rivolta verso l'abside, forse per celarne la frammentarietà, una composizione decorativa piuttosto fragile, ricorrente in questo particolare tipo di capitello: una sorta di cartoccio emergente dal piano di fondo, composto da foglie di acanto spinoso lavorate a giorno che in origine accoglievano forse una croce; altre due foglie di acanto, ma con lobi finemente dentellati, si dispongono sugli angoli dell'imposta; tra le volute della zona ionica, emerge dall'echino una serie di foglie ritagliate a giorno i cui bordi sono percorsi da sottili spine; esse si sovrappongono a quattro lisce foglie e sono sottese ad un sottile listello; il lato opposto presenta una composizione grosso modo analoga, priva però di lavorazione a giorno; tra le volute trova posto un ovolo nel guscio tra mezze palmette. Sul bordo superiore dell'imposta dei tre capitelli si svolge senza soluzione di continuità un sinuoso, esile tralcio vegetale stilizzato.

I lati dell'imposta del capitello $C$ recano uno stelo fogliato che si avvolge in spirale, mentre sui lati delle imposte dei capitelli A e B, caratterizzate peraltro da un certo slancio verticale, si dispongono paratatticamente, in posizione verticale, quattro palmette con nove lobi appuntiti, che sembrerebbero emergere dalla sottostante zona ionica.
Ad un esame più ravvicinato si ha l'impressione che il motivo del girale scolpito sul capitello $C$, caratterizzato peraltro da una fattura alquanto esitante, per non dire corsiva, possa essere il frutto di un restauro seriore, forse realizzato al momento del reimpiego, volto probabilmente ad eliminare quella sbarra liscia della quale si distingue una minima porzione appena sopra i balustri. Tale sbarra, emergente su entrambi i lati, che ricorre in molti capitelli di manifattura protobizantina, funzionale alla messa in opera di un telaio, forse di finestra, divideva in due parti il capitello, talora diversamente scolpite ${ }^{81}$, come appunto nel nostro caso e come mostrano, ad esempio, i capitelli di Nea Anchialos ${ }^{82}$, metà lavorati a giorno, evidentemente la parte rivolta verso l'interno dell'edificio, e metà con modellato pieno, che era invece rivolta verso l'esterno.

Anche questi capitelli rientrano nell'ambito di una categoria di formulazione costantinopolitana che riunisce le semplici imposte e i capitelli ionici ad imposta, in entrambi i casi viene coniugato, con molteplici varianti, il medesimo codice decorativo, per lo più caratterizzato dall'abbinamento dell'acanto spinoso e dell'acanto finemente dentellato. È una categoria forse meno diffusa delle precedenti, ma ugualmente testimoniata da un gran numero di esemplari esportati dagli opifici della capitale tra la seconda metà del V e i primi decenni del VI secolo che hanno come significativo referente cronologico, l'imposta dalla basilica di San Giovanni di Studio, oggi nei Musei di Berlino, datata, ricordo, intorno alla metà del $\mathrm{V} \mathrm{secolo}{ }^{83}$, alla quale possiamo avvicinare anche le tre imposte di Durazzo, di cui una giace erratica, nel Foro circolare (fig. 15a), mentre le altre due sono conservate nel locale Museo Archeologico ${ }^{84}$.

Per quanto riguarda invece la classe dei capitelli, documentati in ambito costantinopolitano dalla coppia di esemplari reimpiegati nella chiesa nord del Monastero

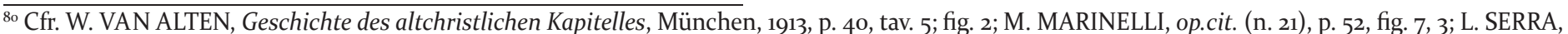
Restauri e scoperte, op.cit. (n. 39), p. 95, fig. 164, 166; V. FORTUNATI, op.cit. (n. 57), p. 11, fig. 2 a p. 13; C. BARSANTI, Testimonianze...op.cit. (n. 22 ), p. 399. ${ }^{81}$ In proposito, cfr. J.-P. SODINI, Un chapiteau «mixte» d'époque paléochrétienne à Delphes, in Rayonnement grec. Hommages à Charles Delvoye, Bruxelles, 1982 , p. 325-340.

${ }^{82}$ Cfr. V. VEMI, Les chapiteaux ioniques à imposte de Grèce à l'époque paléochrétienne (BCH Suppl. XVII), Paris, 1989, n. 100, 112, p. 123-124, 127-128, tav. 35, 37. ${ }^{8}$ Cfr. T. ZOLLT, op.cit. (n. 6o), p. 245-246; G. MIETKE in L. Wamser (ed.), Die Welt von Byzanz-Europas östliches Erbe - Glanz, Krisen und Fortleben einer tausendjährigen Kultur, München, 2004, cat. 85, p. 68; A. GUIGLIA, C. BARSANTI, S. PEDONE, St. Sophia Museum Project 2007: The Marble Sculptures of St. John Studius (Imrahor Camii), in 26. Araștırma Sonuçları Toplantısı, 3.Cilt (Ankara, 26-30 Mayis 2008), Ankara, 2009, p. 315, fig. 2a-b. All'imposta di Berlino si possono accostare i due esemplari in opera nella cisterna costantinopolitana n. 9, detta 'della scuola' (C. BARSANTI, Le cisterne...op.cit. (n. 6o), p. 1493, fig. 9), e, sempre a Costantinopoli, le due imposte reimpiegate nella chiesa nord del Monastero di Costantino Lips (T. ZOLLT, op.cit. (n. 6o), cat. 129-140, p. 61-62, tav. 29).

${ }^{84}$ Cfr. A. HOTI, op.cit. (n. 65), n. 16, p. 331, tav. IV,1; n. 18, p. 332, fig. 5. Si veda anche un'imposta nel Museo di Silifke: A. AYDIN, op.cit. (n. 73), cat. 349350, p. 283.
} 


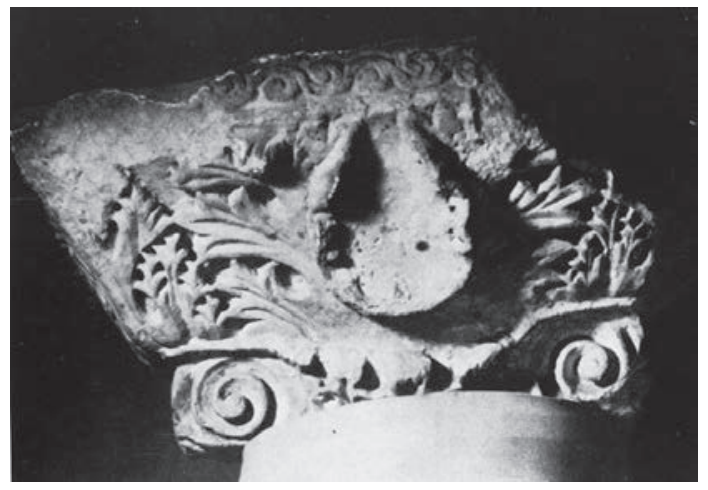

Fig. 15a. Mosca, Museo Storico: capitello ionico ad imposta da Cherson (da Bortoli Kazanski).

di Costantino Lips, nonché, ma in modo indiretto, dagli esemplari in opera nel parekklesion della basilica di San Marco a Venezia ${ }^{85}$, il confronto più stringente per i capitelli anconetani è piuttosto offerto dagli esemplari di Cherson in Crimea $^{86}$ (fig. 15b) e di Tomi-Costanza ${ }^{87}$ in Romania, ai quali si aggiungono anche i capitelli di Tessalonica, in particolare quelli già in opera nella Santa Sofia ${ }^{88}$.

Facevano quasi certamente parte dell'arredo liturgico del San Lorenzo altre sculture di marmo proconnesio riutilizzate nel San Ciriaco, come la colonnina in opera nella Cripta dei Protettori il cui esile fusto reca scolpita a bassorilievo una croce latina $^{89}$, e come il parapetto curvilineo di ambone

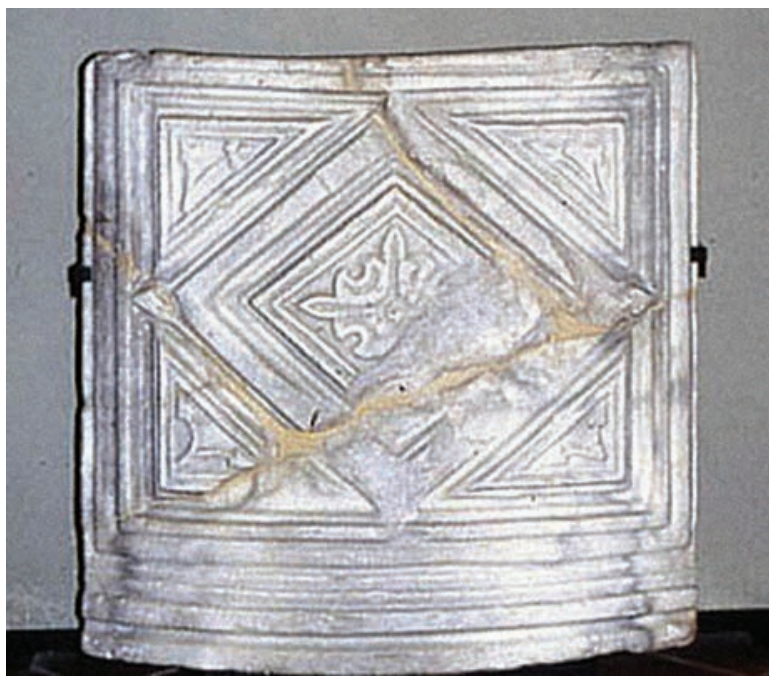

Fig. 16a. Ancona, Museo Diocesano: parapetto di ambone. oggi nel Museo Diocesano, il quale, in condizioni piuttosto malconce, esibisce le ferite inferte dai bombardamenti del 1943-4490 (fig. 16a). L'elegante decoro del parapetto, modulato sullo schema a modanature complesse con due quadrati concentrici posti diagonalmente, che trova un interessante raffronto in un parapetto nei depositi del Museo Archeologico d'Istanbul (fig. 16b), è stato più volte avvicinato al noto ambone di Sant'Apollinare Nuovo a Ravenna, considerato per lo più coevo alla costruzione della basilica, del quale condividerebbe pertanto anche la datazione ai primissimi anni del VI sec. ${ }^{91}$, dunque non troppo distante da quella dei capitelli.

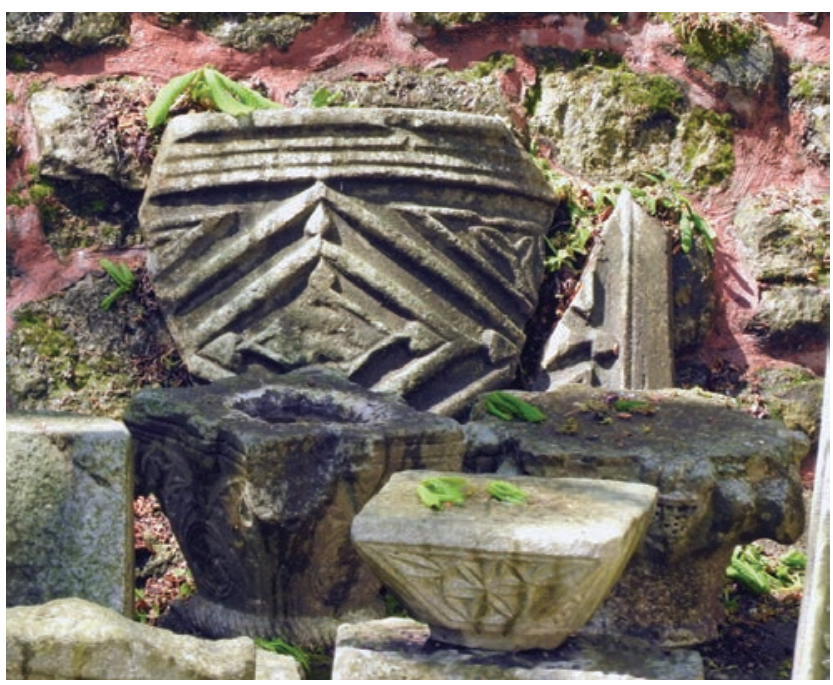

Fig. 16b. Istanbul, Museo Archeologico: parapetto di ambone.

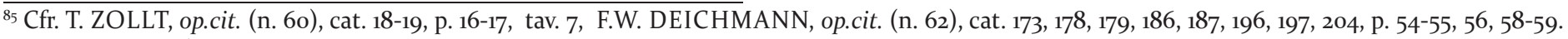
6o-61, 62, tav. 9-13).

${ }^{86}$ Il capitello, qui riprodotto, proveniente dalla basilica 23 (detta 'di Uvarov') di Cherson in Crimea, è conservato nel Museo Storico di Mosca: A. BORTOLI KAZANSKI, Répartition du marbre de Proconnèse en Crimée, in H. Ahrweiler (dir.), Geographica byzantina, Paris, 1981, p. 55-65, in part. p. 57-58, fig. 2; C. BARSANTI, L'esportazione op.cit. (n. 23), p. 156, fig. 83 (con bibliografia).

${ }^{87}$ Cfr. C. BARSANTI, L'esportazione op.cit. (n. 23), p. 156, fig. 84; assai vicina appare anche un'imposta del Museo di Silifke: A. AYDIN, op.cit. (n. 73), cat. 349-350, p. 283; Si veda anche V. VEMI, op.cit.(n. 72), p. 53.

${ }^{88} \mathrm{Si}$ vedano anche le imposte in opera e non nel San Demetrio: V. VEMI, op.cit.(n. 82), p. 78 e n. 128. 129, 133, p. 133-134, 133, tav. 42-43.

89 L. PANI ERMINI, op.cit. (n. 49), p. 108, fig. 27. Il fusto reca evidenti segni di scalpellature, riconducibili all'estesa ristrutturazione della cripta realizzata nel 1755 dall'architetto Varlé, quando tutti i supporti delle volte della cripta vennero rivestiti di marmi policromi.

${ }^{90}$ C. BARSANTI, Testimonianze...op.cit. (n. 22), p. 402; M. POLVERARI, op.cit. (n. 57), p. 14, fig. 11,6; C. BARSANTI, Mosaici... op.cit. (n. 25), p. 186 e nota 31; EAD, I plutei...op.cit. (n. 28), p. 454; Y.A. MARANO, op.cit. (n. 25), p. 166, fig. 4. Dallo storico Antonio Leoni (Istoria di Ancona Capitale della Marca Anconitana, II, Ancona, 1810, p. 239) sono in realtà ricordati due parapetti convessi che fino a poco tempo addietro si trovavano murati nella facciata del San Ciriaco e da dove, spiegava, erano stati rimossi per essere sistemati nel cortile del vescovado. Di essi fornisce anche una breve descrizione, riconoscendo nel loro decoro un «lavoro fine di greco scalpello, con motivi bizantini, rosa al centro, cornice romboide, fiori e gambi ai quattro angoli». In seguito, uno di quei parapetti venne trasferito nel lapidario allestito nella Cripta delle Lacrime, mentre l'altro, come riferisce, non senza amarezza, C. POSTI (op.cit. (n. 49), p. 12), era andato malauguratamente «sperduto per antiche e inveterate noncuranze».

${ }^{91}$ Cfr. C. BARSANTI, I plutei...op.cit. (n. 28), p. 454, fig. 231; B. VERNIA, L'arredo liturgico della basilica di Sant'Apollinare Nuovo a Ravenna, in C. Rizzardi (ed.), Venezia e Bisanzio. Aspetti della cultura artistica bizantina da Ravenna a Venezia (V-XIV secolo), Venezia, 2005, p. 363-389, in part. p. 363-367, fig. 3 e tav. XIV. 
Nel 1908, a completamento della vasta opera di restauro del San Ciriaco, si decise di rimuovere dalla facciata una serie di lastre figurate, già in opera nel parapetto della Cappella della Madonna, le quali, smontate in epoca barocca, erano state appunto murate in facciata, ai lati del maestoso protiro d'ingresso al tempio ${ }^{92}$. Si ebbe così modo di accertare che per quella con le figure dei Santi Liberio e Palatia, era stata riutilizzata una lastra di marmo proconnesio che recava sul retro una sobria decorazione modulata su esempi di tradizione paleocristiana. Sul liscio piano di fondo della lastra si staglia infatti una croce latina a estremità accentuatamente patenti incorniciata da un elegante motivo a treccia. Per la lastra, di fattura quasi certamente locale, identificata sia come paliotto d'altare, sia piuttosto come elemento di recinzione ${ }^{93}$, potrebbe essere suggerita una datazione tra la fine del VI secolo e i primi anni del secolo seguente.

Sconosciuta è invece la provenienza dell'isolato capitello corinzio del tipo 'a medaglione', di manifattura costantinopolitana, databile tra la seconda

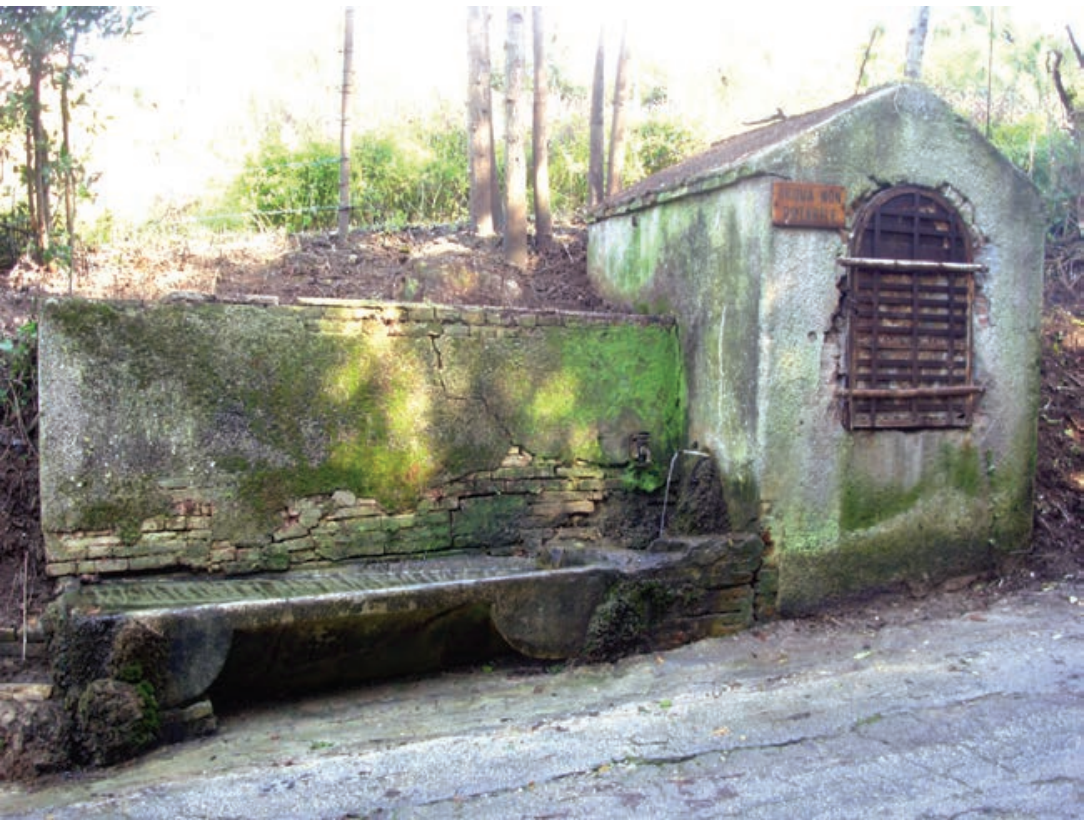

Fig. 17. Ancona: la Fonte delle monache in via delle Palombare.

celato dai decori barocchi realizzati dal Varlé nel 175695 . Essi, assieme ad un coperchio appartenente ad un sarcofago del medesimo tipo, utilizzato come abbeveratoio, la cosiddetta "Fonte delle monache" (fig. 17), lungo via delle Palombare ad Ancona ${ }^{96}$, attestano evidentemente la diffusione di questa categoria di manufatti prodotta ed esportata appunto dagli opifici del Proconneso tra II e V secolo ${ }^{97}$, testimoniata in area marchigiana anche da diverse repliche più economiche, realizzate, come quelle del Museo di Fano, in materiale lapideo ${ }^{9}$.

\footnotetext{
$9^{22}$ In tutto erano otto lastre con figure di santi e martiri venerati dalla chiesa anconetana: U. SCHNEIDER, Due recinzioni liturgiche medievali nel duomo di San Ciriaco di Ancona. Contributo alla storia dell'arte dell'incrostazione a Bisanzio e in Italia, in San Ciriaco...op.cit. (n. 21), p. 253-283; ed inoltre C. BARSANTI, Una nota sulla diffusione della scultura ad incrostazione nelle Marche medievali, ibidem, p. 284-288., 293; F. CODEN, Corpus della scultura ad incrostazione di mastice nella penisola italiana (XI - XIII sec.), Padova, 2006, p. 95-97, 381-396, tav. VI.3.1a, 2,7, 11-12,14-16.

${ }^{93}$ C. POSTI, op.cit. (49), p. 20; L. PANI ERMINI, op.cit. (n. 49), p. 107-108, fig. 24.

94 F. GUIDOBALDI, C. BARSANTI, A. GUIGLIA GUIDOBALDI, San Clemente...op.cit. (n. 26), p. 211, pp. 244-245, fig. 395; J. KRAMER, Spätantike korinthische Säulenkapitelle op.cit. (n. 78), p. 64 e 157-158; C. BARSANTI, Capitelli...op.cit. (n. 78), p. 1471.

${ }_{95}$ V. PIRANI, Il sarcofago di S. Ciriaco: ipotesi e certezze, in Presenza. Settimanale di informazione di Ancona e Osimo, 30.XI.1979; ID., op.cit. (n. 34) , p. 62-66, fig. 51-52 e 53. Rivolgo un grande ringraziamento a Mons. Ermanno Carnevali che ha condiviso con entusiasmo e agevolato con amichevole disponibilità le mie ricerche nel S. Ciriaco e non solo.

${ }^{96}$ La collocazione del fontanile viene per lo più erroneamente indicata in via Monte d'Ago: G. ANNIBALDI, Scavi e scoperte nel campo dell'archeologia cristiana in Ancona, in Atti II CNAC (Matera 1969), Roma, 1971, p. 98; S. SEBASTIANI, Ancona. Forma e urbanistica (Città antiche d'Italia, 4), Roma, 1996.p. 95, fig. 87. ${ }_{97}$ Cfr. B. RUSSELL, The Roman sarcophagus... op. cit. (n. 16).

${ }_{98}^{8}$ M.C. PROFUMO, Fano cristiana.... op.cit. (n. 26), p. 516.

* Referenze fotografiche: ove non altrimenti indicato, le fotografie sono di Claudia Barsanti.
} 


\section{NARBONNE ET LE NARBONNAIS DANS L'ANTIQUITÉ TARDIVE, CONTRIBUTION DE L'ÉTUDE DES ROCHES MARBRIÈRES À LA CONNAISSANCE DES OEUVRES SCULPTÉES.}

\section{ANNE-BÉNÉDICTE MÉREL-BRANDENBURG}

UDC: 711.453.4(448.7)"03/o7" 903.27:552.46

Original scientific paper

Manuscript received: 03. 04. 2016.

Revised manuscript accepted: 15. 04. 2016.

DOI: 10.1484/J.HAM.5.111344
A.-B. Mérel-Brandenburg Ecole du Louvre Paris, France benedicte.brandenburg@wanadoo.fr

The recurring questions about the identification of materials, especially the white marble, have led to the development of a program to identify materials by various analyses (size of the grains, accessory minerals, cathodoluminescence and isotopic analysis) with specific input from them. A systematic investigation concerning white marble artifacts of the Late Antiquity (IVth-VIII th centuries) in Narbonnais, (South of France) gathered 100 samples, give a better knowledge of the quarries centers, the probable location of execution of works, their dissemination area and the role of the port of Narbonne, in a region in contact with the Mediterranean and Atlantic worlds.

Keywords: Late Antiquity, Narbonne, port, trade, archeometry, marble quarries, workshops sculptors, capitals, sarcophagi.

Les recherches menées depuis une vingtaine d'années sur la sculpture de l'Antiquité tardive en Gaule méridionale ont mis l'accent sur l'apport de l'étude de l'origine des matériaux pour la détermination des ateliers, plus rarement sur l'appréciation des échanges commerciaux et des modes de transport. L'archéométrie conjuguée aux analyses typologiques, stylistiques, participe à la connaissance des modes de création et de circulation des œuvres en pierres marbrières destinées à la construction et au décor des monuments publics, privés et religieux, ou à caractère funéraire en narbonnais, dans une région en contact avec la Provence, l'Aquitaine, la Péninsule ibérique - et plus lointaine - l'Italie.

\section{MÉTHODES POUR UNE ÉTUDE}

Les examens pétrographiques pratiqués sur le terrain et en laboratoire, précisent objectivement la nature et la provenance des pierres à 95\% marbrières (fig. 2). Un programme

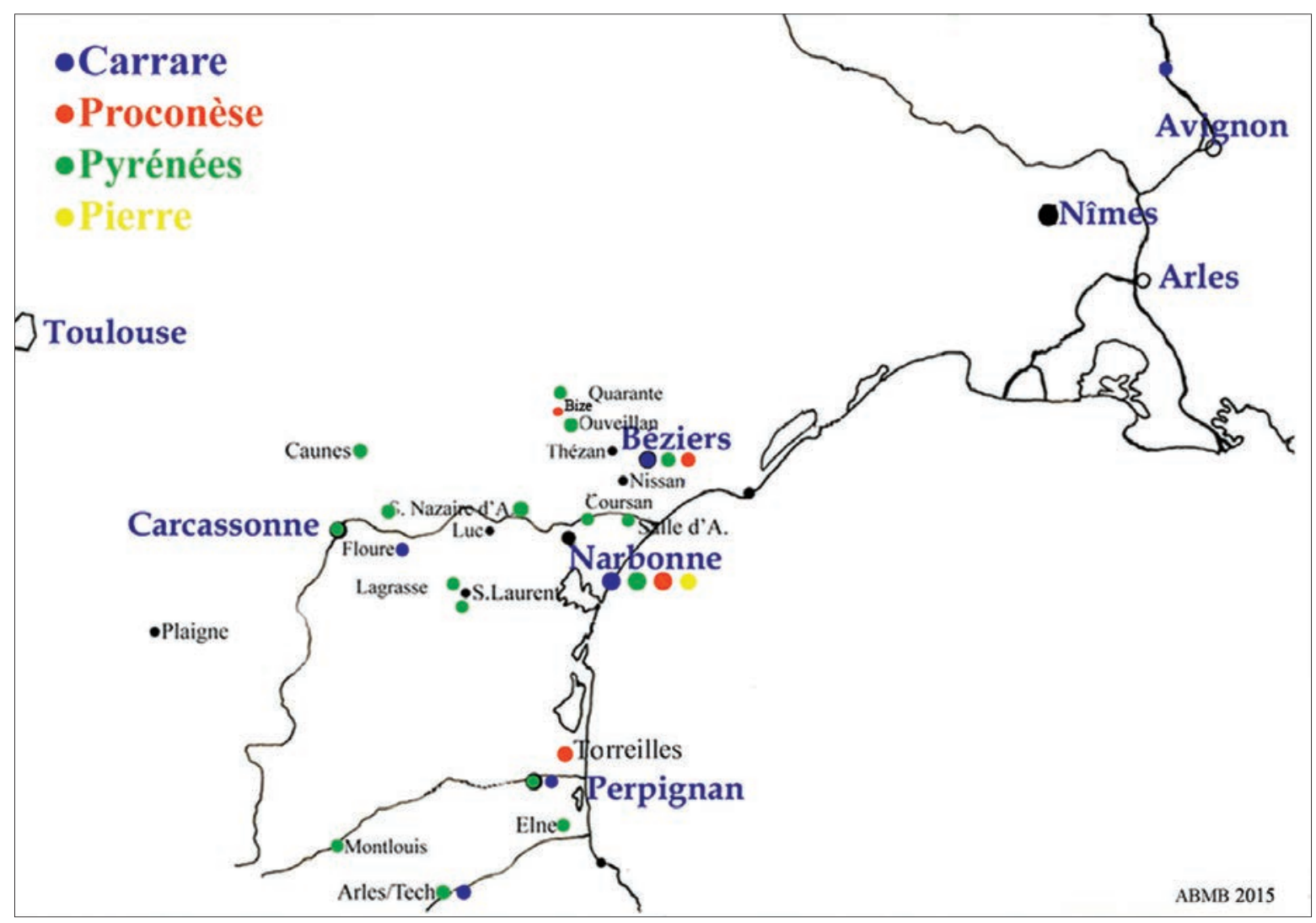

Fig. 1. Carte du Languedoc et lieu de conservation des ouvres (C) Mérel-Brandenburg 


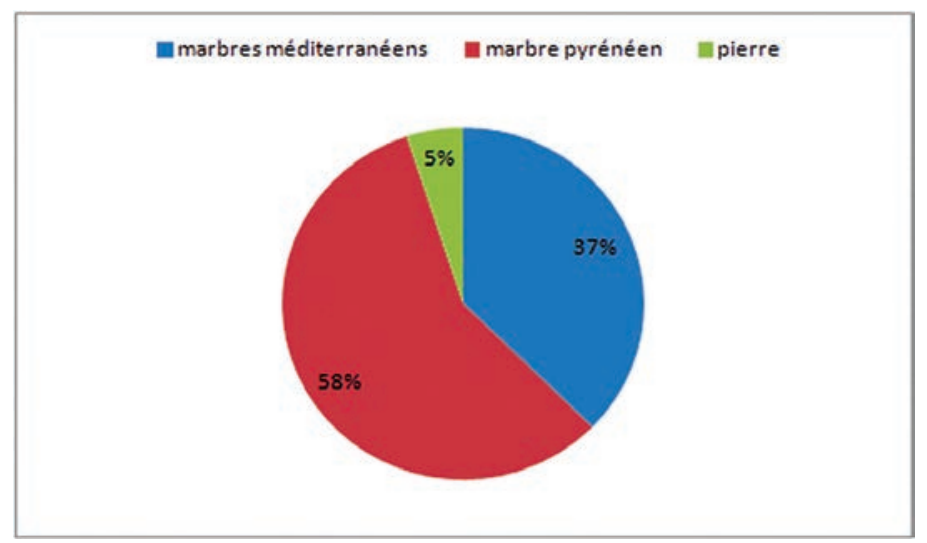

Fig. 2. Graphique : proportion des matériaux. @Mérel-Brandenburg Rapport proportionnel analytique des marbres. CMérel-Brandenburg

de recherche a été réalisé en Languedoc ${ }^{1}$ dans la lignée des études conduites par C. Costedoat et J. Cabanot sur les chapiteaux et les sarcophages du Sud-Ouest de la Gaule ${ }^{2}$, par M. Immerzeel sur les sarcophages à frise de la région de Saint-Béat et d'une première enquête menée en Languedoc par J.- C. Richard avec Ph. et A. Blanc ${ }^{3}$.

Ce travail a pris une dimension originale par le nombre d'œuvres analysées, plus de 300, et leur diversité fonctionnelle : éléments architectoniques, mobilier liturgique, et monuments funéraires. Une centaine d'œuvres localisée à Narbonne et sur son territoire a été retenue (fig. 1). Elles sont datées du $1^{\mathrm{er}}$ tiers du IV à la fin du Ve $\mathrm{s}$. Cette sélection tient compte du contexte archéologique et de leur histoire, certaines ayant été déplacées soit à l'époque médiévale ${ }^{4}$ ou moderne ${ }^{5}$, soit par le biais des collectionneurs ${ }^{6}$. Les matériaux non identifiés ${ }^{7}$, non analysés ${ }^{8}$ ou recyclés ont été exclus $^{9}$ (fig. 3).

\section{ANALYSE DES MARBRES}

Les blocs ont été analysés suivant un protocole, établi par Ph. Blanc (marbre) et A. Blanc (pierre), géologues, identique à celui mis en œuvre pour caractériser les échantillons provenant des carrières.

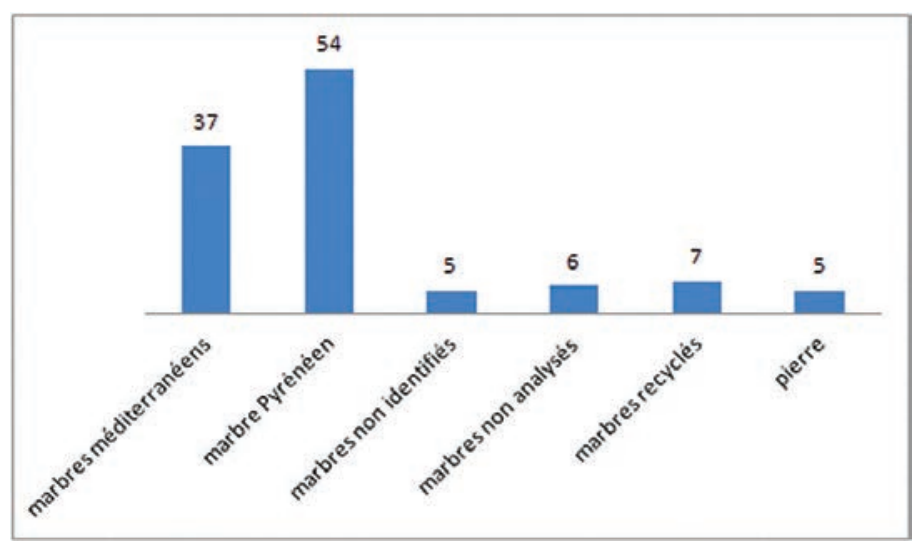

Fig. 3. Graphique : auvres retenues.

Le principe de base permettant de distinguer les marbres est à priori simple. Le marbre résulte de l'action de fortes pressions et/ou de températures élevées affectant les roches carbonatées (calcaire-dolomites) présentes dans l'écorce terrestre. Il en découle une réorganisation des cristaux de carbonates qui transforme la roche en marbre (processus de métamorphisme) en lui conférant une texture, une structure cristalline, une signature isotopique en carbone et en oxygène propres au gisement. L'absence de fiabilité de l'identification des marbres blancs par examen macroscopique (couleur et grain) et par analyse pétrographique a conduit, à partir des années 1960-1970 ${ }^{10}$, les équipes de chercheurs à appliquer de nouvelles techniques d'analyses, dont le croisement des résultats participeà une détermination probable de l'origine des marbres.

\section{Évaluation de la taille maximum des grains}

La première étape est un examen visuel du bloc à la loupe et à la lumière pour en mesurer la diffusion. Elle détermine la taille et la forme des grains de calcite et précise les faciès (fig. 4). Elle comprend un descriptif et une couverture photographique, et le prélèvement d'une esquille ${ }^{11}$ destinée aux études en laboratoire ${ }^{12}$.

${ }^{1}$ Programme de recherche, UMR 8167 sur L'origine des matériaux utilisés pour les monuments sculptés de l'Antiquité tardive en Languedoc méditerranéen. A.-B. MÉREL-BRANDENBURG, A. BLANC, Ph. BLANC, De l'origine des matériaux utilisés dans les monuments sculptés de l'Antiquité tardive en Languedoc méditerranéen, in Marbres et autres roches de la Méditerranée antique : études interdisciplinaires : VIIIe international conference of the Association for the study of marble and other stones used in antiquity (ASMOSIA), Aix-en-Provence, 12-18 juin 2006, Aix-en-Provence, 2009, p. 421-438.

${ }^{2} \mathrm{~J}$. CABANOT et C. COSTEDOAT, Recherche sur l'origine du marbre blanc utilisé pour les chapiteaux et les sarcophages de l'Antiquité et du haut-moyenâge, in Aquitania, XI, 1993, pp. 189-232.

${ }^{3}$ J.-C. RICHARD, Les sarcophages en marbre blanc du Languedoc-Roussillon : une enquête en cours, in Revue Archéologique de la Narbonnaise, 33, Paris, CNRS, 2000, p. 286-289 ; J.-C. RICHARD, Les sarcophages en marbre blanc, "sarcophage de lécole d'Aquitaine" en Languedoc-Roussillon, in Actes du colloque, Pierre et Archéologie, Tautavel, 14-16 mai 1998, J.-C. Miskovski, J. Lorenz (dir.), Perpignan, Presses Universitaires de Perpignan, p. 69-80 ; A. BLANC, Ph. BLANC, J.-C. RICHARD, Comparison between white marble sarcophagi and other carved objects from Languedoc (France), in Asmosia 5 : Interdisciplinary Studies of Ancient Stone, Archetype Publications Ltd, London, 2002, p. 201-205

${ }^{4}$ Abbaye de Lagrasse (Aude), Arles-sur-Tech (Pyrénées-Orientales), Caune-Minervois (Aude)

${ }^{5}$ Plaigne (Aude), Elne (Pyrénées-Orientales) ?, Toreilles (Pyrénées-Orientales) ?

${ }^{6}$ Montlouis (Pyrénées-Orientales), Perpignan ( Pyrénées-Orientales), Quarante (Hérault)

${ }_{7}$ Plaque de chancel dite du triomphe de la Croix, plaque dite de la chasse mystique, dalle au paon (Narbonne, musée archéologique)

${ }^{8}$ Les chapiteaux remployés dans le clocher de l'église abbatiale de Caunes-Minervois inaccessibles ou appartenant à des collections particulières.

9 Blocs architectoniques réutilisés en support reliquaire : Support de Céleyran (Narbonne, musée archéologique), d'Oupia (Hérault), d'Ouveillan (Aude) église ; Dalle funéraire de Siniofreda (Narbonne, musée archéologique), en élément de chancel ou devant d'autel : Pézilla-la-Rivière (Pyrénées-Orientales) ; fûts de colonne creusés en sarcophage provenant de l'Abbaye de Lagrasse (Aude), conservéà Perpignan, rue de la Réal, ou transformés en support-reliquaires à Saint-Nazaire-d'Aude (Aude)

${ }^{10}$ Voir CABANOT 1993 : p. 191, note 8 ; IMMERZEEL, JONGSTE 1994 : p. 248, note 83.

"1 10 milligrammes de marbre à l'aide d'un petit burin, dans un emplacement non visible. Les esquilles sont broyées pour fabriquer des lames transparentes ou des pastilles.

${ }^{12}$ Les analyses en cathodoluminescence et des isotopes stables ont été réalisées à l'Université Pierre et Marie Curie (Paris 6), par Ph. Blanc et N. Labourdette (analyste). 


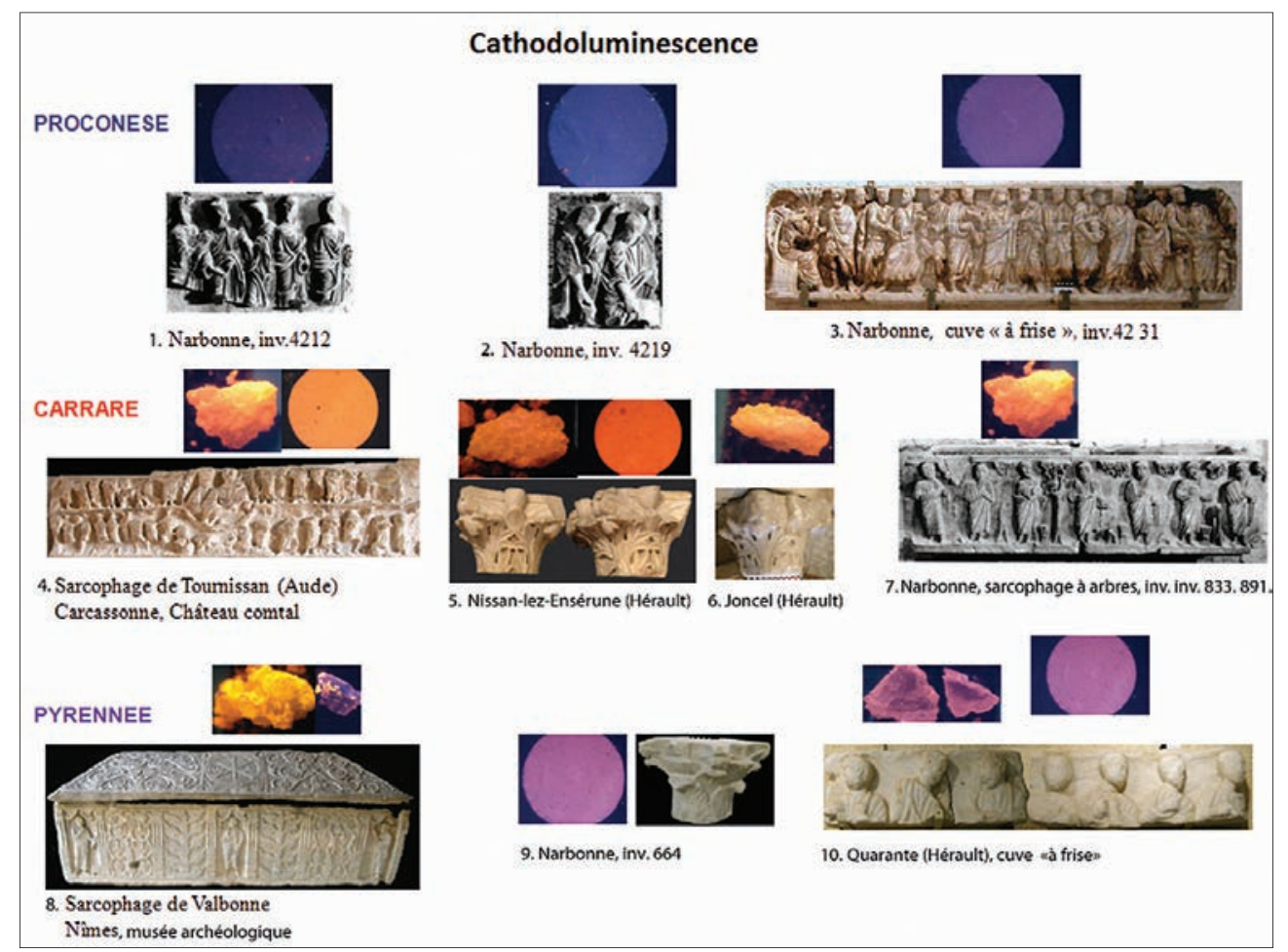

Fig. 6. Résultat de l'étude par cathodoluminescence d'euvres narbonnaises (C) Ph. Blanc

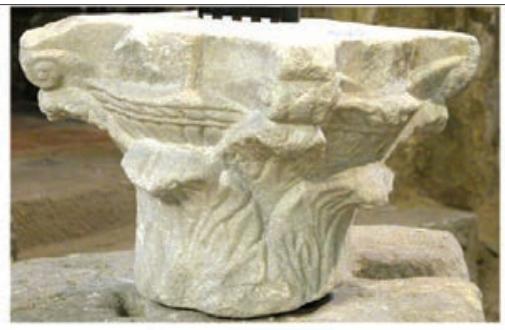

1
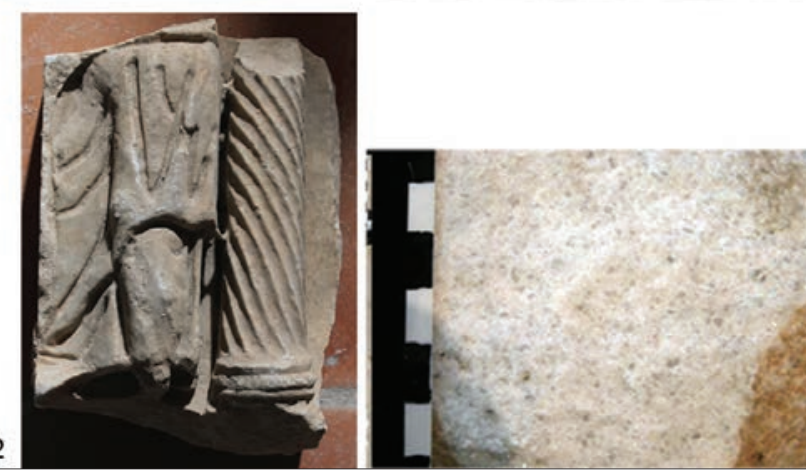

Fig. 4. Taille des grains: 1. Chapiteau, marbre blanc gris à gros grains Narbonneinv.664 ; 2. Fragment de cuve provenant des fouilles de l'église Saint-Félix, marbre à grains fins de Carrare. (C) Ph. Blanc

La dimension des grains de marbre qui résulte des conditions du métamorphisme, caractéristique de chaque gisement (MGS) ${ }^{13}$, est précisée par un examen au microscope optique (fig. 5). La méthode offre un paramètre quantitatif rapide pour définir la texture qui est comparée avec les diverses bases de données recensant les variations de la taille maximum des grains de marbre des carrières de marbre

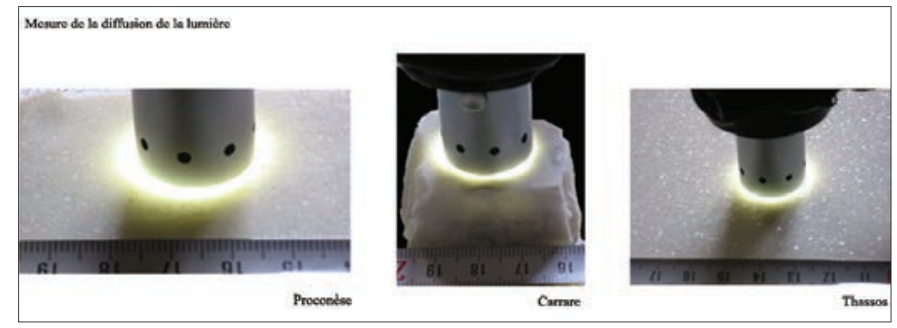

Fig. 5. Évaluation de la taille maximum des grains : mesure de la diffusion de la lumière optique. (CPh. Blanc

blanc du monde classique. Les résultats conduisent à une première identification des faciès et sont complétés par des indications obtenues par cathodoluminescence

\section{Cathodoluminescence}

Cette technique consiste à observer la lumière émise après excitation de la surface du matériau par bombardement d'un faisceau d'électrons sous haute tension dont l'intensité d'émission résulte de la concentration en $\mathrm{Mn}^{2+}$ dans le carbonate ${ }^{14}$. Le spectre permet d'établir, en fonction des couleurs, un catalogue d'échantillons qui distingue les marbres dolomitiques des calcitiques, chaque minéral se caractérisant par une couleur propre ${ }^{15}$ : les fortes concentrations apparaissent du jaune à l'orangée, les faibles, brunes et les très faibles, bleues (Proconnèse). Les différentes carrières de Proconnèse possèdent la même luminescence majeure bleue correspondant à des concentrations en manganèse très faibles (fig. 6). A contrario, les marbres des Pyrénées

\footnotetext{
${ }^{13}$ Maximum Grain Size.

${ }^{14}$ Pour les méthodes appliquées voir : Ph. BLANC, A. BLANC, Identification des matériaux des sarcophages païens d’Arles. La méthode de cathodoluminescence, in V. GAGGADIS-ROBIN, op. cit. Annexe II, p. 287-291 : l'intensité d'émission est due à la concentration en $\mathrm{Mn}^{2}+$ dans le carbonate, émet une lumière orangée, à $620 \mathrm{~nm}$, alors que la dolomite que l'on rencontre en particulier dans le marbre de Thassos ou en inclusion dans d'autre comme celui marbre de Sost, émet une lumière franchement rouge, à $650 \mathrm{~nm}$.

${ }^{15}$ Les images de CL des grains ont été contrôlées ou précisées par des analyses spectrales mesurant les longueurs d'onde spécifiques. Enfin, on a mis en évidence des différences significatives du temps de pose pour les prises de vues des rayonnements correspondant aux divers marbres.
} 


\section{Minéraux accessoires}

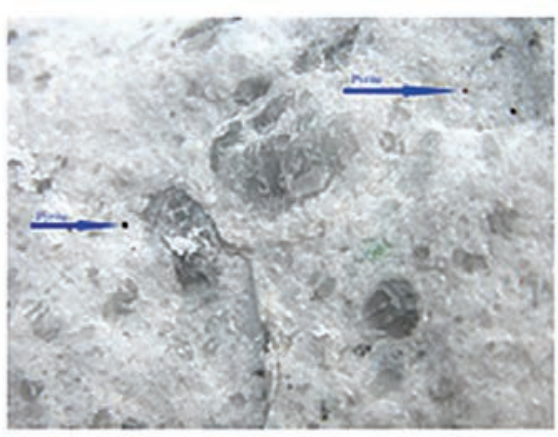

Association de grains fins et grossiers

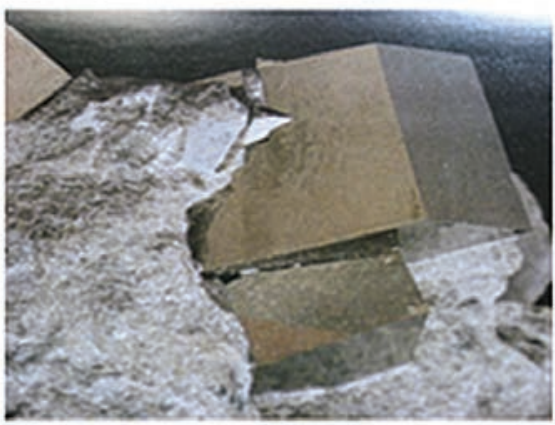

Cristaux de pyrites

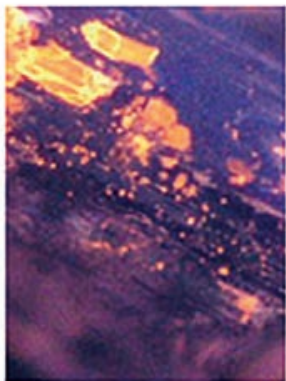

Scapolite

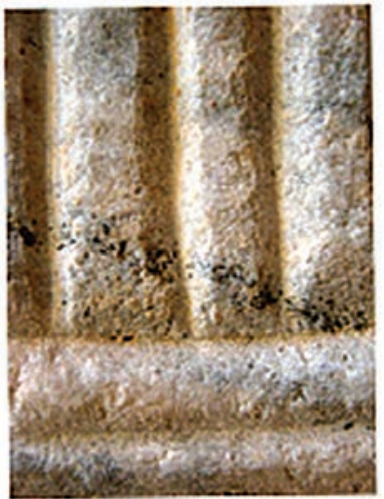

Graphite

Fig. 7. Mise en évidence des minéraux accessoires marqueurs des marbres des Pyrénées (C) Ph. Blanc.

peuvent contenir un minéral accessoire marqueur : la scapolite ${ }^{16}$ (fig. 7).

\section{Analyse des isotopes stables}

Le résultat des trois analyses précédentes définit les propriétés du marbre, sans différencier certains marbres des Pyrénées ${ }^{17}$ de ceux du Proconnèse qui offrent des particularités communes lors d'un premier examen : larges veines grises parallèles, dégagement gazeux odorant lors du prélèvement (Proconnèse). Le recours à la mesure des rapports des isotopes stables ${ }^{18} \mathrm{du}$ carbone $\left(\mathrm{C}_{13} / \mathrm{C}_{12}\right.$ en $\left.\delta^{13} \mathrm{C}\right)$ et de l'oxygène $\left(\mathrm{O}_{18} / \mathrm{O}_{16} \text { en } \delta^{18} \mathrm{O}\right)^{19}$ contenus dans les carbonates, méthode considérée au début comme très efficace, s'est révélée progressivement inappropriée en raison de la superposition des champs isotopiques les représentant ${ }^{20}$ (fig. 8).

L'examen pétrographique et l'analyse par cathodoluminescence ont été effectuées systématiquement et les résultats ponctuellement vérifiés par d'autres procédés : nettoyage au laser mettant en évidence des auréoles grises sur un marbre de Carrare (fig. 9), analyse des isotopes pour le Carrare.

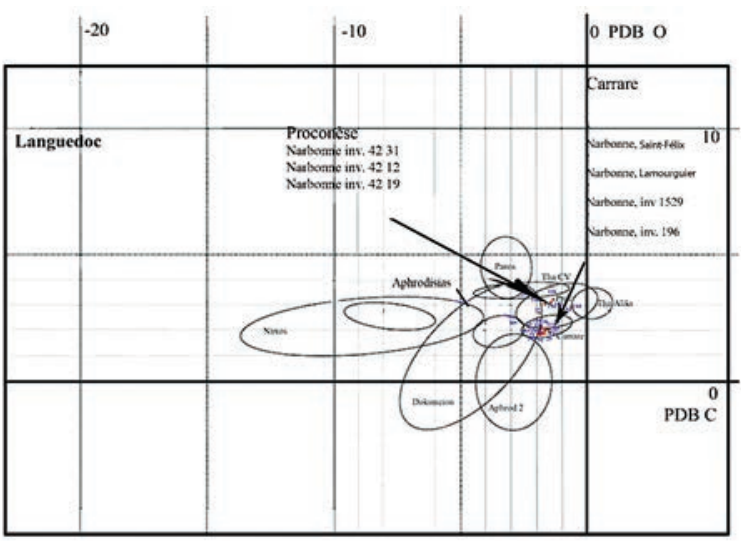

Fig. 8. Analyse des isotopes stables. (CPh. Blanc

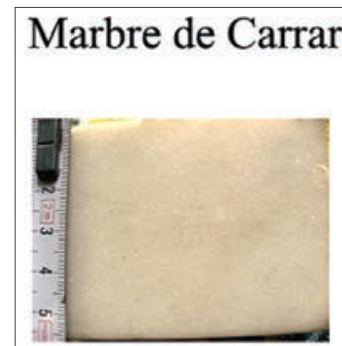

Grains fins

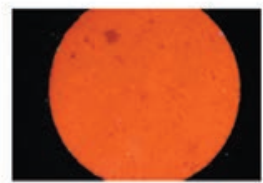

CL jaune orangé

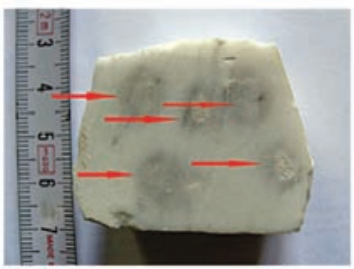

Impact au laser de nettoyage

Fig. 9. Nettoyage au laser d'un marbre de Carrare (CPh. Blanc

Sachant qu'aucune méthode n'est fiable à 100\%, seule la convergence ou la complémentarité des données apportent des assurances satisfaisantes.

Quatre types de pierres marbrières ont été identifiés comme provenant soit des carrières méditerranéennes de Carrare, de Proconnèse, de Thassos, soit des carrières de Saint-Béat dans les Pyrénées, exploitées de longue date. La production est caractérisée par un usage important des marbres des Pyrénées (59\%) et de Carrare (34\%), plus limitée de Proconnèse $(6 \%)$ et rare de Thassos $(1 \%)$. Les marbres de Thassos et de Proconnèse ont été utilisés uniquement pour des sarcophages, celui de Carrare pour des sarcophages, des éléments architectoniques, en moindre quantité pour du mobilier liturgique ${ }^{21}$ L'emploi du marbre des Pyrénées est remarquable par le nombre de sarcophages et quelques éléments architecturaux ${ }^{22}$ (fig. 10).

\footnotetext{
${ }_{16}$ [AISi3O8] $\mathrm{Na} 2-\mathrm{Ca}, \mathrm{Cl}$.

${ }^{17}$ Les marbres des Pyrénées sont souvent à grains moyens à gros avec des macles et des clivages courbes. Les minéraux accessoires symptomatiques, sont accompagnés de graphite, et/ou scapolite. Au broyage se produit un dégagement gazeux à odeur forte. La CL est variable de rose à brun orangé.

${ }^{18}$ Voir Y. MANIATIS et al., Techniques utilisées pour déterminer la provenance de quelques sarcophages en marbre du musée de l’Arles et de la Provence antique, in : V. GAGGADIS-ROBIN, op.cit., Annexe I, p. 281.

${ }^{19}$ Chaque marbre possède une signature isotopique en carbone et en oxygène propre au gisement.

${ }^{20}$ Lorsque le matériau provient de Proconèse, de Carrare et de Paros, le champ très restreint et la couleur de luminescence spécifiques lèvent le doute.

${ }^{21}$ Table d'autel dédicacée par l'évêque Rusticus, conservée dans l'église Minerve, table d'autel de Bize-Minervois (Aude).

${ }^{22}$ Chapiteaux, linteaux de la cathédrale et de l'église Saint-Félix de Narbonne, de l'église du prêtre Othia sur le piémont de l'oppidum d'Ensérune (Hérault), reliquaire du Saint Sépulcre.
} 


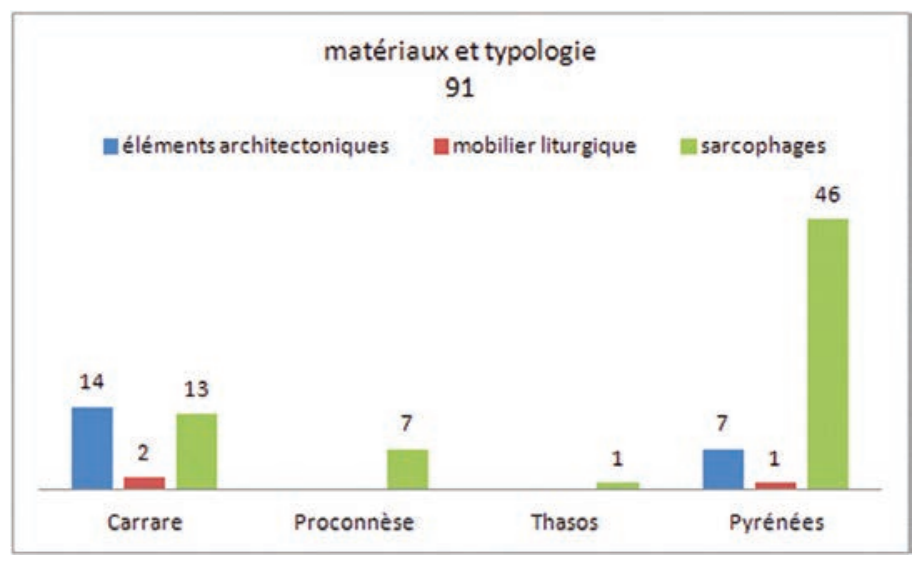

Fig. 10. Proportion des œuvres sculptées en marbres méditerranéens et Pyrénéen. (C)érel-Brandenburg

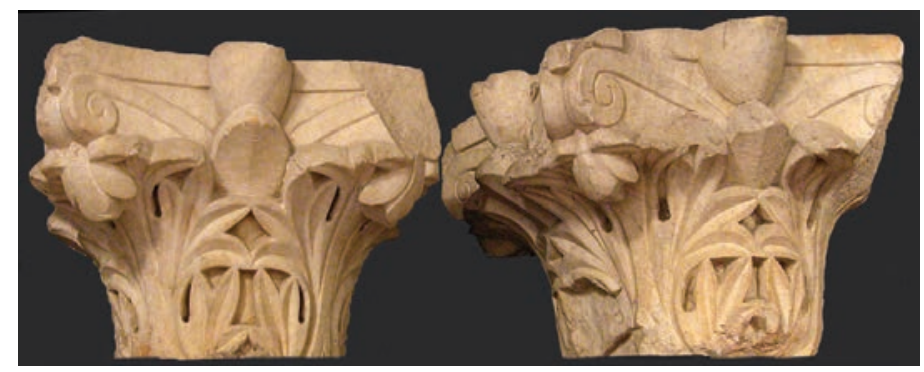

Fig. 12. Chapiteaux composites à feuilles d'acanthe épineuse, marbre de Carrare, Nissan-lez-Ensérune, église. (CPh. Blanc

son étude sur les chapiteaux de marbre du haut Moyen âge de Saint-Denis ${ }^{26}$, doit être nuancée. Les chapiteaux, tous inspirés des modèles antiques se rattachent au groupe dérivé du composite à feuilles d'angle aux variantes ornementales

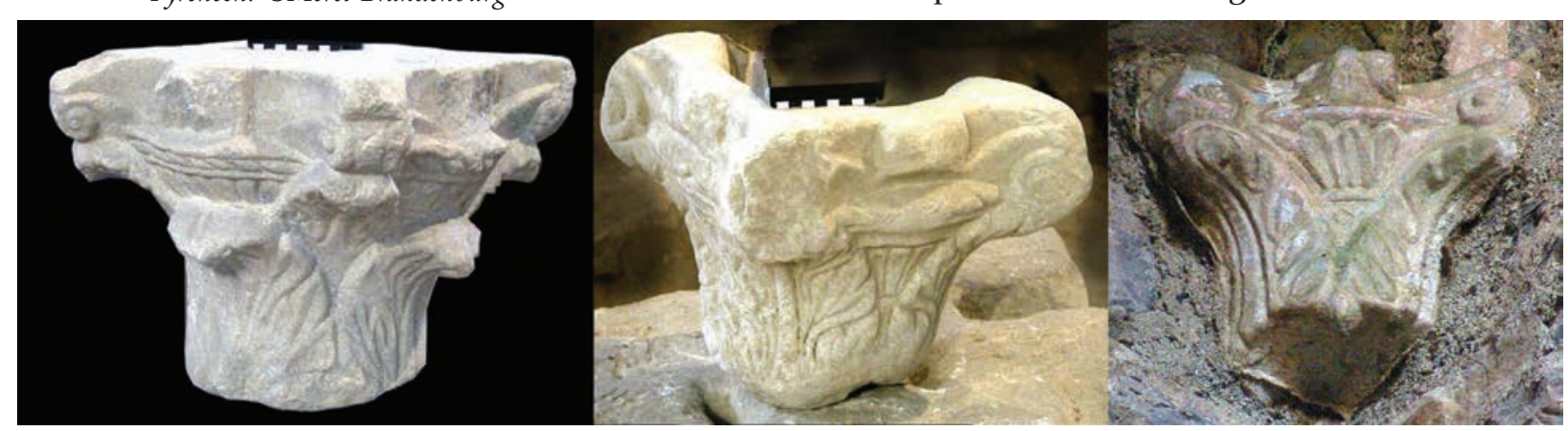

Fig. 11. Chapiteaux, marbre des Pyrénées: 1. chapiteau dérivé du composite type 1, Narbonne, Lapidaire, inv. $664 ; 2$. Chapiteaux dérivés du composite à feuilles d'angle, type 3, Narbonne, Lapidaire; 3. Chapiteaux dérivés du composite à feuilles d'angle, type 3, Lagrasse, église abbatiale (CMérel-Brandenburg

Aucun document n'assure l'exploitation des carrières de pierres marbrières du Languedoc au cours de l'Antiquité tardive. La thèse de leur utilisation développée par E. James était fondée jusqu'à une date récente sur des faits historiques, liés la conjoncture politique et économique du Sud-Ouest de la Gaule. Elle résulterait de la rupture des relations commerciales entre l'Aquitaine et la Septimanie provoquée par la conquête franque, au début du $\mathrm{VI}^{\mathrm{e}} \mathrm{s} \cdot{ }^{23}$ et par les menaces basques dans les Pyrénées auraient entrainé des difficultés d'accès aux carrières de la Vallée de la Haute-Garonne, vers la fin du VI ${ }^{\mathrm{e}} .^{24}$.

\section{ATELIERS}

La mise en perspective des résultats des analyses des matériaux, de la technique de taille, des schémas de composition, des motifs préférentiels et du traitement des œuvres, remet en question les attributions d'atelier, au regard de propositions anciennement formulées ou récentes : ateliers romains ou provinciaux - toulousains ou narbonnais-.

La thèse de l'exclusivité de la production des chapiteaux par les ateliers du Sud-Ouest retenue depuis les travaux de Jean-Hubert ${ }^{25}$, reprise par May Vieillard-Troïekouroff dans nombreuses. Un premier groupe se caractérise par des chapiteaux en marbre des Pyrénées exécutés dans les ateliers du Sud-Ouest : chapiteaux dérivés du corinthien type 2, chapiteaux composites à syntaxe canonique type 2 , chapiteaux dérivés du composite à feuilles d'angle, de type 1 et de type 3, par référence aux travaux de $\mathrm{D}$. Tardy et $\mathrm{C}$. Balmelle ${ }^{27}$ (fig. 11). Un second se compose de chapiteaux aux dimensions variables, définis par la composition ornementale, à double couronne imbriquée (type 3 ) ou à feuilles d'angle et double dé (fig. 12), taillés en biseau et en réserve de l'acanthe épineuse couvrant la totalité de la corbeille. La structure de la corbeille et de l'abaque est comparable aux chapiteaux composites tardifs d'Ostie publiés par P. Pensabene ${ }^{28}$, or le traitement, d'une qualité plastique supérieure, s'apparente aux chapiteaux « à masques », en Proconnèse localisés sur le pourtour du bassin méditerranéen ${ }^{29}$. Leur originalité résulte de l'emploi du marbre de Carrare et de la présence, sur la partie supérieure de la corbeille, d'un élément médian saillant correspondant à la simplification géométrique de l'extrémité d'une feuille d'où prennent naissance les canaux des volutes. Leur distribution géographique, avec une densité maximale dans la région de Narbonne, atteste une production micro-provinciale en narbonnais. Ces analyses

${ }_{23}$ La bataille de Vouillé en 507, victoire de Clovis sur Alaric II, entérina la défaite des Visigots et leur repli vers la Septimanie puis l'Hispanie.

${ }^{24}$ E. JAMES, The Merovingian Archaeology of South-West Gaul, Oxford, 1977, (BAR, 25), p. 51-61.

${ }^{25}$ J. HUBERT, L'art pré-roman, Paris, 1938.

${ }^{26}$ M. VIEILLARD-TROÏEKOUROFF, Les chapiteaux de marbre du Haut Moyen-âge à Saint-Denis, in Gesta, XV-1 et 2, pp. $105-112$.

${ }^{27}$ C. BALMELLE, Les demeures aristocratiques d'Aquitaine : société et culture de l'Antiquité tardive dans le Sud-Ouest de la Gaule, Pessac, 2001 (Aquitania, suppl. 10).

D. TARDY, Les transformations des ordres d'architecture : l'évolution du chapiteau composite en Aquitaine au Bas-Empire, in Aquitania, 14, 1996, 183-192. ${ }^{28}$ P. PENSABENE, Scavi di Ostia, VII, 1. Capitelli, Rome, 1973. p. 105, n³ 383, p. 160, nº57

${ }^{29}$ A. PRALONG, Les chapiteaux corinthiens tardifs en marbre de Proconèse : une nouvelle typologie, in Bulletin de l'Association pour l'Antiquité tardive, 13, 46-53. Le modèle se structure dès le $\mathrm{V}^{\mathrm{e}} \mathrm{s}$., avant d'être fabriqué en quantité et diffusé. 


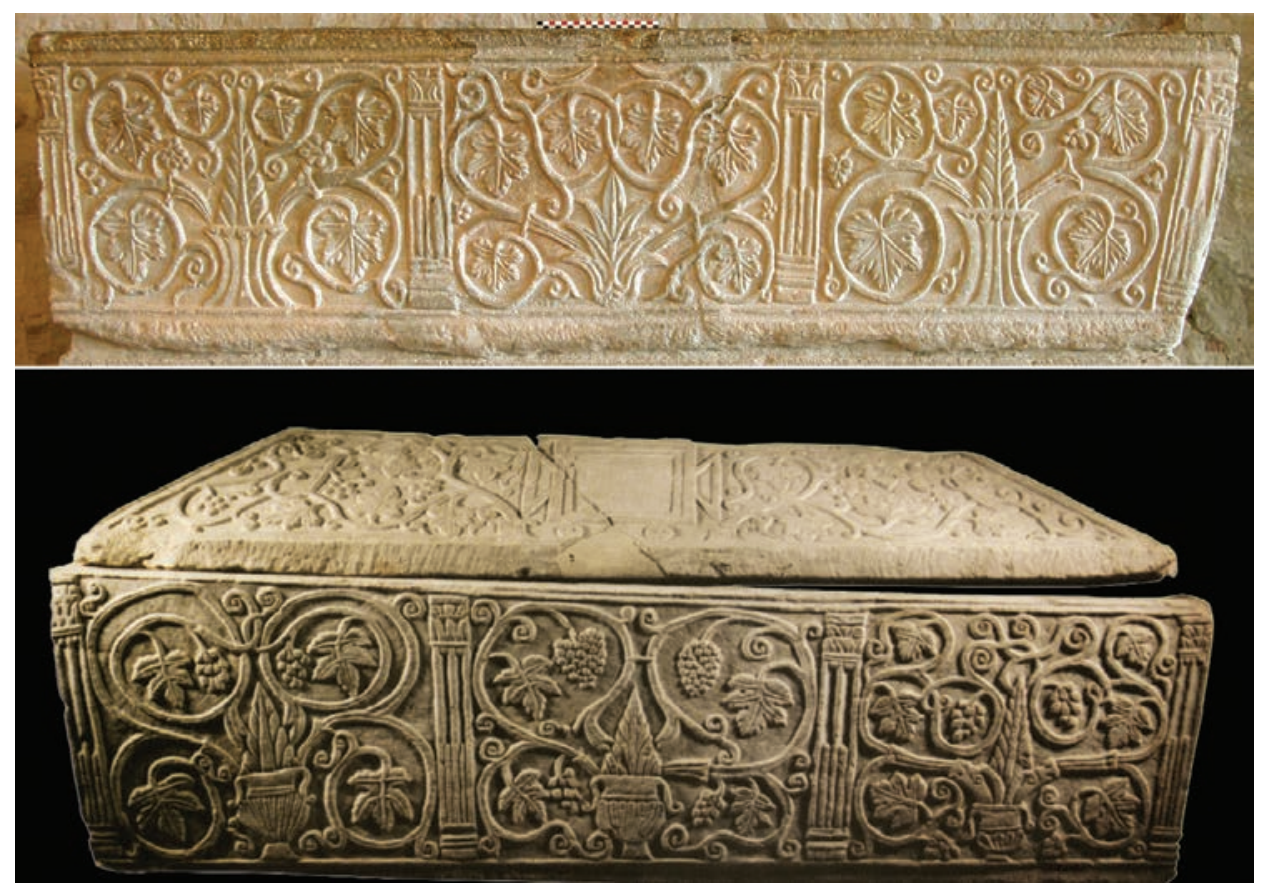

Fig. 13. Cuves panneautées à décor végétal, $2^{e}$ moitié $I V^{E}$-déb. V s., marbre des Pyrénées : 1. provenant de Floure (Aude), Carcassonne, Château comtal. CMérel-Brandenburg; Toulouse, musée Saint-Raymond, inv.RA14. Cmusée Saint-Raymond.
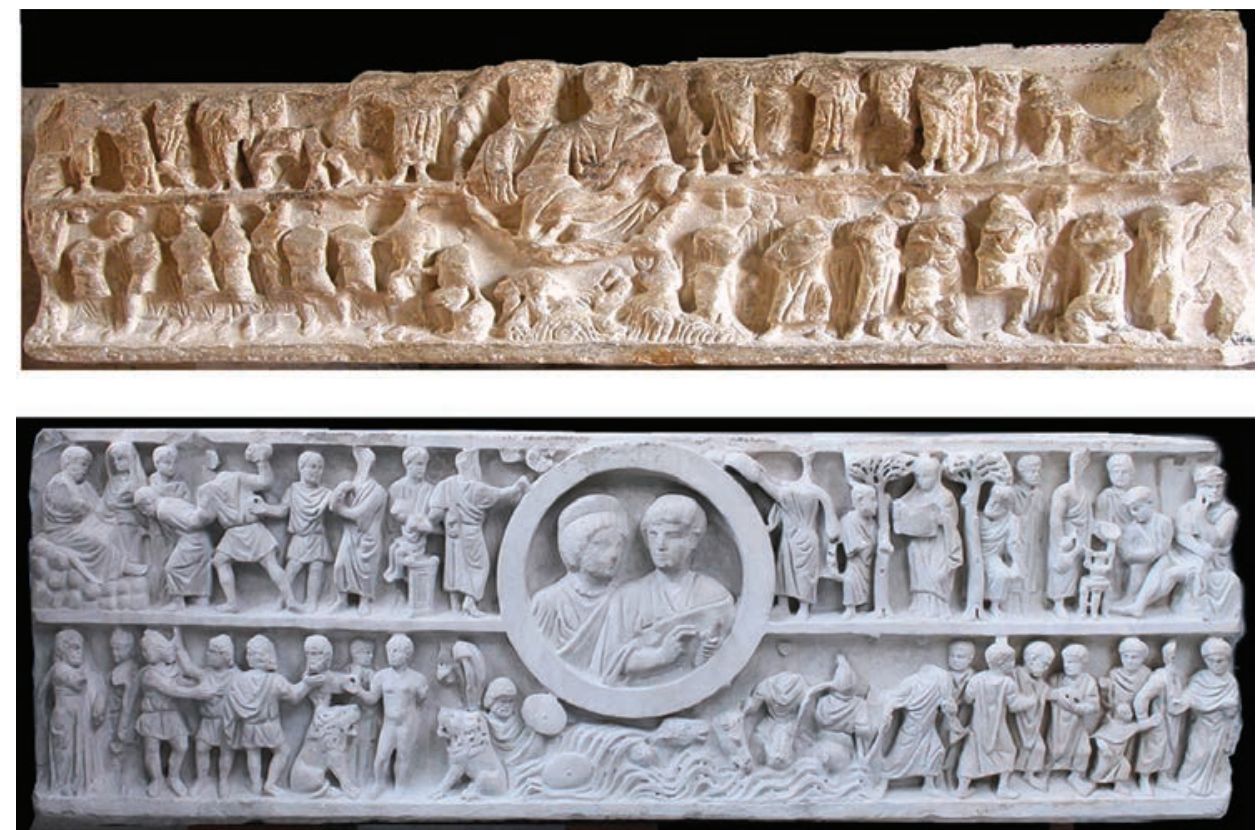

Fig. 14. Sarcophages à double registre, milieu IVe s., marbre de Carrare : 1. Tournissan, Carcassonne, Château comtal, inv.6. CMérel-Brandenburg; 2. Arles, Sarcophage dit de la Chaste Suzanne, Arles, musée de l'Arles antique. Ctous droits réservés.

confortent la diversité des matériaux utilisés pour les chapiteaux tardo-antiques ${ }^{30}$.

Les sarcophages représentent la majorité du matériel et sont définis par une production en marbres méditerranéens de types variés avec des strigiles, à frise continue, à registres superposés, à scènes séparées par des arbres ou des colonnettes (fig. 6. 1-2-3-4-7-10), et une autre dominante en marbre de Saint-Béat, innovant par l'alliance de personnages et d'un décor végétal luxuriant (fig. 6.8, fig.13).

Les sarcophages à décor figuratif en marbres méditerranéens ont été exécutés dans les officines romaines. Certains généralement attribués à des ateliers provinciaux ont été importés de Rome. Citons la cuve à registres superposés et médaillon médian découverteà Tournissan (Aude) (fig. 14.1), reconnue comme une « œuvre gauloise » par F. Benoit ${ }^{31}$, à partir de l'identification erronée du marbre provenant soit de Saint-Pons-de-Thomières (Hérault) ou des Pyrénées. Le recours au marbre de Carrare, la composition en registre et les thèmes illustrés - Passage de la mer Rouge, imago des époux- très proches de la cuve dite de la Chaste Suzanne en Arles, la rattachent à la production romaine (fig. 14.2). Il en est de même des cuves fragmentaires ornées des apôtres et

${ }^{30}$ A.-B. MÉREL-BRANDENBURG, P. BLANC, A. BLANC, Espace architectural, décor sculpté et matériaux en Languedoc méditerranéen au cours de l'Antiquité tardive, in Décors et architecture en Gaule entre l'Antiquité et le haut Moyen Âge : mosaïque, peinture, stuc, C. Balmelle, H. Eristov, F. Monier (dir.), Pessac, 2010 (Aquitania, suppl. 20), p. 675-688.

${ }^{31}$ F. BENOIT, Les sarcophages paléochrétiens d'Arles et de Marseille, Paris, 1954 (Gallia, suppl. 5), p. 20. 


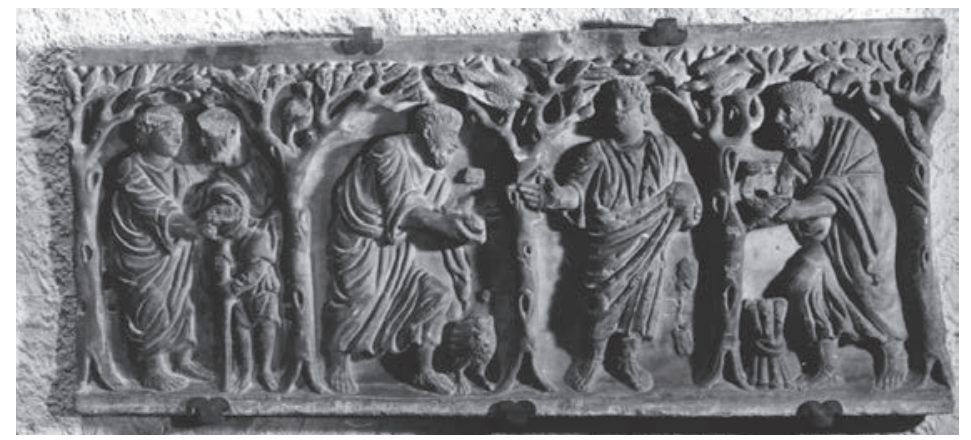

Fig. 15. Cuve à arbres, deuxième moitié IVe s., marbre de Proconèse, Narbonne, musée archéologique inv. 855.3.3. (C) D. Fourmont).

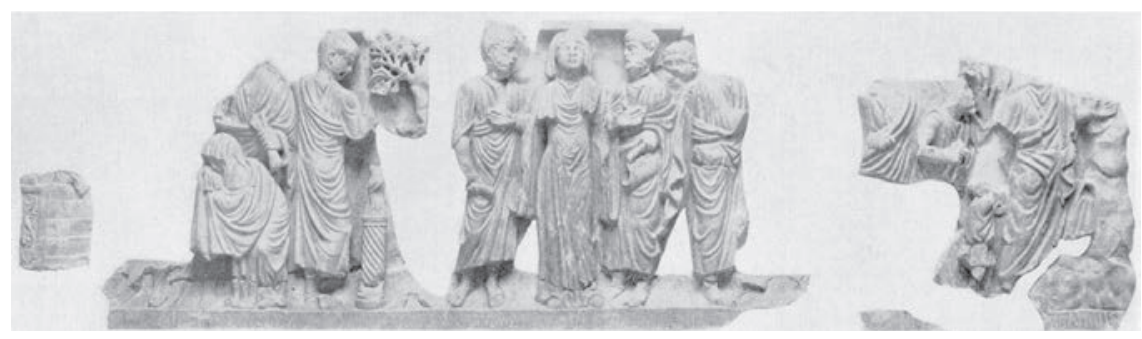

Fig. 16. 2. Rome, cimetière de Prétextat, sarcophage de Flavia Sabina (Repertorium I)

de scène des deux Testaments ${ }^{32}$ (fig. 6. 1 et 2), et «à arbres » (fig. 15), en marbre de Proconnèse. L'exécution des personnages (visages, drapés) relèvent de modèles romains. À ces exemples significatifs s'ajoute la cuve de Narbonne (inv. 4218), sur laquelle figurent la Guérison de l'Hémorroïsse et Daniel de dos, empoisonnant le serpent de Babylone. La juxtaposition des deux scènes est analogue à celles représentées sur le sarcophage de Flavia Sabina du cimetière de Prétextat à Rome : Daniel est également de dos, vêtu d'un pallium plissé, devant une base d'autel à colonne torse et un arbre. Derrière lui, l'Hémorroïsse, courbée touche le vêtement du Christ (fig. 16)33.

Deux sarcophages historiés fragmentaires sont des créations narbonnaises. Le premier s'intègre dans la série des cuves « à frise continue» et à scènes de miracles (fig. 17.1), le second au thème de l'Adoratio Apostolorum (fig. 17.2) bien représenté en Aquitaine et à Toulouse, où les personnages sont généralement placés dans un cadre architectural. La composition en frise et les thèmes illustrés, l'usage abondant du trépan soulignant les boucles de la chevelure et des détails -iris, oreilles, commissure des lèvres- les apparentent aux œuvres romaines. Cependant, l'emploi d'un marbre pyrénéen, le dégagement des figures du nu de la cuve, le traitement des visages sur les deux faces, et la représentation sur le petit côté de la cuve de Quarante de Daniel dans la fosse aux lions, sous des rideaux entr'ouverts noués, plaident pour un atelier narbonnais dans lequel les sculpteurs ont « copié » ou se sont inspirés avec dextérité des modèles romains, nombreux à Narbonne ${ }^{34}$.

\section{CLIENTÈLE}

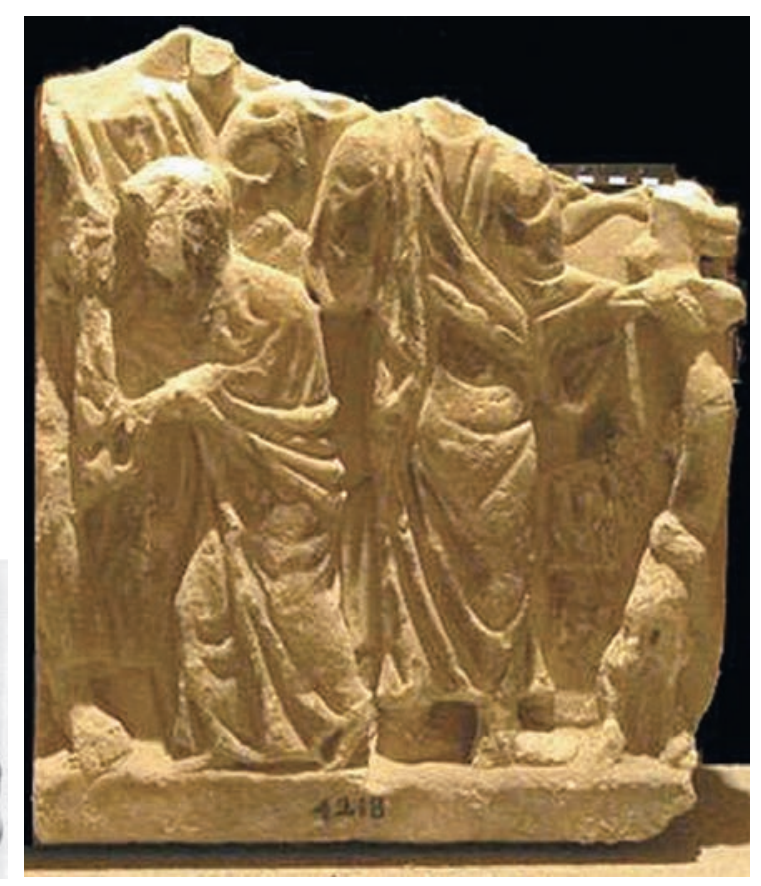

Fig. 16.1. Fragment de cuve, Guérison de l'Hémorroisse, deuxième tiers $I V^{E}$ s., marbre de Carrare, Narbonne, musée archéologique, inv. 4218. (Mérel-Brandenburg

L'aristocratie a acquis à 96\% des œuvres en marbre. La nouveauté réside dans l'adoption du sarcophage, simultanée à l'évolution des usages funéraires marqués par la dispa-

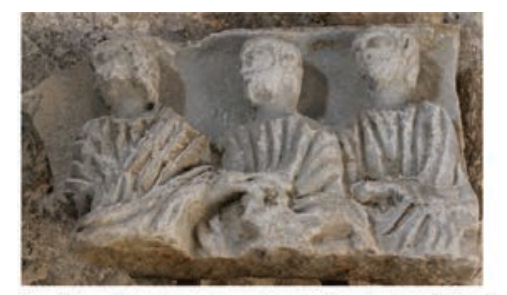

1. Narbonne, musée Lapidaire
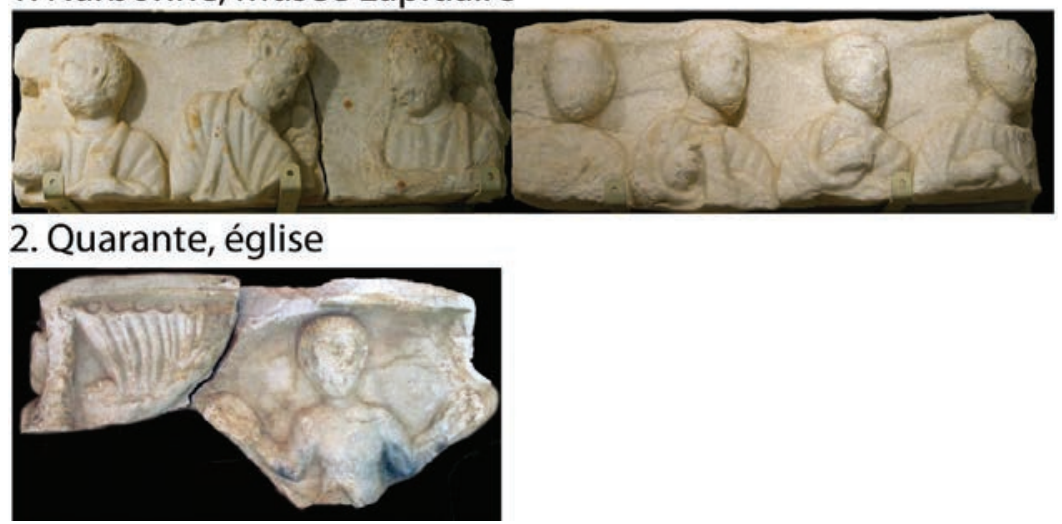

Fig. 17. 1. Narbonne, cuve de sarcophage fragmentaire, scène de miracle, marbre des Pyrénées, Narbonne, musée lapidaire. (C) Mérel-Brandenburg

Fig. 17. 2. Cuve de sarcophage, Adoratio Apostolorum, marbre des Pyrénées, Quarante, église. (C)Mérel-Brandenburg

\footnotetext{
32 B. CHRISTERN-BRIESENICK, G. BOVINI, H. BRANDENBURG, T. ULBERT (dir.), Repertorium der christlich-antiken Sarkophage, III. Frankreich, Algerien, Tunesien, Mainz am Rhein, 2003. L'auteur attribuait à un atelier narbonnais les cuves fragmentaires à frise continue sculptées d'apôtres ou de scènes des deux Testaments, s'appuyant sur le traitement des personnages aux silhouettes animées vêtus d'une tunique couverte d'un pallium, dont ils retiennent le pan ourlé et souple sur l'avant-bras gauche.

33 G. BOVINI, H. BRANDENBURG, F. W. DEICHMANN (dir.), Repertorium der christlich-antiken Sarkophage, I. Rome und Ostia, Wiesbaden, 1967, $\mathrm{n}^{\circ} 555, \mathrm{pl} .85$.

${ }^{34}$ A.-B. MÉREL-BRANDENBURG, Les sarcophages en marbre du sud de la Gaule. L'exemple du Languedoc méditerranéen: étude des matériaux, in Cartron I., Henrion F., Scuiller C. (Dir.) Les sarcophages de l’Antiquité tardive et du haut Moyen Âge : fabrication, utilisation, diffusion. Actes des XXX Journées internationales d'archéologie mérovingienne, Bordeaux, 2009, supplément Aquitania 34, 2015, p. 229-239.
} 
rition de la crémation au profit de l'inhumation. R. Turcan dénombre pour la période romaine, seulement une quinzaine de sarcophages figurés en Narbonnaise Première ${ }^{35}$, chiffre contrastant avec le nombre de sarcophages romains découverts en Arles et à Lyon. Leélite avait privilégié jusqu'alors les mausolées ou l'emploi de stèles pour signaler leur sépulture et rappeler par une épitaphe leur filiation et leur carrière aux vivants. Ce n'est pas avant les premières décennies du IV $\mathrm{IV}^{\mathrm{e}}$. que l'aristocratie, liée culturellement et politiquement à Rome a opté pour les modes romaines et acheté des sarcophages de marbre dont le message sculpté atteste l'adhésion au christianisme. Les sarcophages continuaient à exprimer une fonction sociale, ne serait-ce par le coût du matériau, et son décor.

$\mathrm{Au} \mathrm{V} \mathrm{V}^{\mathrm{e}}$ s., les commanditaires

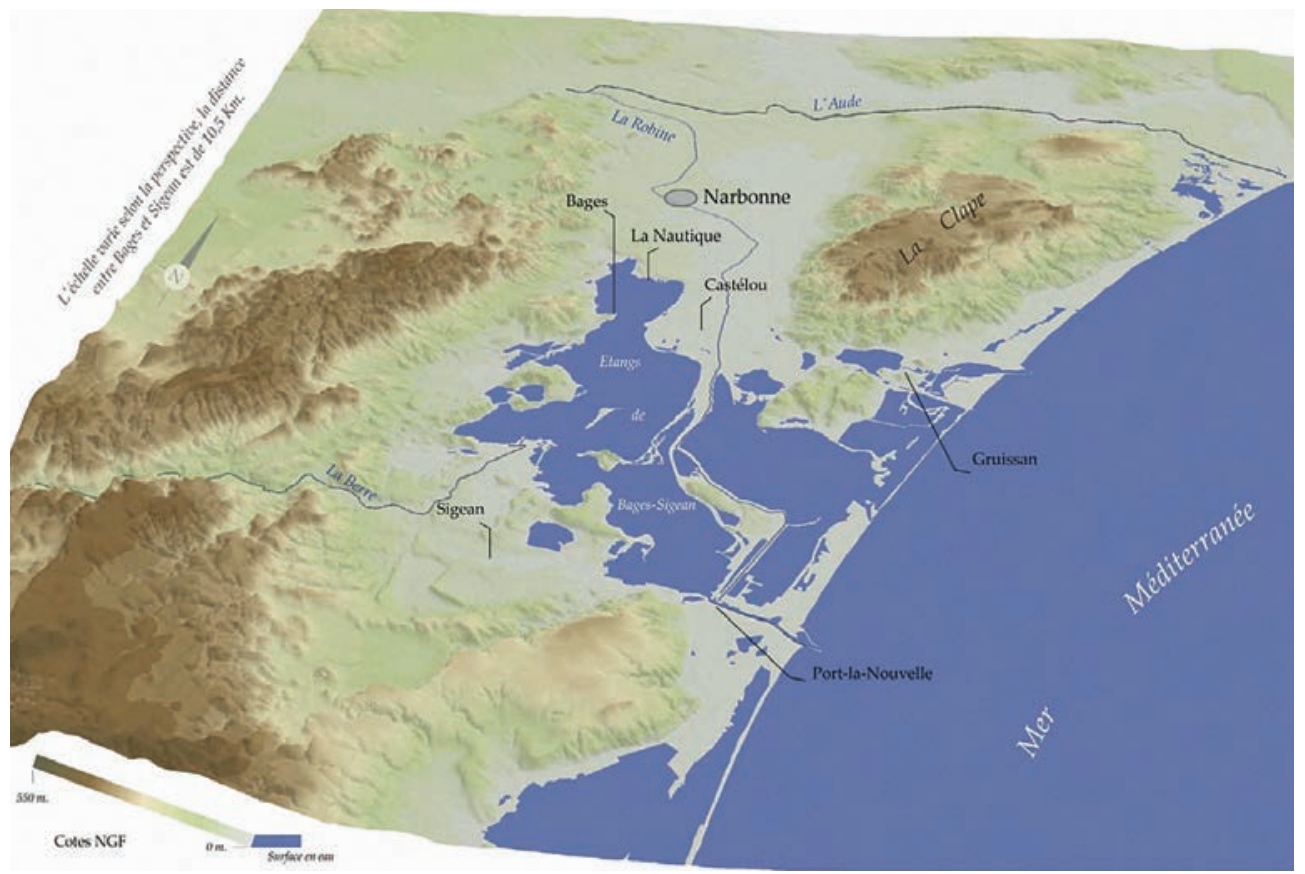

Fig. 18. Localisation de l'emplacement des installations portuaires du Ier s. au Ve s. (C) C. Sanchez ecclésiastiques ont principalement eu recours au marbre des Pyrénées, dans leurs constructions et pour le mobilier liturgique. Rusticus, évêque de Narbonne (427-461), a commandé des blocs de marbre des carrières de Saint-Béat, pour réaliser le reliquaire du Saint Sépulcre ${ }^{36}$, et les linteaux dédicatoires de la cathédrale et l'église Saint-Félix ${ }^{37}$. La cathédrale de Lyon construite par l'évêque Patiens († vers 491) et décrite par Sidoine Apollinaire était pourvue « d'un triple portique orgueilleux de ses supports en marbre d'Aquitaine $\aleph^{3}$. Vers l'an 50o, l'évêque de Limoges, Ruricius, remerciait dans une lettre l'évêque Clarus d'Eauze, de lui avoir fait parvenir des colonnes en marbre des Pyrénées avant l'hiver et ajoutait que dix autres aux dimensions plus petites lui seraient nécessaires. Leur transport ne pourrait avoir lieu après la « sainte Pâque ${ }^{39}$.

\section{CIRCUITS COMMERCIAUX}

Narbonne tient un rôle privilégiée dans l'échiquier politique et économique méditerranéen dès l'Antiquité, première fondation romaine en 118 av. J.-C., capitale de la Narbonnaise. Elle bénéficiait d'une situation géographique

exceptionnelle, par son ouverture sur la Méditerranée, ses voies de communication terrestres, la voie Domitienne menant en Italie et en Espagne, la voie d'Aquitaine vers l'Atlantique, et son fleuve, l'Atax. Commerçants, voyageurs et pèlerins y faisaient halte. L'itinéraire de Bordeaux à Jérusalem (333), l'apologie d'Ausone dans « Les villes célèbres » ${ }^{40}$, les correspondances entre Sulpice Sévère et Paulin de Nole ${ }^{41}$ de la fin du IV et du début du $\mathrm{V}^{\mathrm{e}} \mathrm{s}$., attestent le maintien de l'activité portuaire. Narbonne était, lors du séjour de Sidoine Apollinaire, aux alentours de 463-466, une ville de tradition romaine. Ce dernier en retira une forte impression qu'il consigna dans le plus bel éloge écrit de la ville antique et de son port ${ }^{42}$.

\section{Le port de Narbonne}

Les découvertes anciennes et récentes laissent entrevoir l'importance du port, deuxième port de l'Empire romain en Occident après Ostie. Il était situé dans une vaste plaine alluviale marécageuse dont l'évolution fut permanente et rapide (fig. 18). En effet, l'instabilité du fleuve, l'Aude (Atax), et du littoral ont eu une incidence majeure à l'origine de

35 R. TURCAN, Les sarcophages en Gaule romaine (des Antonins à la Tétrarchie). Essai de synthèse provisoire, Études d'archéologie sépulcrale, Paris, 2003, p. $276-277 ; 287,289,298,301$

${ }^{36}$ A.-B. MÉREL-BRANDENBURG, À propos du "sépulcre" de Narbonne (Ve siècle), in Le plaisir de l'art du Moyen Âge. Commande, production et réception de l'œuvre d'art. Mélanges en hommage à Xavier Barral i Altet, Paris, Picard, 2012, p. 84-89.

${ }^{37}$ A.-B. MÉREL-BRANDENBURG, L'architecture religieuse en Septimanie : sources épigraphiques et archéologiques, in Materiam svperabat opvs : hommage à Alain Erlande-Brandenburg, Paris, Editions de la Réunion des musées nationaux, 2006, p. 76-87.

$3^{8}$ SIDOINE APOLLINAIRE, Correspondance, II, 10, traduction A. Loyen, Les Belles Lettres, coll. « Collection des universités de France - Série latine » $\left(\mathrm{n}^{\circ}\right.$ 199), 1970.

39 R. W. MATHISEN, Ruricius of Limoges and Friends, Liverpool University Press, 1999, p. 36-39, 45, 160, 234-235 (texte et commentaire de la lettre), 253 et 261. R. BEDON, « La cité et les agglomérations des Lémovices dans les sources livresques antiques (littéraires, didactiques, administratives, cartographiques) », Siècles [Online], 33-34 | 2011

${ }^{40}$ AUSONE, Les villes célèbres, Traduction E. CORPET, C.L.F. Panckoucke, 1842 : XIII, p. 247. : «...C'est pour toi que voguent les flottes sur les eaux de la Libye et de la Sicile : et tous les vaisseaux chargés qui parcourent en tous sens les fleuves et les mers, tout ce qui navigue dans l'univers entier vient aborder à tes rives"

${ }^{41}$ PAULIN DE NOLE, Epistolae, V. 22 écrite à l'été 396 : « daigne prendre les dispositions convenables pour que l'on nous expédie le vin vieux que nous croyons avoir encore à Narbonne »; Sulpice Sévère, Dialogues, I. I. 3. ; I, 3, I : Voyage de Postumianus en 404.

${ }^{42}$ SIDOINE APOLlinAIRE, Poèmes, XXXIII, v. 37-68, Paris, Les Belles Lettres, coll. « Collection des universités de France - Série latine » (nº 161$)$, 1961. 


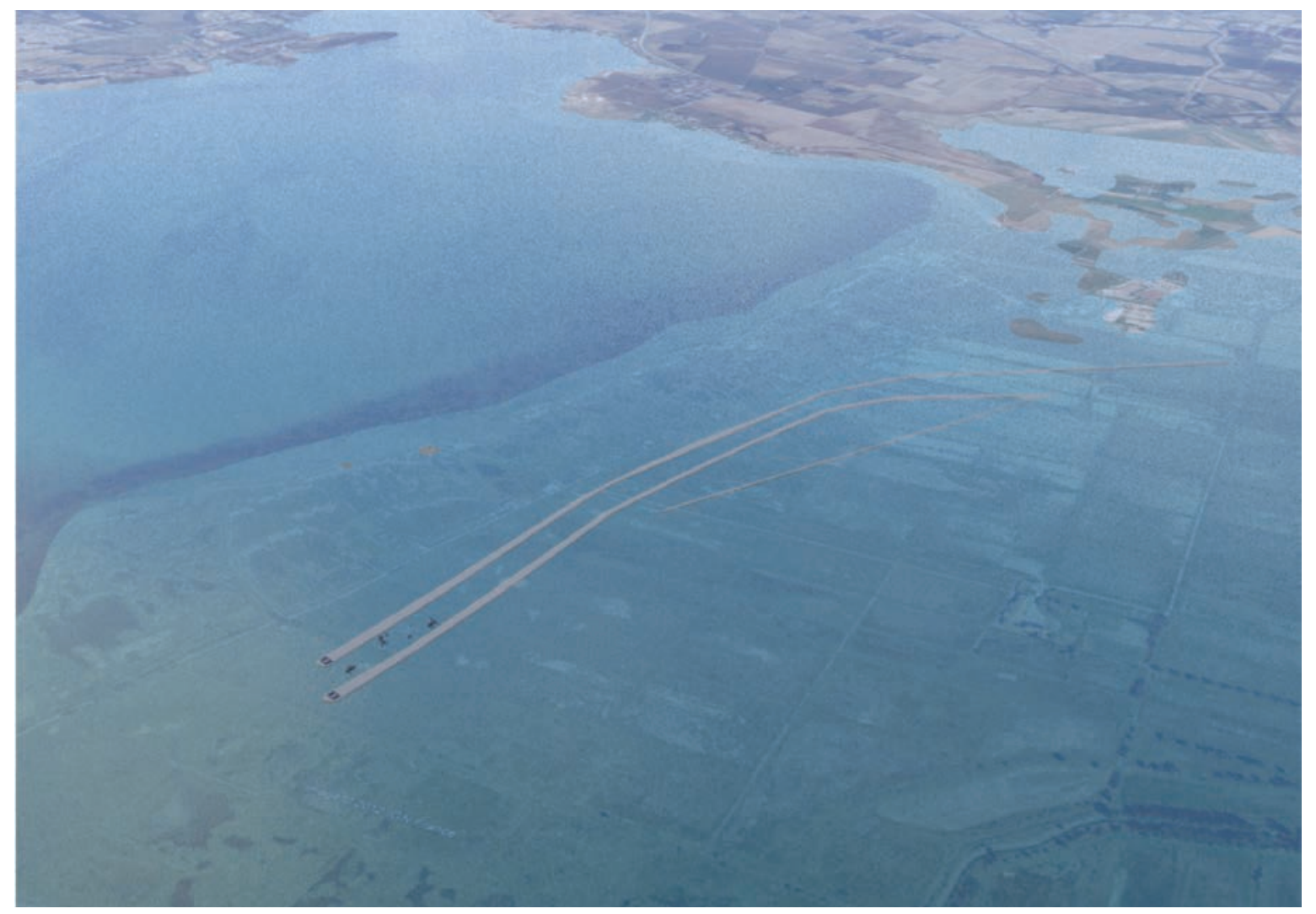

Fig. 19. Évocation de l'extrémité de l'embouchure du fleuve dans l'Antiquité au Castélou. () P. Cervellin

différentes zones de déchargements et de chargements des marchandises, étudiée dans le cadre d'un projet de recherche mené par Corinne Sanchez ${ }^{43}$.

Le port était formé de sites successivement remaniés, qui contrôlaient l'entrée des étangs narbonnais. Du site de l'île Saint-Martin à Gruissan, lieu de déchargement, à celui de Port la Nautique, lieu de transit en activité entre 30 av. J-C. et 70, Castélou/Mandirac lui succéda. Lieu de stockage et de déchargement, il a bénéficié de grands travaux pour canaliser l'embouchure du fleuve du II ${ }^{\mathrm{e}} \mathrm{au} \mathrm{V}^{\mathrm{e}} \mathrm{s}$. (fig. 19). Un canal de $50 \mathrm{~m}$ de large et au moins 3,50 $\mathrm{m}$ de profondeur et $15 \mathrm{~m}$ de large, reconnu sur près de $2 \mathrm{~km}$, a été aménagé dans la lagune grâce à l'apport de mètres cubes de matériaux et de milliers de pieux de bois renforçant les berges. À partir $\mathrm{du} \mathrm{IV}^{\mathrm{e}}$ s., de nouveaux travaux ont consolidé l'embouchure du fleuve capricieux. La digue a été reconstruite avec une coque de bateau $(3,30 \mathrm{~m}$ sur $13 \mathrm{~m})$ contenant un chargement d'amphores d'Afrique et du Sud de l'Espagne ( $2^{\mathrm{e}}$ quart $\left.\mathrm{V}^{\mathrm{e}} \mathrm{s}.\right)$ (fig. 20). Elle a été empierrée avec des éléments architectoniques, chapiteaux, colonnes en calcaire, en marbre blanc et en marbre de Turquie provenant du Capitole (fig. 21) et des sculptures appartenant à d'autres monuments qui font douter de la fidélité des descriptions faites par Ausone et Sidoine Apollinaire ${ }^{44}$.

L'activité du port et le dynamisme de Narbonne sont confortés par la découverte d'épaves ${ }^{45}$. Celle de Mateilles $\mathrm{A}^{46}$, au débouché du canal est caractérisé par l'absence de coque, mais l'homogénéité du mobilier évoque une cargaison, avec

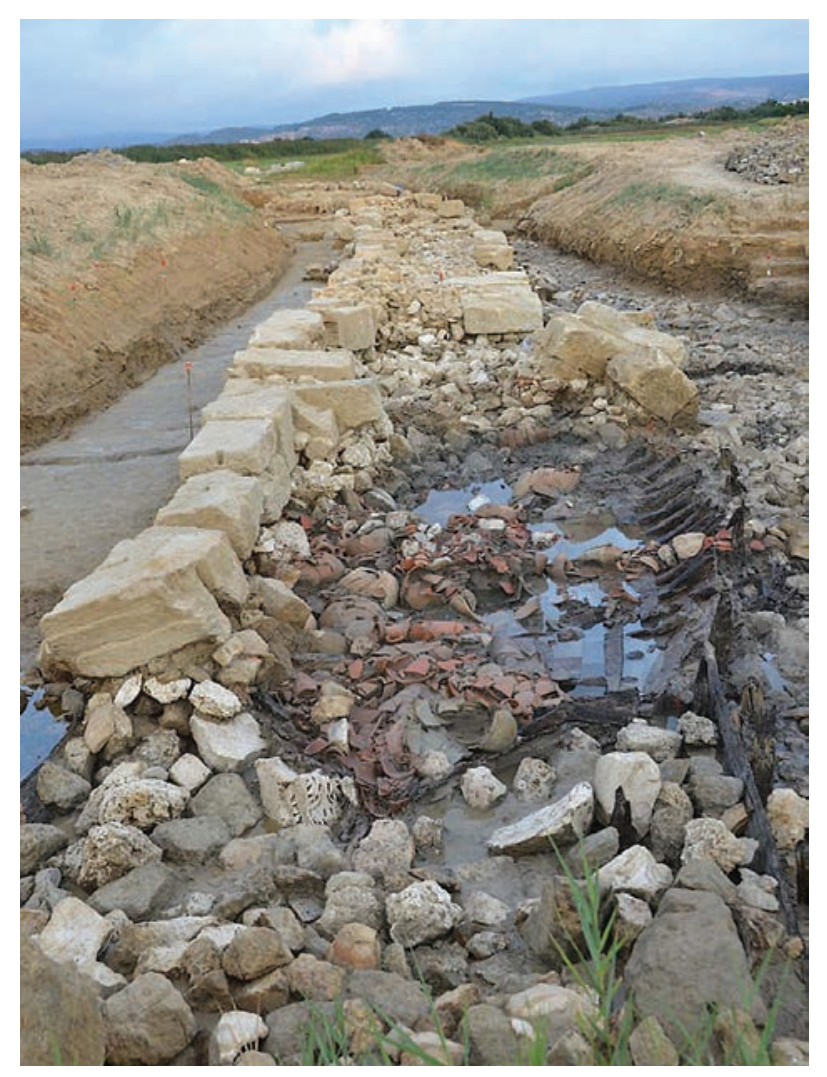

Fig. 20. Épave de l'antiquité tardive utilisée pour la construction de la digue orientale de l'ancienne embouchure du fleuve à Mandirac. () C. Sanchez

${ }_{43}$ C. SANCHEZ, C. MAUNE, G. DUPERRON, N. CARAYON, M.-P. JEZEGOU. Narbonne, un très grand port antique. in Archéologia, avril 2013, nº509, p.30-38 Région Languedoc-Roussillon et le CNRS (UMR 5140, Archéologie des Sociétés Méditerranéennes), principaux financeurs de ce programme de recherche), le ministère de la Culture (DRAC et DRASSM), l'Université Montpellier 3

${ }^{44} \mathrm{Op}$. cit notes 40 et 42

${ }^{45}$ Y. SOLIER (dir.), Les épaves de Gruissan, in Archaeonautica, 1981, vol. 3, $\mathrm{n}^{\circ} 1$ p. $26-34$.

${ }^{46}$ Y. SOLIER, Les épaves de l'étang de Mateille, Mateille A, in Y. Solier (dir.), op.cit. (n. 45) p. 176-223. 


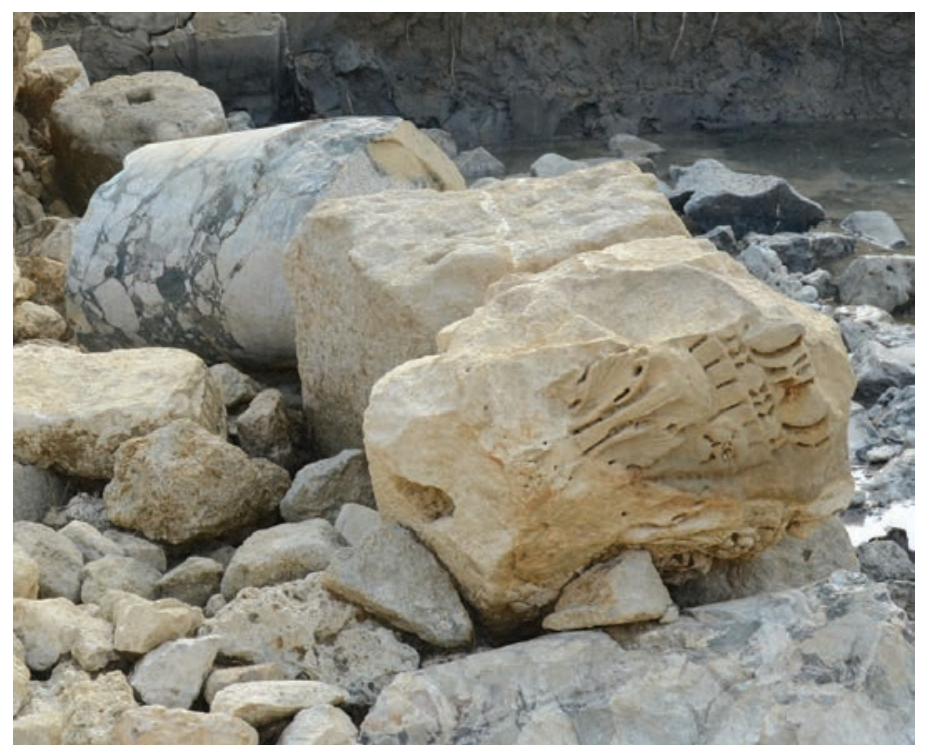

Fig. 21. Fragments de décor architectonique trouvés à Mandirac (chapiteau en calcaire, colonne en marbre de Téos). (C) C. Sanchez
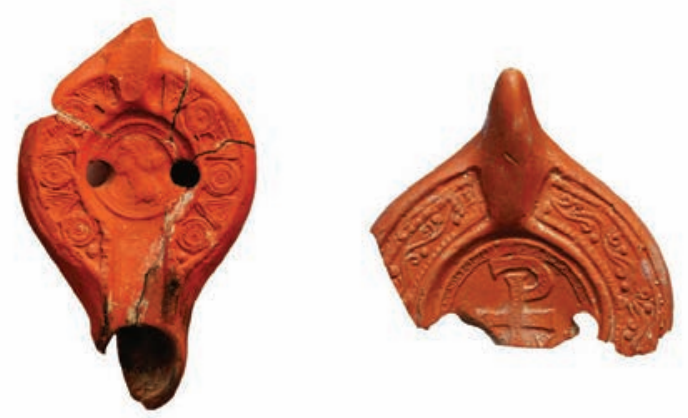

1

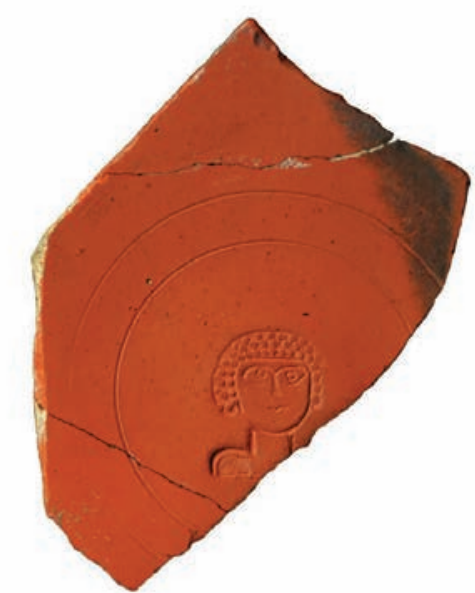

2
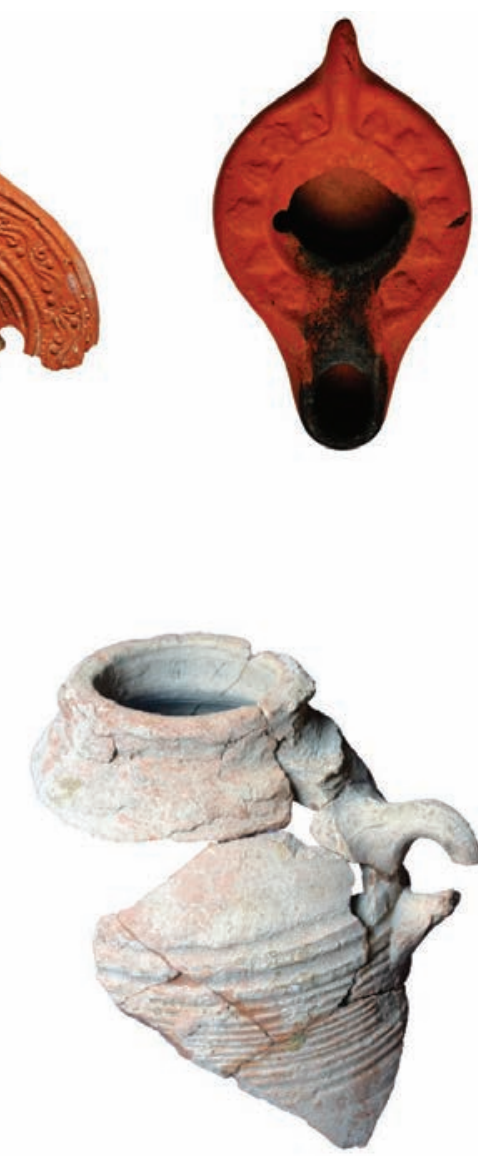

3
Fig. 22. Produits du grand commerce méditerranéen à la fin de l'Antiquité : 1-2. Lampe à huile à thème chrétien (IVe-Ve s.), sigillée claire produite en Afrique du Nord au Ve s. ; 3. Vaisselle de table; amphore à vin de Gaza () G. Duperron

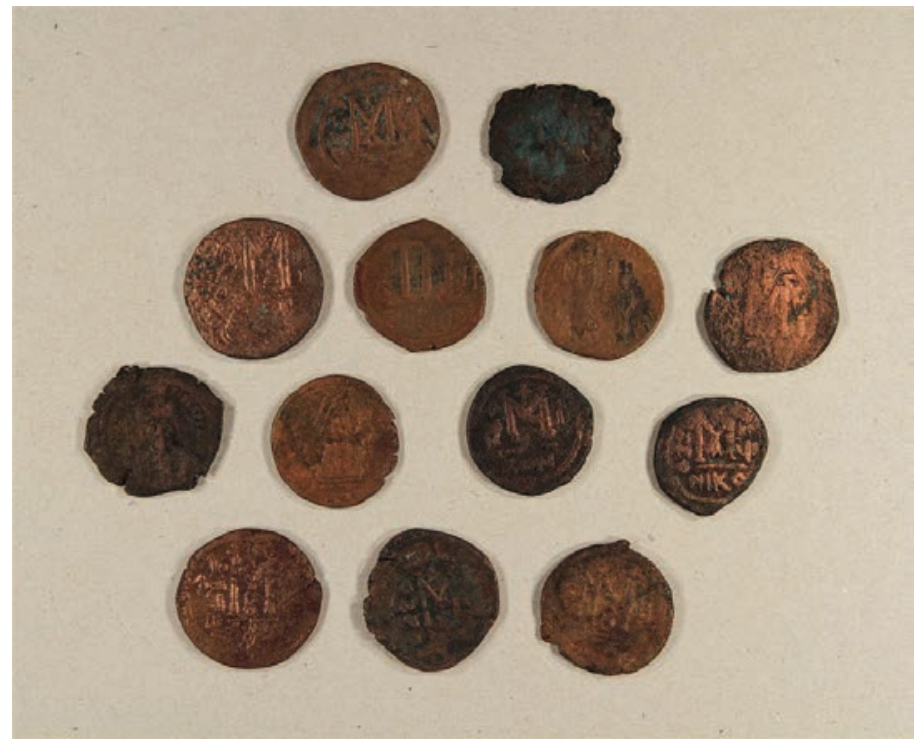

Fig. 23. Gruissan, épave de Grazel B, monnaies byzantines. (CD. Moulis

des objets en fer et alliage cuivreux, des lingots, des amphores de type byzacène, céramique commune, lampes à huile à thème chrétien $\left(\mathrm{IV}^{\mathrm{e}}-\mathrm{V}^{\mathrm{e}} \mathrm{s}\right.$.), sigillée claire $\mathrm{D}$, monnaies à $93 \%$ datées de la deuxième moitié du IVe s. (fig. 22)

Ces échanges se poursuivent au VII ${ }^{\mathrm{s}}$ s. avec l'importation de produits orientaux, comme l'attestent le gisement de Grazel B à Gruissan ${ }^{47}$. Sous le bord du chenal ont été récoltés divers objets en alliage cuivreux, -boîte cylindrique, écumoire, balance byzantine et 101 monnaies byzantines datées de la première moitié du VII ${ }^{e}$ s. (fig. 23)

Rares sont les épaves lapidaires sur la côte languedocienne. Celle de Riche Dunes 5 à Marseillan (Hérault) contenait une cargaison d'objets décoratifs à usage domestique datée du $\mathrm{II}^{\mathrm{e}} \mathrm{s}$., commande de seconde main destinée à un habitat privé : un bloc de près de 33 tonnes en marbre de Dokimeion, des plaques de marbre gris taillées en marbre de Proconnèse et en Carrare, un fût de colonnette également en Carrare retaillée à la base pour s'adapter à une base ionique attique en Pentélique et des statuettes en alliage cuivreux ${ }^{48}$. L'épave de Port-Vendres 9.4. (Pyrénées-Orientales) datée de la $1^{\text {èr }}$ moitié du $\mathrm{V}^{\mathrm{e}} \mathrm{s}$. par son chargement d'amphores de Méditerranée orientale, transportaient des matériaux de recyclage ${ }^{49}$, composés d'une centaine de blocs sculptés fragmentaires d'architectures différentes de matériau de provenances diverses - calcaires divers, marbre blanc, marbres colorés divers. Datés entre la fin du ${ }^{\text {er }}$ s. av. J.-C., et au cours du 1er s. apr. J.-C., ils provenaient de monuments détruits d'une ville de Narbonnaise, peut être utilisé comme lest pour équilibrer le navire ou en matériau de récupération pour alimenter les fours des chaufourniers.

\footnotetext{
${ }_{47}$ Y. SOLIER, L'épave byzantine de Grazel B, in Y. Solier (dir.), op. cit. (n. 46), p. 26-34

${ }^{48}$ L. LONG, Les découvertes récentes du Cap d'Agde, Leépave des Riches Dunes à Marseillan : autopsie d'un contexte archéologique sous-marin encore mystérieux, in Bronzes grecs et romains, recherches récentes - Hommage à Claude Rolley, M. Denoyelle, S. Descamps-Lequime, B. Mille et Stéphane Verger (dir.), 2012. H. BERNARD, M.-P. JÉZÉGOU, P. BLANC, B. MILLE, L'Épave Riches Dunes 5 à Marseillan(Hérault): un transport d'objets décoratifs à usage domestique au IIe siècle après J.-C., in Archaeonautica, 17, 2012, p.95-104

${ }^{49}$ G. CASTELLVI, E. NANTET, C. DESCAMPS et M. SALVAT, La corniche romaine dans l'épave Port-Vendres 9.4 ( Pyrénées - Orientales ), in Profils d'objets, TAP, éd. De Boccard, 2011, p. 201-207. Port-Vendres était un lieu de mouillage, de relâche, entre les ports de Tarraconaise, ceux de Narbonne et de Méditerranée, au cours de l'Antiquité.
} 
Les marbres méditerranéens étaient chargés sur des navires au départ de Rome et à destination de Narbonne. Ils suivaient une route longeant les côtes ligures, provençales et le Golfe du Lion en fonction des courants et vents dominants. Les navires en partance de Narbonne à destination d'Ostie avaient intérêt à longer la côte pyrénéenne en direction du Cap Creus pour rejoindre le détroit de Bonifacio et remonter le long de la côte tyrrhénienne ${ }^{50}$. Des questions inhérentes à l'organisation du commerce maritime se posent : les sarcophages étaient-ils transportés directement d'Ostie à Narbonne, les bateaux faisaient-ils escale à Fos, livrant à Arles une partie de leur cargaison, avant de poursuivre leur route vers Narbonne, ou étaient-ils entreposés à Arles, puis redistribués par voie de mer pour répondre aux commandes ? Parfois ils étaient transportés en pièces détachées : les éléments constitutifs de la cuve, soigneusement sciés, aux découpes différentes étaient ajustés à leur arrivée en atelier. Le coffre rectangulaire du sarcophage de « Lege Filiciter» à Narbonne est formé de deux demi-cuves d'inégales longueurs séparées par un joint transversal, et les petits côtés, d'un panneau encastré dans un cadre défini par l'extrémité des demi-cuves. La cuve du sarcophage « à arbres » découvert rue de la Huchette à Narbonne, de moindres dimensions, est composée de deux éléments longitudinaux assemblés en leur milieu, par des agrafes intérieures. La nature des matériaux et le lien étroit du support découpé et du décor invitent à penser que ces cuves ont été sculptées puis sciées dans un même bloc pour éviter les risques de cassure lors du transport. Ce type de sarcophage était destiné à l'exportation vers l'Italie du Nord et la Gaule méridionale (fig. 24).

Le marbre de Saint-Béat empruntait un « itinéraire continental ». Il était acheminé jusqu'à Toulouse (130 km) par la Garonne, puis à Narbonne (150 km) par la voie d'Aquitaine de Toulouse à Carcassonne (100 km), avec un transbordement sur le cours de l'Aude, lorsqu'il était navigable. En effet, Strabon au I ${ }^{\mathrm{er}} \mathrm{s}$. écrit (Géographie, IV, 1, 14), « on remonte l'Atax sur une faible distance, puis on effectue la plus grande partie du trajet par voie de terre jusqu'à la Garonne » et d'après Pomponius Mela (Chorographia, 81) «...l'Atax a un faible débit et se passe à gué. Par la suite tout en installant son cours dans un large lit, il n'est jamais navigable sauf au point où il atteint Narbonne ». De Narbonne, marbre et sarcophages étaient emmenés à Béziers (Hérault) par la voie Domitienne, ceux déposés dans le cloître de la cathédrale d'Elne (Pyrénées-Orientales), ville distante d'une centaine de $\mathrm{km}$, par cabotage jusqu'à Port-Vendres, puis par voie de terre $(18 \mathrm{~km})^{51}$. Duncan-Jones ${ }^{52}$ a tenté d'évaluer le prix du transport en se fondant sur l'Édit de Dioclétien (301), destiné à taxer les marchandises et leur transport. Ce montant

\footnotetext{
50 J. NIETO, Le commerce de cabotage et de redistribution, in Pomey P. (dir.), La navigation dans l'Antiquité, Édisud, 1997, p. 146-158

${ }^{51}$ Les sarcophages conservés dans le cloître de la cathédrale ont été considérés comme « découverts probablement à Elne » par certains auteurs (P.-A. Février, X. Barral i Altet, Topographie chrétienne des cités de la Gaule des origines au milieu du VIII e siècle, VII, Province ecclésiastique de Narbonne, De Boccard, Paris 1989, p. 42), pour d'autres auteurs, ils sont d'origine narbonnaise (Carte archéologique de la Gaule-66-Les Pyrénées-Orientales, Paris, Académie des Inscriptions et Belles-Lettres, 2007 : nº65, p. 352-357).

${ }^{52}$ R. DUNCAN-JONES, The Economy of the Roman Empire, Cambridge, 1974, p. 368.
} 
ARTISTIC EXCHANGES IN HIGH AND LATE MIDDLE AGES;

QUESTIONS OF CARTOGRAPHY 


\section{PORTI 'MONASTICI' IN CAMPANIA FRA VIII E X SECOLO.}

\section{FEDERICO MARAZZI, ALESSIA FRISETTI}

UDC: $711.453 \cdot 4: 726.7(262) " 07 / 10 "$

F. Marazzi

Original scientific paper

A. Frisetti

Manuscript received: 10. 11. 2015.

Revised manuscript accepted: 11. 03. 2016.

DOI: 10.1484/J.HAM.5.111345

Università degli Studi Suor Orsola Benincasa di Napoli LATEM (Laboratorio di Archeologia Tardoantica e Medievale)

Via Sorgente, 4 81016 Piedimonte Matese (CE), Italia

During the IX century the monastery of San Vincenzo al Volturno in Molise becames the core of a trade system of goods and products, based also on a network of harbours and docks. The most difficult geographical position of the monastery, may be suggest a possible link between the mediterranean harbours and the seaports on the Volturno river such as Ponte Latrone, "Castro Porto", Capua (source of several architectonical spolia used in the construction yard), and other settlements located next the course of Volturno river. The network organized by the community of S. Vincenzo, through the acquisition of lands and goods, is comparable with the plan setted by Montecassino. In this work we'l propose a comparison between these monasteries which try to enlarge their presence from the inland to the coastal areas. We'll try to prove these theories through the comparison of informations achieved by reading of written and cartographic sources, the analysis of architectures and the study of some precious objects (iron, silver, ivory, glass) that suggest the exsistence of relationships between the monasteries and european and non-european countries. In conclusion we suggest that this network would be the foundation of the movement of goods, products and specialized workers whith their knowledges and techonologies.

Keywords: monastic settlements; monastic harbours; medieval architecture; specialized productions.

L'affascinante tema che si propone al centro di questo simposio consente di approfondire la questione dei porti monastici campani nei secoli altomedioevali, incrociando fonti scritte ed archeologiche.

In particolare, in questa sede si tenterà di sintetizzare il quadro storico-economico e culturale della rete di porti, approdi ed infrastrutture fluviali e marittime che l'abbazia benedettina di San Vincenzo al Volturno ha realizzato a partire dal IX secolo, secondo dinamiche del tutto simili a quelle approntate dalla comunità monastica di Montecassino ${ }^{1}$.

Per comprendere l'interazione fra il sistema di porti marittimi e il capillare network di snodi fluviali "locali", dobbiamo leggere soprattutto il Chronicon Vulturnense e le fonti documentarie pertinenti a questo monastero, nonché la Chronica Monasterii Casinensis, attraverso cui è possibile seguire la dislocazione dei beni fondiari monastici posti lungo il corso del Volturno² (fig.1).

Per quanto riguarda l'alto corso del Volturno, conosciamo alcuni beni dislocati in posizioni nevralgiche: la curtis
Cicerana (donata da Grimoaldo IV nell'8103), le terre di S. Maria Oliveto (donate da Sicardo nell' $836^{4}$ ), le limitrofe terre di Maczano (ottenute tramite lascito privato ${ }^{5}$ ) ed il mulino presso la foce del fiume Sexto ${ }^{6}$. A queste proprietà che, con le terre donate dai duchi e principi beneventani Gisulfo, Arechi II e Sicardo, costituiscono un primo blocco fondiario aggiunto a valle di quello che circonda da vicino il monaste$\mathrm{ro}^{7}$, corrispondono, non a caso, alcuni beni posti sull'altra sponda del fiume nei pressi del Ponte Latrone un importante ponte romano, i cui restauri, tra l'altro, tradiscono una sua piena funzionalità ancora in epoca medioevale ${ }^{8}$ (fig.3). Questo dato suggerirebbe la continuità degli spostamenti di merci ed uomini nella zona, ulteriormente confermata dalla fondazione tra VIII e IX secolo, proprio nei pressi del ponte, di una piccola chiesa tricora che potrebbe segnalare l'ingresso al blocco centrale delle terre vulturnensi ${ }^{9}$ (fig.4).

Proseguendo verso SO, ed entrando nella Media Valle del Volturno, incontriamo le terre pertinenti alla chiesa di S. Cristina di Venafro donata nell'866, come testimonia un

${ }^{1}$ F. MARAZZI, Portus monasterii. Scali portuali monastici lungo il corso del Volturno (IX-X secolo), in Annuario ASMV, n.s. 2, 2014, pp. 201- 222; id., San Vincenzo al Volturno tra VIII e IX secolo: il percorso della grande crescita, in San Vincenzo al Volturno. Cultura, istituzioni, economia, F. MARAZZI (a cura di), Montecassino, 1996, pp. 41-92; id. The Early Medieval Alternative. Monasteries as Centres of non City-based Economic Systems in Italy between Eight and Ninth Century AD, in Nourrir les cités de la Mediterranée: Antiquité - Moyen Âge, B. MARIN - C. VIRLOUVET (dir.), Paris 2004 , pp. 739 - 768.

${ }^{2}$ Chronicon Vulturnense del Monaco Giovanni, ed. V. FEDERICI, 3 voll., Roma, 1925 - 1938 (Fonti per la Storia d'Italia, 58 - 6o); Chronica Monasteri Casinensis, "Die Cronik von Montecassino" in M.G.H. SS.XXXIV, ed. H. HOFFMANN, Hannover, 1980.

${ }^{3}$ CVI, 31 .

${ }^{4}$ CVI, 59

${ }^{5}$ CVI, 37 e 52.

${ }^{6} \mathrm{CVI}, 37$.

7 Cfr. F. MARAZZI, San Vincenzo al Volturno dal X al XII secolo. Le "molte vite" di un monastero fra poteri universali e trasformazioni geopolitiche del Mezzogiorno, (Fonti per la storia dell'Italia Medievale), Roma, 2011 ; id. San Vincenzo al Volturno. L'abbazia e il suo territorium fra VIII e XII secolo. Note per la storia insediativa dell'Alta Valle del Volturno, Montecassino 2012 (Archivio Storico di Montecassino, Studi e Documenti sul Lazio Meridionale, 15).

${ }^{8}$ Il ponte collegava i territori alifano e telesino con le terre venafrane sull'altra sponda del Volturno. Cfr. D. CAIAZZA, Ponte Latrone, in Il territorio tra Matese e Volturno, Atti I Convegno di Studi sulla storia delle foranie della Diocesi di Isernia - Venafro, Capriati a Volturno, 18 giugno 1994, Castellammare di Stabia (NA), 1997, pp.67-104.

9 Un documento del 936 (CV II, 88), in cui si parla di una disputa tra il monastero e tale Maione di Capua, cita un ponte marmoreum ed il termine "campo" che potrebbe rimandare al vicino villaggio di Campo la Fontana, toponimo a cui la tradizione erudita lega l'edificio religioso. Tuttavia, l'assenza di riferimenti più espliciti nel documento, e le citazioni di alcuni ponti marmorei dislocati in diverse zone (CV I, 66 e CV II, 169), non consentono di spingersi 


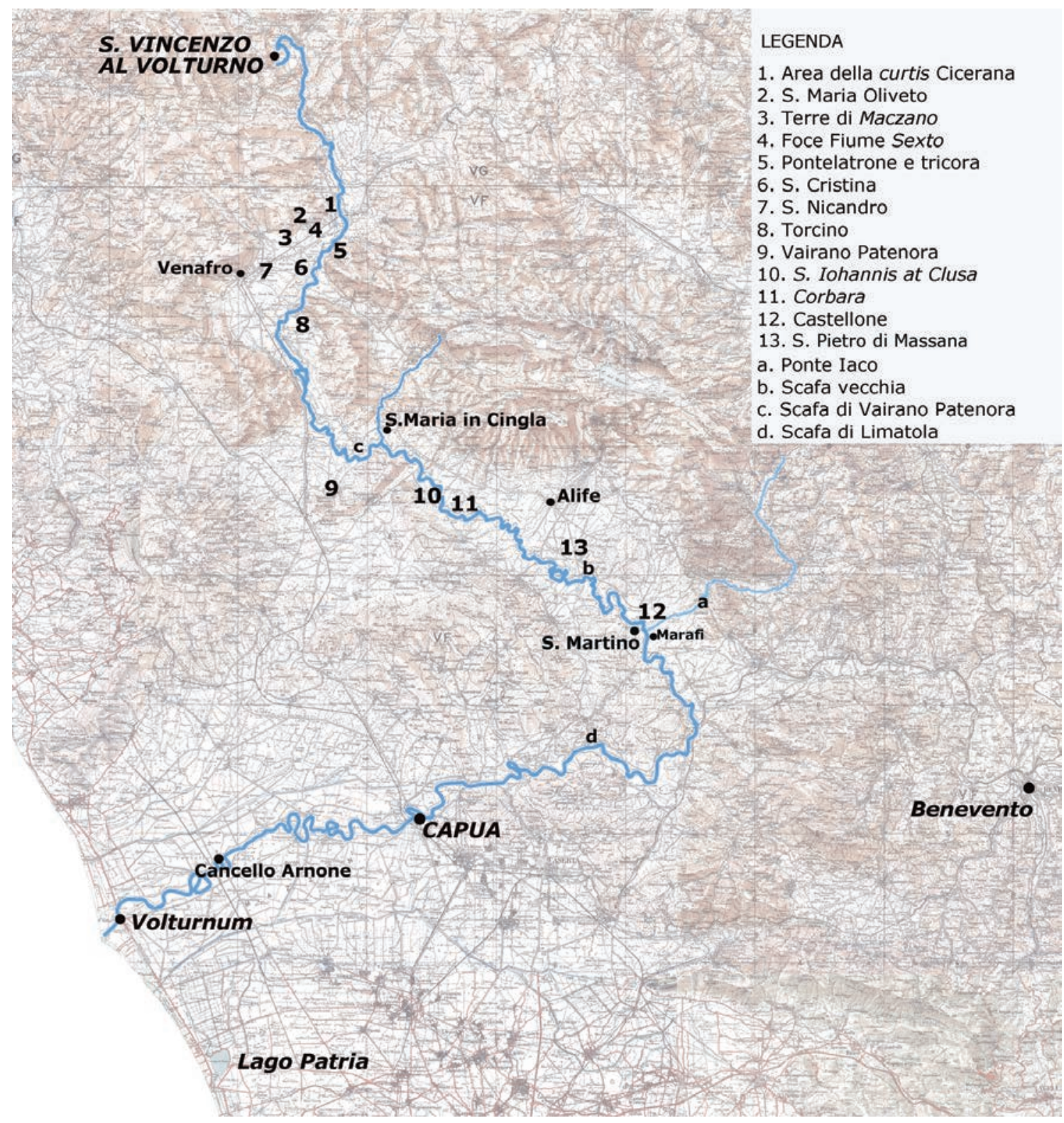

Fig. 1 Tavola d'insieme della Valle del Volturno con indicazione dei siti citati nel testo (elaborazione grafica A. Frisetti).

diploma di Ludovico II ${ }^{10}$. Questi beni nel X secolo saranno affiancati dalla curtis di Pietralata con la chiesa di S. Nican$\mathrm{dro}^{\text {"I. }}$ Sulla riva destra, invece, un importante documento dell'8o1 ricorda che il monastero entra in possesso di un gualdo in territorio di Torcino, comprendente la chiesa di $\mathrm{S}$. Pietro. I confini di queste terre giungono fino a lambire le sponde del Volturno, andando ancora una volta a completare lo scacchiere di pertinenze fondiarie già in possesso del monastero ${ }^{12}$. Nello stesso documento compare anche una "curtis de Ninfio" presso Vairano Patenora, e cioè in un'area posta poco più a Sud ed evidentemente considerata nevralgica dalle comunità monastiche dell'Italia centro meridionale (fig.1).

I documenti altomedioevali, infatti, testimoniano abbastanza chiaramente la presenza nella stessa zona, di un insediamento fortificato noto con il toponimo di "Sancto Iohanne at Clusa" (comune di Pietravairano ${ }^{13}$ ). Questo ricade in un territorio le cui terre si ritrovano suddivise fra l'abbazia beneventana di Santa Sofia (dipendente da Montecassino) già prima dell' $881^{14}$, la cella di S. Maria in Cingla $^{15} \mathrm{e}$, dalla

oltre sul piano delle ipotesi. Cfr. S. CARELLA, Architecture religieuse haut-médiévale en Italie méridionale: le diocése de Bénévent, Rome, 2011, pp.71-72; R. HODGES, S. GIBSON, A. HANASZ, Campo la Fontana: a late eight-century triconch and the Ponte Latrone at the Entrance to the territory of San Vincenzo al Volturno, in «BAR 1990», pp. 273-297.

${ }^{10} \mathrm{CVI}$, 70 .

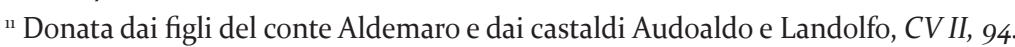

${ }^{12}$ CVI, 34 .

${ }^{13}$ A. FRISETTI, Le fortificazioni urbane di Alife e i castelli del territorio tra alto e basso Medioevo, in, "Civitas Aliphana. Alife e il suo territorio nel Medioevo". Convegno di studi, Alife 19 - 20 Gennaio 2013, F. MARAZZI (a cura di), Cerro al Volturno, 2015, pp.161-178; A. DI MURO, Roccavecchia di Pratella e il territorio alifano in età longobarda, in Atti del XVI Congresso Internazionale di Studi sull'Alto Medioevo, (Spoleto 20 - 23 Ottobre - Benevento 24 - 27 Ottobre 2002), Spoleto, 2003, pp. 1421-1427.

${ }^{14}$ CSS I, 32.

${ }^{15}$ Anch'essa dipendenza cassinese dal 961 per concessione dei conti di Teano (Gatt. Hist.I, 30). 
metà del X, l'abbazia di Montecassino. Quest'ultima possiede anche il castello di Corbara, posto nei pressi di un importante ponte di epoca romana, che potrebbe aver conservato anche nel medioevo la funzione di attraversamento del Volturno ${ }^{16}$.

Un quadro simile si presenta anche per un'altra area della media valle vulturnense, in un punto cruciale determinato dalla confluenza nel Volturno del torrente Titerno.

In questa zona le fonti ricordano la cella cassinese di S. Martino, menzionata per la prima volta in un praeceptum confirmationis di Desiderio del 761. Il documento cita la chiesa con la dicitura "Sancti Martini in Vulturnu cum portu suo ${ }^{17}$ ". L'importanza, nel lungo periodo, della cella per il monastero di Montecassino è ribadita da numerosi documenti che attestano lasciti privati in suo favore di beni, curtes e terre limitrof ${ }^{18}$, e dal diploma di conferma del principe Landenulfo di Capua del $984^{19}$. La localizzazione della cella è ancora incerta; tuttavia, tanto la tradizione orale, quanto la cartografia storica concordano nell'individuare l'area di S. Martino a Nord dell'abitato attuale di Ruviano (CE), in prossimità di un'ansa del Volturno prossima al punto di confluenza del Titerno (figg.1-2).

In particolare, una carta storica della prima metà del XIX secolo, riporta proprio in questo punto $\mathrm{i}$ toponimi di "Castellone" e "Porto Vetere" toponimo dovrebbe corrispondere il "Castello qui dicitur a lu portu" citato in un documento inedito degli anni '3o dell'XI secolo, con cui Ilario abate di S. Vincenzo concede per 29 anni a Monda (badessa del monastero beneventano di S. Adeodato) alcuni beni tra cui anche il castello sito "in finibus de comitatu

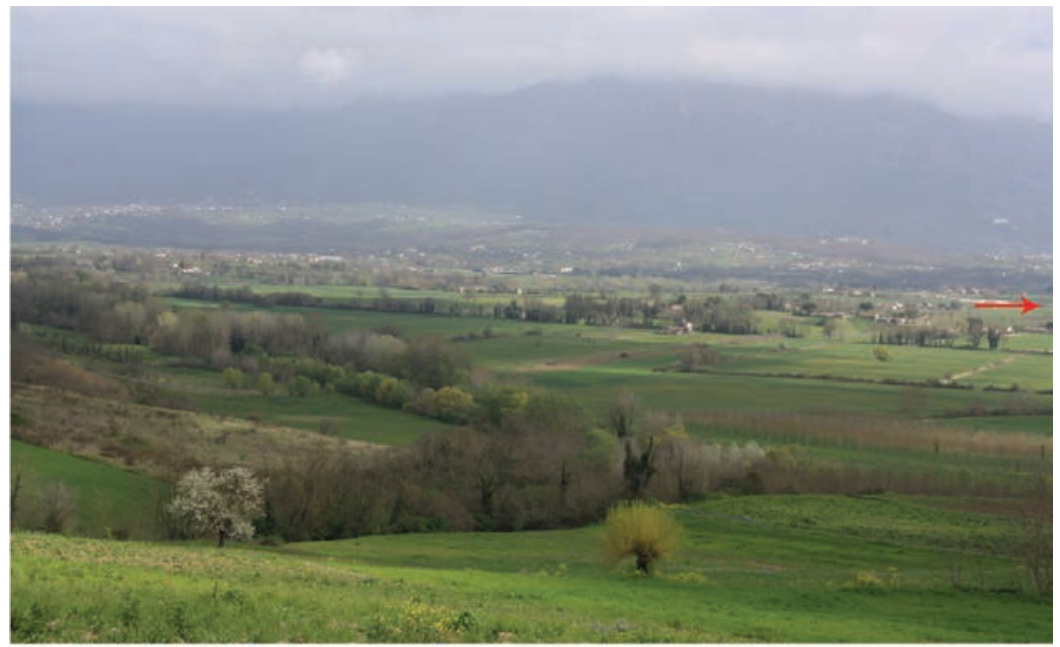
Telesino" ${ }^{21}$. Quindi ancora una volta siamo di fronte ad una piccola porzione di territorio dove si concentrano gli interessi non solo del cenobio vulturnense, ma anche di quello cassinese. Per alcuni studiosi, l'insediamento di Castellone / Castro Porto, genericamente datato in età longobarda, potrebbe rappresentare il centro amministrativo di una curtis poi successivamente dotata di apparati difensivi in virtù della sua posizione strategica, volta allo sfruttamento agricolo del territorio circostante ${ }^{22}$. In passato, comunque, le ricognizioni compiute nell'area hanno permesso di individuare le tracce di un circuito murario su tre lati della collinetta omonima, con ingresso sul lato NE affiancato da

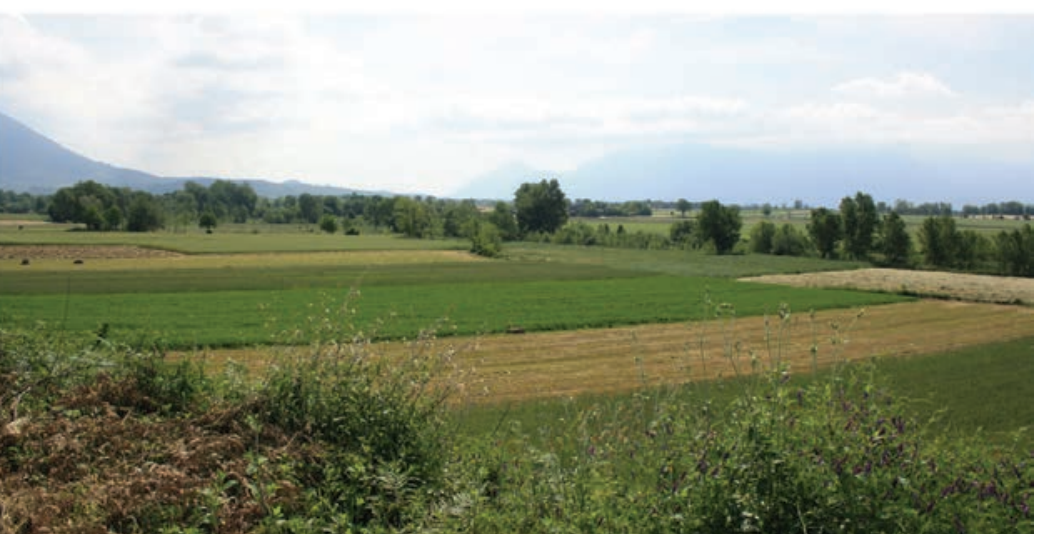

Fig. 2 In alto: panoramica dell'area di confluenza del Titerno, la freccia a destra indica la direzione verso la vicina collina del Castellone (Castro Porto); in basso: veduta panoramica dalla collina di Castellone (Faicchio, BN).

una torretta ${ }^{23}$ (fig.2). Tuttavia, si vuole ribadire in questa sede l'esigenza di abbandonare l'idea ormai superata che alla curtis di età longobardo-carolingia debba necessariamente corrispondere un'evoluzione in direzione della sua sostituzione con un insediamento fortificato, dal momento che, in questo territorio, la forma e i limiti geografici e fisici della stessa azienda curtense, con la localizzazione del suo eventuale centro demaniale, non sono ancora definibili in modo puntuale.

A completare il quadro di questo interessante distretto geografico, ricordiamo che lungo il Titerno a poche decine di metri a NE del Castellone, ritroviamo la così detta

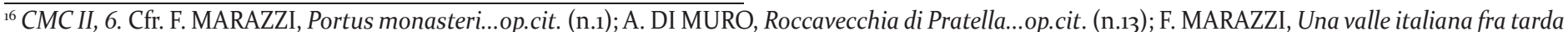
antichità e alto medioevo: il tessuto insediativo rurale della valle del Volturno (Molise-Campania) fra IV e XII secolo. Prospettive di mutamento nella "longue durée”, in Civitas Aliphana. Alife e il suo territorio nel Medioevo. Atti del Convegno. Alife 19-2o gennaio 2013, F. MARAZZI (a cura di), Cerro al Volturno, p.136.

${ }^{17}$ CDL III,1:34. A tal proposito è interessante notare come nello stesso documento, tra gli altri beni cassinesi, sia citata anche la chiesa di "Sancti Focati in Lesina cum piscaria sua”, collocata nella stessa zona del litorale pugliese dove già S. Vincenzo aveva alcuni beni ed esercitava il diritto di pesca (cfr. n.72).

${ }^{18}$ Cfr. CMC I, 18; Doc.Caiazzo V:7 e 8.

${ }^{19}$ Doc. Caiazzo V:17; CMC II, 16.

${ }^{20}$ Si tratta dei Disegni originali della Carta dei Dintorni di Napoli alla scala di 1:20.0oo, eseguiti nell'ufficio topografico dell’Ex-Regno di Napoli 1836-1840, Foglio $18^{\circ}$ N.3.

${ }^{21}$ Il testo è stato trascritto per volere dell'abate Placido in un documento del 1259, e rientra nel copioso gruppo di documenti inediti del Fondo di Vincenzo Federici, conservato presso la Scuola Speciale per Archivisti e Bibliotecari di Roma.

${ }^{22}$ Cfr. L.R. CIELO, Di alcune dipendenze dell'abbazia cistercense di S. Maria della Ferraria in territorio beneventano, in “Terra laboris felix terra”. Atti delle Prime Seconde e Terze Giornate celestiniane edite in onore della peregrinatio Celestiniana in Terra di Lavoro, D. CAIAZZA (a cura di), Quaderni CampanoSannitici X, Piedimonte Matese 2011, pp. 61-102; A. DI MURO, op.cit. (n.6) 2003; P. PEDUTO, Insediamenti longobardi del ducato di Benevento, in "Langobardia”, P. CAMMAROSANO (a cura di), Milano, 1990, pp. 307-374.

${ }^{23}$ Il lato NO sembra sprovvisto forse perché naturalmente protetto dal forte pendio che scende verso il Volturno. Cfr. G. RENDA, Il territorio di Caiatia, in Carta Archeologica e Ricerche in Campania: fascicolo 1: Comuni di Alvignano, Baia e Latina, Caiazzo, Castel Campagnano, Castel di Sasso, Dragoni, Piana di Monte Verna, Ruviano, L. QUILICI, S. QUILICI GIGLI (a cura di), Roma, 2004, pp. 239-423. 


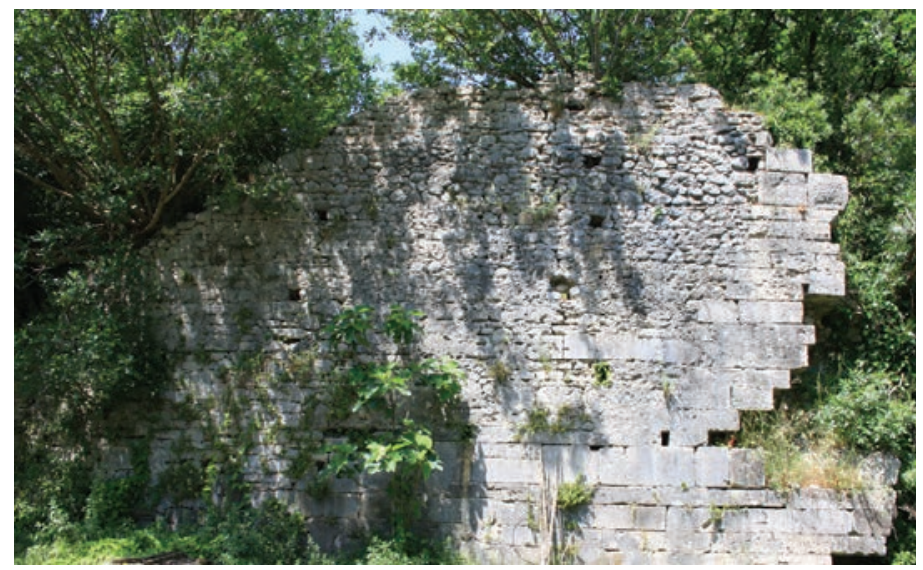

Fig. 3 Ponte Latrone (Capriati a Volturno, CE), spalla orientale del ponte con restauri medioevali.

"Scafa di S. Martino", e i resti di un antico attraversamento romano: Ponte Iaco, anche questo recante evidenti tracce di restauri medievali. Tali evidenze testimonierebbero non solo la frequentazione dell'area in tempi ben più remoti, ma anche la praticabilità del corso d'acqua ${ }^{24}$. Un'altra probabile scafa, come dimostra ancora una volta la cartografia del XIX secolo, doveva trovarsi qualche chilometro a NO, a cavallo dei territori di Alvignano ed Alife, e precisamente in un'ansa del Volturno dove si conservano numerosi toponimi che suggeriscono la presenza di un importante nodo di attraversamento fluviale ${ }^{25}$.

Questa scafa sembra aver sostituito quella localizzata più ad Ovest e ricordata appunto come "Scafa vecchia", posta nel punto in cui le acque del Torano confluiscono nel Volturno, in linea con il mulino delle Vernelle e quindi non lontano dalle selve della chiesa alifana di S. Pietro di Massana, anch'essa pertinenza di Montecassino ${ }^{26}$ (fig.1).

Questi dati, quindi, sottolineano con forza il dinamismo di un territorio a cavallo dei comuni di Alife, Ruviano, Puglianello, Faicchio e Telese che, stando ai documenti altomedioevali, doveva essere costellato di curtes e beni ceduti a vario titolo tanto a S. Vincenzo, quanto a Montecassino ${ }^{27}$.

Proseguendo verso il basso corso del fiume, ritroviamo agli inizi del IX secolo alcuni beni del monastero vulturnense in "Cancias et Insula" ossia l'odierno centro abitato di Cancello e Arnone, con la limitrofa località di "Isola" lungo la sponda sinistra del Volturno ${ }^{28}$.

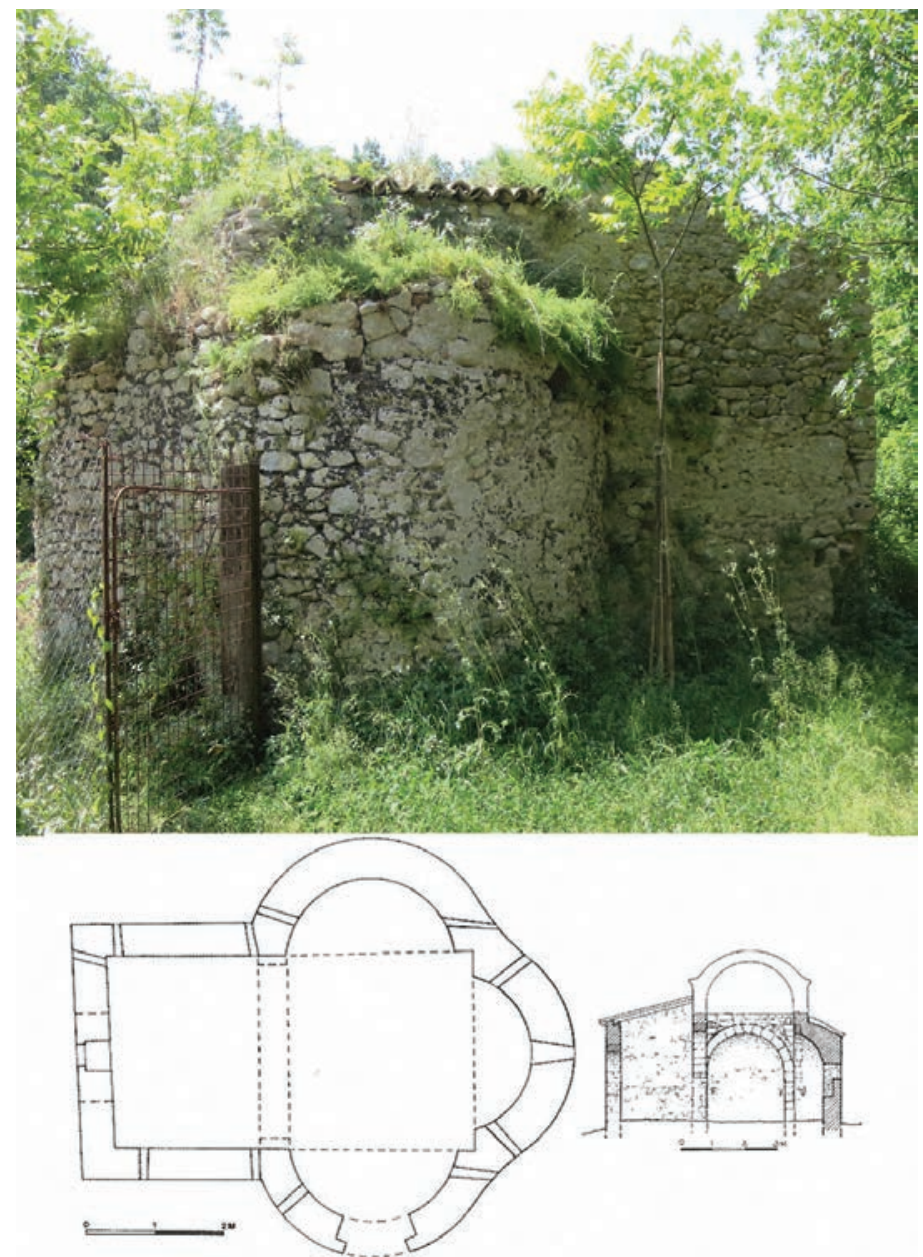

Fig. 4 Campo la Fontana, veduta della tricora presso Ponte Latrone (foto A. Frisetti) e rilievo da Hodges et alii 1990.

Ancora una volta, anche in quest'area, le due abbazie si fronteggiano per il possesso di beni e il riconoscimento di concessioni. A tal proposito ricordiamo che Montecassino riceve da Grimoaldo il porto Vulturnense, ossia Volturnum posta alla foce del fiume ${ }^{29}$. Anche S. Vincenzo ottiene beni e diritti di pesca presso il vicino lago Patria ${ }^{30}$, cui si aggiunge un "fundus in portu Neapolitano 31", cioè Napoli città e scalo marittimo di importanza cruciale per gli scambi nel Mediterraneo, dove in seguito sorgerà la chiesa dedicata a S. Vincenzo ${ }^{32}$ (fig.1).

\footnotetext{
${ }^{24}$ G. RENDA, La zona dal torrente Titerno al fiume Calore, in Carta Archeologia e Ricerche in Campania. Fascicolo 4: Comuni di Amorosi, Faicchio, Puglianello, San Salvatore Telesino, Telese Terme, L. QUILICI, S. QUILICI GIGLI (a cura di), Roma, 2010, pp. 93-274.

Tra l'altro si ricordi che sulla sponda opposta del Titerno, ma sempre in prossimità del Volturno, come testimonia anche la cartografia del XIX secolo, si conservano le tracce dell'insediamento fortificato di Marafi di impronta normanna e con annessa chiesa di S. Andrea.

${ }^{25}$ Tuttavia, le fonti scritte non forniscono dati per precisare la cronologia di frequentazione ed uso di queste scafe che, com’è noto, in alcuni casi sono state utilizzate fino agli inizi del secolo scorso. Per le scafe di Montecassino cfr. L. SERRA, Diritti di transito sulle scafe di Montecassino nel Medio Evo, in «Rivista Italiana di Ragioneria e di Economia Aziendale», Roma, 1999, pp. 646- 653.

${ }^{26}$ CMC I, 24. Anche la cartografia contemporanea riporta i toponimi di numerose scafe dislocate lungo il corso del Volturno. Tra queste ricordiamo la scafa a Sud di Torcino, quella prossima all’abbazia cistercense di S. Maria della Ferraria in comune di Vairano Patenora (Fº161 III, SE Capriati a Volturno), la scafa di Limatola ed altri attraversamenti che si collocano nel basso corso del fiume tra Grazzanise e Castel Volturno.

${ }_{27}$ Cfr. CVI, p. 240; CVI, 34

${ }^{28}$ CVI, 46 e CVI, 65, in cui compare anche un "ponte Cairusi". E' interessante notare come nella stessa zona, a partire dal 1018 fino agli inizi del XIV secolo, si concentrano alcuni beni dell'arcidiocesi di Capua. Cfr. G. BOVA (ed.) Le pergamene normanne della "Mater Ecclesia Capuana". 1091-1197, Napoli, 1996 e G. BOVA (ed.), Le pergamene longobarde della "Mater ecclesia capuana", 787-155, Napoli, 2008. Nel 1132 invece, compaiono alcuni guadi e ponti di proprietà di Montecassino (CMC IV, 97).

${ }^{29}$ CMC I, 14. Questi beni sono confermati da un precetto nell'anno 986 (CMC I, 48).

${ }^{30}$ F. MARAZZI, Conclusioni, in Il lavoro nella regola. L'approvvigionamento alimentare e il cantiere edile di San Vincenzo al Volturno fra IX e XI secolo, F. MARAZZI, A. GOBBI (a cura di), Napoli, 2007, p. 61.

${ }^{31}$ CVII, p.30.

${ }^{32}$ CV III, 44 .
} 


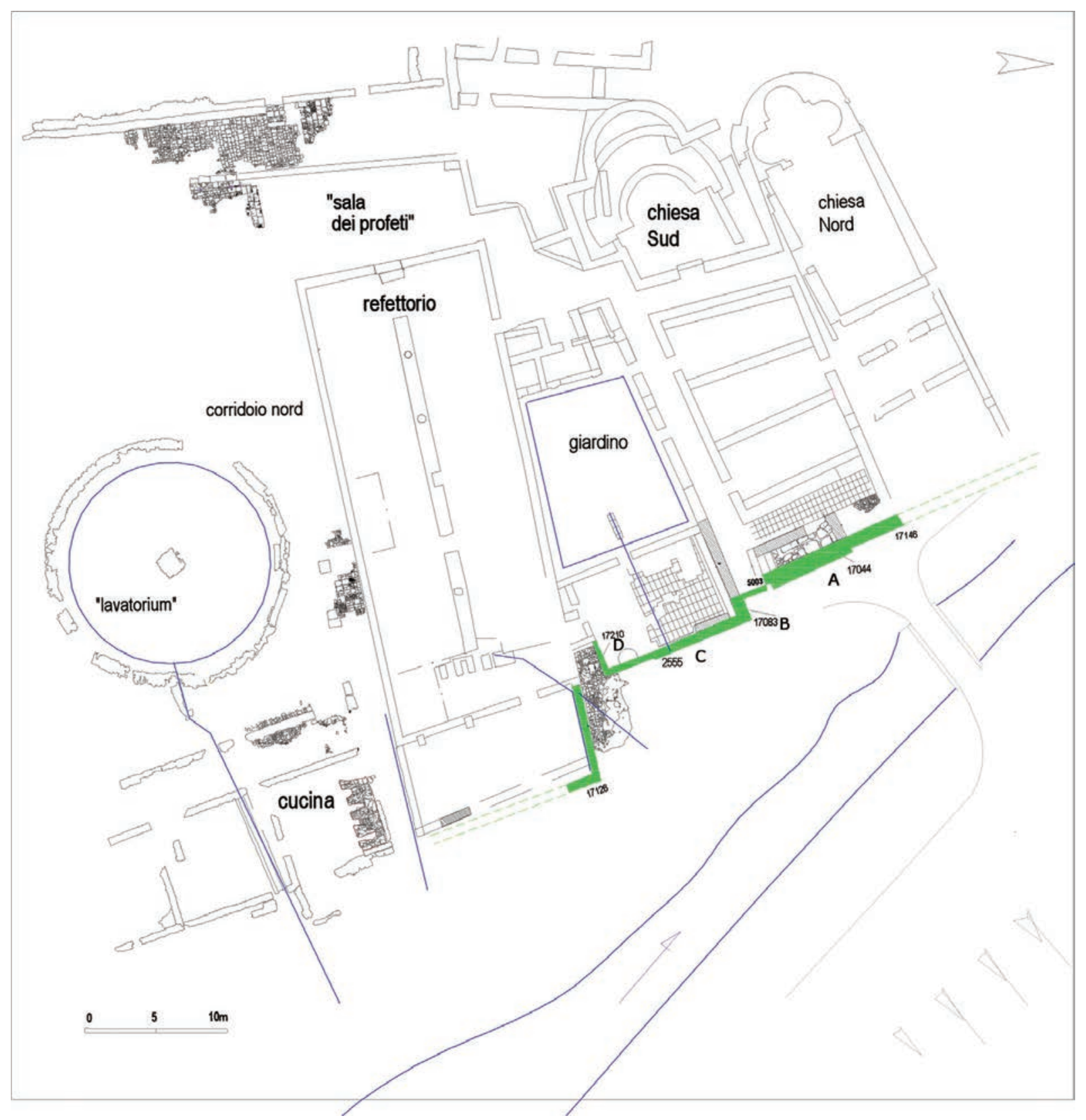

Fig.5 Planimetria degli edifici Nord di San Vincenzo al Volturno, con indicazione delle porzioni del recinto monastico sul fume (Dis. A. Frisetti).

Come si accennava prima, quindi, il monastero vulturnense organizza una rete insediativa seguendo strategie simili a quelle messe in atto dall'abbazia di Montecassino e, come suggerito per le aree dell'alto e medio corso del Volturno, anche per la zona del basso corso è possibile individuare zone di cruciale importanza poiché prossime al fiume e al litorale marittimo.

\section{DATI ARCHEOLOGICI: LE STRUTTURE}

Le indagini archeologiche dell'ultimo decennio hanno permesso di individuare anche alcune interessanti strutture e realtà insediative, a testimonianza dell'esistenza di questo articolato "network" di porti ed approdi monastici altomedioevali.
Nello specifico, gli scavi condotti nel 2006-2007 presso il sito di San Vincenzo, lungo la sponda sinistra del fiume Volturno, hanno portato alla luce alcune strutture che fungonoal contempo da supporto per il loggiato d'ingresso al monastero e da recinto protettivo per gli ambienti settentrionali dell'insediamento ${ }^{33}$. Esse, pur non costituendo parte, stricto sensu, di uno scalo fluviale vero e proprio - data la non navigabilità, in questo tratto iniziale, del corso del fiume - possono però costituire un interessante riferimento per analoghi apprestamenti per l'accesso all'acqua presenti lungo il Volturno nel medesimo periodo storico. Le strutture individuate coprono una lunghezza lineare di circa $50 \mathrm{~m}^{34}$, sviluppandosi in tre segmenti principali, secondo un andamento a dente di sega, atto a contrastare la pressione dell'acqua. Il primo tratto a Nord del recinto (Usm 17146, fig. 5, A), è costituito da un

\footnotetext{
${ }_{33}$ I dati delle indagini archeologiche sono editi in F. MARAZZI, A. LUCIANO (a cura di), Iuxta flumen Vulturnum. Gli scavi sul fronte fluviale di San Vincenzo al Volturno, Cerro al Volturno, 2015. Per quanto concerne l'analisi delle strutture cfr. A. FRISETTI, Le stratigrafie murarie e le tecniche costruttive, in Iuxta flumen Vulturnum. Gli scavi sul fronte fluviale di San Vincenzo al Volturno, F. MARAZZI, A. LUCIANO (a cura di), Cerro al Volturno, 2015, pp. 39-52. ${ }^{34} \mathrm{Il}$ recinto doveva interessare l'intero fronte orientale dell'insediamento, tuttavia non conosciamo esattamente la lunghezza del suo perimetro poiché non è stato possibile proseguire le indagini verso Sud oltre il refettorio e verso settentrione oltre la chiesa Nord.
} 


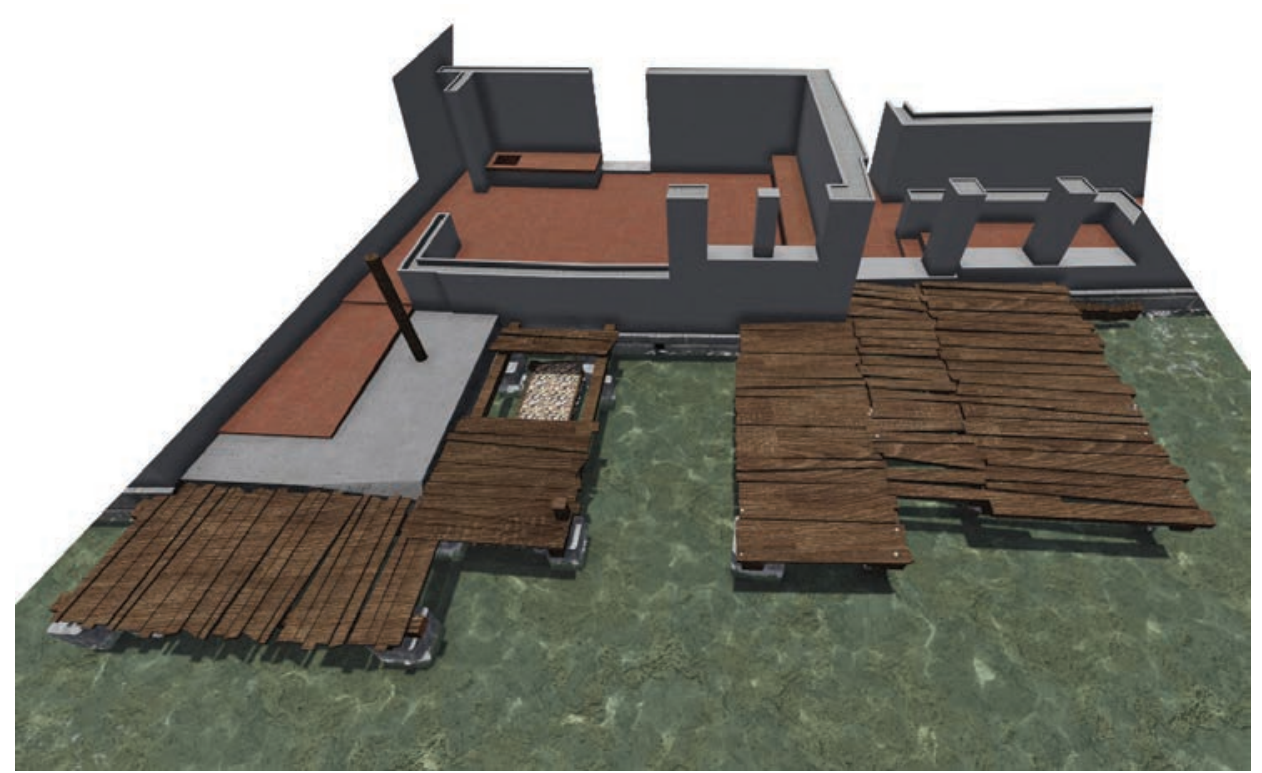

Fig. 6 Ricostruzione $3 d$ del recinto e del pontile ligneo di S. Vincenzo al Volturno (Nicodemo Abate, LATEM, UniSob).
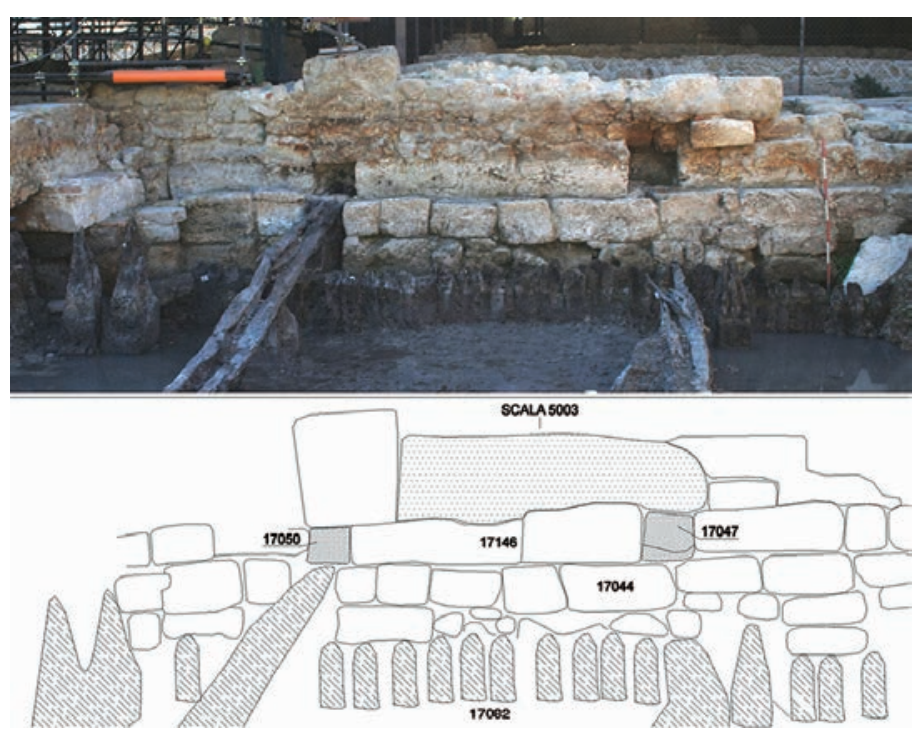

Fig.7 Il recinto monastico di S. Vincenzo al Volturno, settore $A$ (particolare e prospetto, A. Frisetti).

imponente muro di fondazione (17044), in opera pseudoquadrata in grandi blocchi di travertino (con dimensioni da $0,40 \times 0,40 \mathrm{~m}$ a $0,40 \times 0,90 \mathrm{~m}$ ). Questa struttura, parzialmente immersa nell'alveo del fiume e protetta da una fitta palificata di travi verticali (17082), costituisce il punto di appoggio del pontile, a sua volta sorretto da travi portanti a sezione quadrangolare, infisse in buche pontaie nell'alzato 17146 (fig.6). Quest'ultimo presenta un'apparecchiatura in grandi blocchi ( $1 \mathrm{mx} 0,70 \mathrm{~m})$, messi in opera in un unico filare, ed è obliterato da una seconda fase edilizia corrispondente alla spalletta orientale (5003) della scala che conduce al primo piano. Segue il collegamento tra il primo ed il secondo tratto di recinzione, rappresentato da un muretto (17083, fig..5, B) lungo circa $2 \mathrm{~m}$, con andamento Est-Ovest e costituito da due assise di grandi blocchi, che si pone quindi come un dente di sega tra le Usm 17044 e le Usm 2555 (muro Est del Refettorio degli Ospiti). In tal modo, la struttura procede a Sud in posizione più avanzata di circa $2 \mathrm{~m}$ verso l'alveo del fiume. Il secondo tratto del recinto (Usm 2555, fig.5, C) è costituito da un filare in grandi blocchi di travertino, posti su due-tre assise, con scarse inzeppature di laterizi. La porzione inferiore di questo alzato è assemblata con una serie di blocchi ed elementi di reimpiego (tra cui spicca parte di una colonna), intervallati da ampi spazi vuoti (UUSS 17119-17121-17122), per la fuoriuscita delle canalette.

L'estremità Sud di questo terzo segmento (Usm 2555) piega ad angolo retto verso l'interno creando così, un secondo dente di sega a protezione dell'angolo SE del Refettorio degli Ospiti35 (17210, fig. 5, D).

Le murature appena descritte fungono anche da supporto per una struttura di banchinaggio che doveva probabilmente seguire tutto il fronte del Volturno, dalla chiesa Nord alle cucine. Verso Sud è stato messo in luce il sistema di casseforme rettangolari anch'esse in legno, che dovevano sorreggere il piccolo molo in muratura ${ }^{36}$ (fig.6).

Il progetto ingegneristico del recinto monastico sembra ereditare il bagaglio di conoscenze tecniche tipiche del mondo romano, come dimostrerebbe l'articolazione planimetrica a dente di sega che rimanda a numerosi casi quali ad esempio il porto fluviale di Oderzo (Busana 1995). Ma ci si può spingere fino alle province romane dove l'imbarcadero de La Albufareta (Alicante, I-II d.C.), può rappresentare un buon modello di riferimento per il sistema Vulturnense ${ }^{37}$.

Per quanto riguarda la connessione degli edifici in muratura con le strutture lignee, e il sistema di palafitte quale base per le passerelle, si tratta evidentemente, ancora una volta, di sistemi costruttivi di tradizione classica, come dimostrano ad esempio le strutture rinvenute ad Aquileia ${ }^{38}$. Tuttavia un confronto più stringente dal punto di vista cronologico, è certamente rappresentato dal sistema di età altomedioevale individuato a Comacchio, che a sua volta rimanda ad altri insediamenti peninsulari39.

\footnotetext{
35 Questo setto murario, potrebbe verosimilmente rappresentare però una seconda fase edilizia, dal momento che è ben visibile l'aggancio ad Est sul recinto esterno (2555) e l'impiego di una tecnica differente, che predilige bozze e bozzette in abbondante malta.

${ }^{36}$ Per l'analisi e le ipotesi di funzionamento delle strutture lignee pertinenti le banchine fluviali, si veda A. LUCIANO, Un'interpretazione funzionale del pontile vulturnense e le sue relazioni con i contesti europei, in Iuxta flumen Vulturnum. Gli scavi sul fronte fluviale di San Vincenzo al Volturno, F. MARAZZI, A. LUCIANO (a cura di), Cerro al Volturno, 2015, pp. 53-75.

${ }^{37}$ J.R. ORTEGA PÉREZ et alii, Instalaciones portuarias del Barranco de la Albufereta (Alicante) en la antiguedad, in Le strutture dei porti e degli approdi, II seminario (Roma - Ostia 2004), A. GALLINA ZEVI, R. TURCHETTI (a cura di), Soveria Mannelli, 2004, pp. 87-111.

${ }^{38}$ F. MASELLI SCOTTI, Lo sviluppo urbano e i grandi edifici pubblici, in Aquileia. Patrimonio dell'umanità, L. FOZZATI, G. CUSCITO, F. MASELLI SCOTTI (a cura di), 2010, pp. 115-117. Ma anche le strutture individuate nel porto lagunare di Patavium, caratterizzate da palificate lignee coperte da tavole di rovere, datate al II sec. a.C. Cfr. I. MODRZEWSKA PIANETTI, F. PIANETTI, Alla ricerca del porto di Patavium, in SAGVNTVM (P.L.A.V.), 35, 2003 , pp.197-215.

39 D. CALAON, Lo scavo del Villaggio di San Francesco 1998 (COM 96). Le strutture portuali di Comacchio, in Comacchio e il suo territorio tra la tarda antichità e l'altomedioevo, S. GELICHI (a cura di), Ferrara, 2007, pp.505-530; S. GELICHI, L'isola del Vescovo. Gli scavi archeologici intorno alla cattedrale di Comacchio, Firenze, 2009. Il confronto riguarda esclusivamente le strutture e non la loro effettiva funzione. Va da sé, infatti, che mentre a Comacchio ci
} 
Anche l'analisi delle tecniche edili impiegate nella banchina vulturnense consente numerosi confronti non soltanto nell'area insulare.

L'aspetto architettonico del recinto monastico, ed in particolare l'uso della tecnica a grandi blocchi suggerisce che questa, pur con le relative varianti, sia in parte il frutto di un'imitazione di strutture classiche forse preesistenti all'insediamento monastico. Ciò è visibile soprattutto nel primo tratto del perimetro, dove la struttura di fondazione (Usm 17044), priva di tracce di sbozzatura e scalpellatura, sembra essere il risultato di un semplice riposizionamento dei blocchi preesistenti ${ }^{40}$ (figg. 5, A e 7). Un quadro differente è invece ipotizzabile
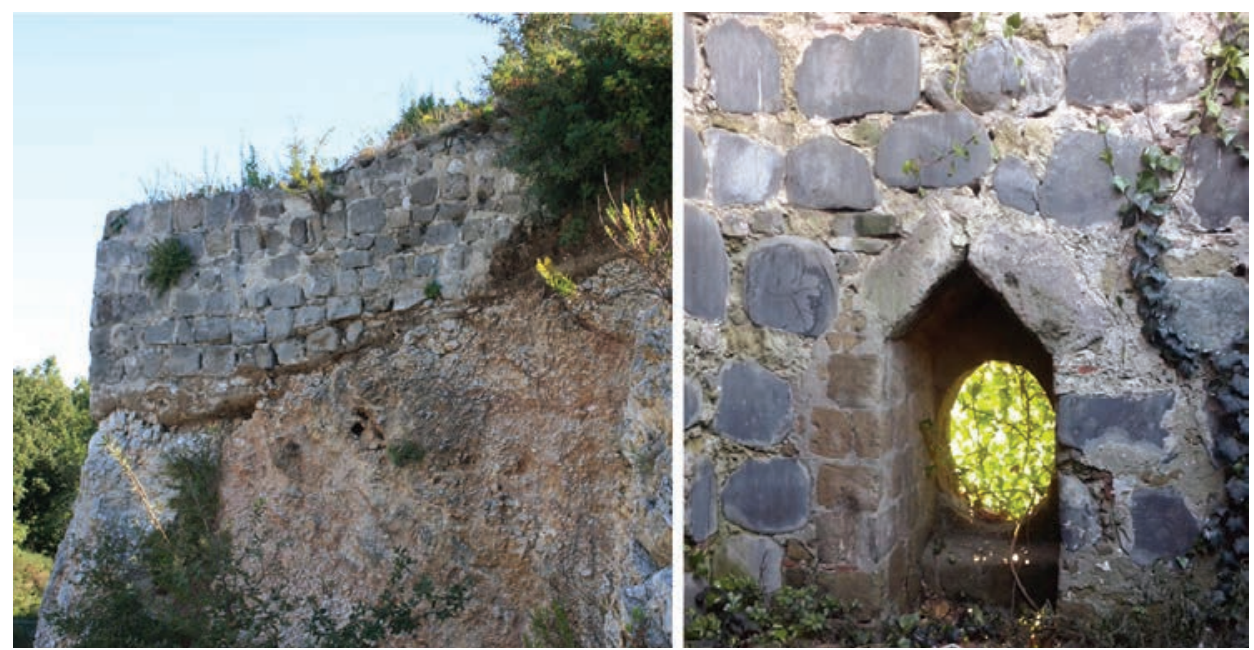

Fig. 8 A sinistra, muro di cinta di Sicopoli (collina di Triflisco-Bellona, CE); a destra, interno della torre dell'Orologio nel Castrum di Volturnum (particolare).

per gli alzati del I e II tratto, dove l'aspetto generale dei blocchi suggerisce un ciclo di rilavorazione ex-novo. In questo caso, è di conforto l'analisi di alcuni siti campani dove sono state messe in campo le stesse conoscenze ${ }^{41}$. In particolare ricordiamo il castrum di Sicopoli edificato intorno all'840 da Landolfo gastaldo di Capua per ordine di Sicone e di cui rimangono poche tracce ${ }^{2}$; e il castrum di Volturnum, alla foce dell'omonimo fiume, probabilmente voluto dal vescovo longobardo Radiperto in risposta alla minaccia saracena, ed innalzato su due arcate superstiti del ponte romano ${ }^{43}$ (fig.8).

Questi esempi, insieme a San Vincenzo, rappresentano cantieri caratterizzati da una grande accuratezza nella selezione dei materiali e della messa in opera, plausibili perché finanziati da grandi committenze che agiscono ed interagiscono in un unico grande contesto che è quello della Langobardia Minor.

Nonostante il ricorso alla tecnica a grandi blocchi mostri in Campania e in Molise un carattere episodico rispetto all'area laziale, ne è però indubbia la diffusione lungo il corso del Volturno. Data l'importanza politica e culturale di taluni insediamenti qui citati, è verosimile immaginare una certa mobilità delle maestranze specializzate, chiamate da grandi committenze a sperimentare nuove soluzioni ${ }^{4}$. Questo dato, quindi, suggerirebbe un certo legame tra i committenti di questi importanti insediamenti altomedioevali. D’altronde, non è forse un caso se, sul finire del X secolo, i Conti di Castelvolturno compaiano come testimoni in una controversia che vede protagonista proprio il cenobio vulturnense ${ }^{45}$. Ecco quindi che ancora una volta il Volturno costituisce un perfetto "trait d'union" non soltanto per scambi commerciali, ma anche per collegamenti politico-culturali che, proprio negli insediamenti di San Vincenzo, Capua e Volturnum vedono i vertici di un cruciale triangolo di comunicazione.

si trova dinanzi alle tracce di un importante sistema portuale, che fungeva da snodo tra il commercio fluviale lungo il Po e quello marittimo tra Adriatico e Mediterraneo, nel caso di S. Vincenzo, invece, le banchine erano impiegate nell'ambito di attività di minimi trasporti locali, esclusivamente finalizzate alla vita economica del monastero.

Le strutture di Comacchio, trovano confronti con molti siti dell'Europa settentrionale, ma anche con alcuni insediamenti peninsulari, quali ad esempio Torcello, dove fu rinvenuta parte di una banchina in conci quadrati, collegata a pali verticali infissi nel terreno. Cfr. M. D'AGOSTINO, L. FOZZATI, Laguna di Venezia. recenti ritrovamenti nell'isola di Torcello, in I Congresso Nazionale di Archeologia Medievale (Pisa 1997), Firenze, 2001, pp. 31-35; M. BORTOLETTO et alii, Torcello: interventi e indagini stratigrafiche in appoggio ai cantieri del magistrato delle Acque (1998-2002), in III Congresso Nazionale di Archeologia Medievale, Castello di Salerno, Complesso di S. Sofia (Salerno 2003), Firenze, 2003, pp. 228-236.

${ }^{40} \mathrm{~A}$ tal proposito rammentiamo ancora una volta il caso di Aquileia, ed in particolare le strutture fortificate di età tardo antica poste sulle banchine, e che presentano una tecnica piuttosto simile al nostro sito. Cfr. MASELLI SCOTTI op.cit. (n.38) p.124, dove però si ricorda che tali opere di IV secolo, andarono ad inficiare di fatto, la funzionalità delle banchine.

${ }^{41}$ Non si può sintetizzare in questa sede la vasta bibliografia che negli ultimi anni si è interessata all'analisi della tecnica a grandi blocchi di reimpiego, pertanto si riportano soltanto alcuni testi utili per i confronti con le nostre strutture. Tra questi ricordiamo M. CECCHELLI, Materiali e tecniche dell'edilizia paleocristiana a Roma, 2001; R. MARTA, Tecnica costruttiva a Roma nel medioevo, Roma 1989; C. CROVA, Murature medievali in opus quadratum: il Lazio meridionale e la Terra di Lavoro. Raffronti e specificità costruttive, in Tecniche costruttive dell'edilizia. Conoscere per conservare, D. FIORANI, D. ESPOSITO (a cura di), Roma, 2005, pp.105-118.

${ }^{42}$ Erch. 15 e 24; CV II:114; B. VISENTIN, La nuova Capua Longobarda. Identità etnica e coscienza civica nel Mezzogiorno Altomedievale, Roma, 2012; P. PEDUTO, Quanto rimane di Salerno e Capua longobarde (secoli VIII-IX), in I longobardi del Sud, G. ROMA (a cura di), Roma, 2010, pp. $257-278$.

${ }_{43}$ CV II, 65. L'edificio è costituito quasi interamente da blocchi di leucite, impiegati originariamente per la Via Domitiana e fatti viaggiare via mare dalle cave del Roccamonfina, forse attraversando anche un tratto del fiume Liri. Volturnum, comunque, in epoca romana è già sede di un porto marittimo, dove giungono vino ed altre merci e da cui partono i rifornimenti di grano. Ricopre un importante ruolo commerciale ancora dal IV al VI secolo, quando Teodorico lo sceglie tra i maggiori porti fornitori di grano per le Gallie. In seguito anche Procopio ricorda l'importanza del nodo di scambi costituito dal sistema "porto-ponte romano-via Domitiana" che già in epoca romana consentiva il trasporto di merci verso Capua attraverso il Volturno (Proc. Goth. VI,v.3, cfr. CRIMACO 1991, p.29 e sgg.).

${ }^{44}$ A. FRISETTI, La tecnica a grandi blocchi di reimpiego nella valle del Volturno (IX-XII secolo), in VII Congresso Nazionale di Archeologia Medievale, Lecce 9 - 12 settembre 2015, Firenze, 2015, pp. 208-213.

${ }^{45}$ CV II, 170. 


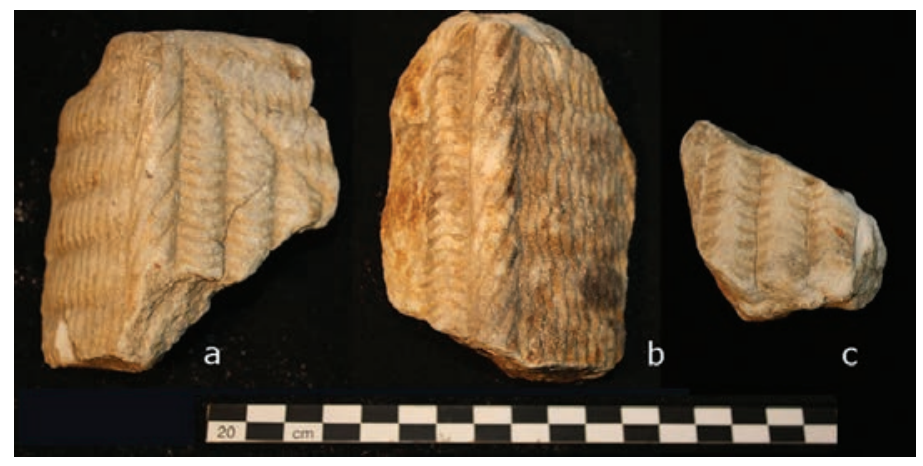

Fig. 9 Frammenti dell'urna cineraria rinvenuti a S. Vincenzo al Volturno (IS).

\section{DATI ARCHEOLOGICI: I REPERTI}

Un'ulteriore dimostrazione dell'esistenza di una rete di rapporti e traffici su medio e lungo raggio ci viene suggerita dai numerosi reperti rinvenuti in questi decenni di ricerchea S. Vincenzo. Questi possono essere essenzialmente suddivisi in due categorie: gli spolia e quelli che potremmo definire oggetti esotici, poiché chiaramente frutto di lavorazioni o di importazioni di materie prime estranee al contesto locale.

Per quanto concerne gli spolia, le indagini archeologiche nel sito monastico hanno portato alla luce cospicue quantità di materiali architettonici, quali colonne, capitelli, cornici e sectilia marmorei impiegati principalmente negli interni della Basilica Maior. Molti di questi reperti, di cui pure si è discusso in altre sedi ${ }^{46}$, oltre che provenire dalla vicina città romana di Venafro, sono certamente stati recuperati dall'antica Capua. Il canale preferenziale con Capua ed il suo territorio s'infittisce ulteriormente dal X secolo in poi, in virtù della presenza nella città di una cella monastica di $\mathrm{S}$. Vincenzo, che ospiterà i monaci dopo l'assedio saraceno dell'881. D'altronde, questo stretto contatto è noto anche grazie ad un passo del Chronicon che ricorda il diploma di Ludovico il Pio con cui si autorizza l'abate Giosuè a prelevare "columnis et diversis lapidibus" da un "templum antiquissimum" situato nella città (CV I, p. 221).

Il prelievo di spolia dall'area capuana è testimoniato anche da alcuni frammenti epigrafici "ricavati” da un'urna cineraria con decorazione a cesto di vimini (fig.9), che ha un confronto puntuale con un esemplare di I sec. d.C. proveniente da S. Tammaro (Capua ${ }^{47}$ ). Il confronto in questione, tra l'altro, trova una suggestiva conferma in un diploma del principe di Benevento Arechi II, datato al 778, in cui il prin-

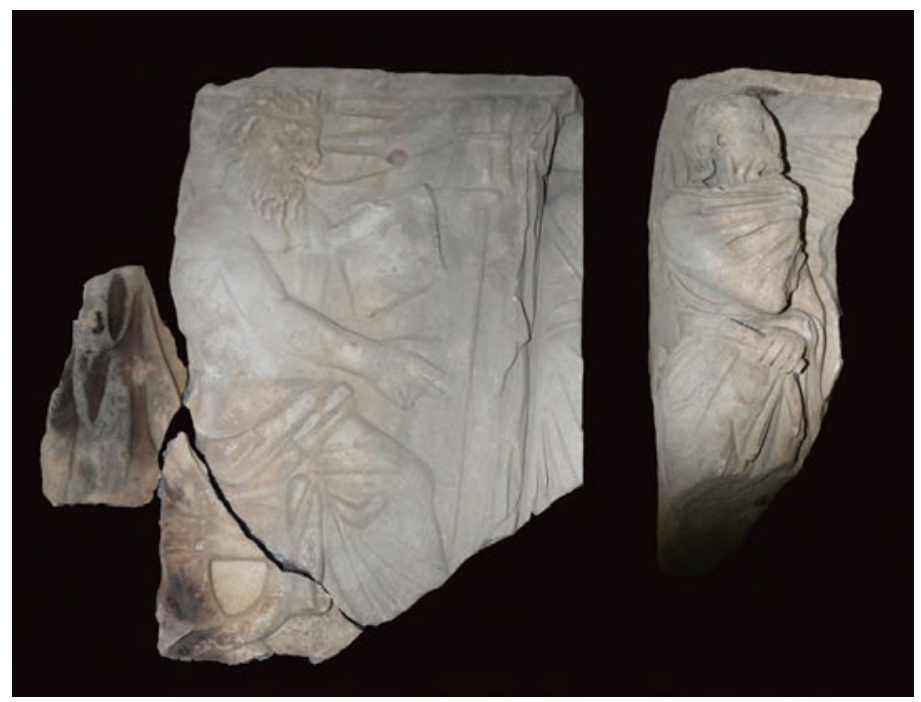

Fig. 10 Frammenti del sarcofago romano, reimpiegati nell'area del Colle della Torre alle spalle dellinsediamento di S. Vincenzo al Volturno.

cipe di Benevento cede al monastero vulturnense proprio il villaggio di S. Tammaro con tutti i servi, i beni mobili ed immobili ${ }^{48}$.

Questi spolia, insieme al famoso vaso bacchico che doveva ornare il giardino porticato ${ }^{49}$, attestano quindi un assiduo contatto fra San Vincenzo e la zona interna e costiera della Campania, probabilmente basato proprio sul sistema di collegamento fluviale.

Una prova dei contatti che il monastero intesse con aree extra regionali, è fornita, invece, dal rinvenimento nei recenti scavi compiuti sulla sommità del Colle della Torre di un'ampia porzione di sarcofago pertinente al gruppo dei "Musensarkophage" realizzati nelle botteghe romane dalla metà del II alla fine del IV secolo d.C50 (fig.10). Questo esemplare (costituito da 4 frammenti che permettono di ricostruire soltanto una minima parte dell'oggetto), conserva sul fianco sinistro parte di una figura maschile in habitus filosofico e, all'estremità sinistra del fronte una parziale figura femminile ammantata. La posa della figura femminile, la sua posizione all'estremità sinistra della scena e i relativi attributi, farebbero propendere per un'identificazione della stessa con Polimnia, una delle nove muse della mitologia greca $^{51}$. Un buon confronto è offerto dal così detto sarcofago Agliè (datato fra $230-240$ d.C. ${ }^{2}$ ), e in modo più stringente da un esemplare proveniente da Castellammare di Stabia datato al 250 d.C. (Castellammare W 2453).

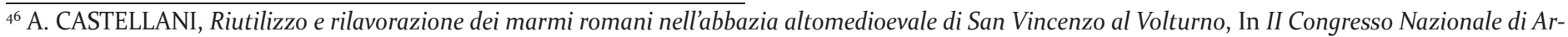
cheologia Medievale (congresso Brescia, 2000), Firenze, 2000, pp. 304-308; L. CATALANO, La produzione scultorea medievale nell'abbazia di S. Vincenzo al Volturno, Napoli, 2008.

${ }^{47}$ Per l'analisi dell'esemplare cfr. S. TUCCINARDI, Scheda VIII.59, in "Augusto e la Campania: Da Ottaviano a Divo Augusto.14-2014 d.C." Catalogo della mostra, Museo Archeologico Nazionale di Napoli (19 Dicembre 2014 - 4 Maggio 2015), T.E CINQUANTAQUATTRO, C. CAPALDI, V. SAMPAOLO (a cura di), Milano 2014, p.96.

${ }^{48}$ CVI, 22.

${ }^{49}$ Datato alla seconda metà del II sec.d.C. Cfr. A. CASTELLANI, Riutilizzo e rilavorazione...op.cit. (n.46), 2001. J. MITCHELL, A. CLARIDGE, Roman and early medieval sculpture, in San Vincenzo al Volturno 3, J. MITCHELL, I.L. HANSEN (ed.), Spoleto, 2001, pp.147-151, figg. 5:37/5:39.

$5^{\circ}$ A. TEATINI, Un sarcofago con le Muse, Apollo e Atena dalla Sardegna, in "Annali della Facoltà di Lettere e Filosofia dell'Università di Sassari", vol. 1, p. 384; L. PADUANO FAEDO, I sarcofagi romani con muse, in Aufstieg und Niedergang der römischen Welt, II, 12.2, Berlin, 1981, p. 78; M. WEGNER, Die Musensarkophage, Berlin. 1966.

${ }^{51}$ M.E. MICHELI, Sarcofago con muse (inv.n.125353), Scheda, in Museo Nazionale Romano, I/8, Parte I, Roma, 1985, pp. 31-34.

${ }^{52}$ L. PADUANO FAEDO I sarcofagi romani... op.cit. (n.50), 1981, pp. 80-84; F. MIELE, Arte e cultura itineranti. Considerazioni sul sarcofago da San Nicolò Gerrei (Cagliari) con Apollo, Atena e le Muse, in «HABIS» 40, pp.215-227 2009; A. TEATINI, Un sarcogafo...op.cit. (n.50), 2009.

53 Secondo la classificazione in M. WEGNER, Die Musensarkophage, Berlin, 1966.
} 
La suggestione di un sistema di scambi e contatti ad ampio raggio, che potremmo definire "euro-mediterraneo" è invece suggerita dall'analisi di reperti cosi detti "esotici" ${ }^{54}$. Tra questi ricordiamo il set di guarnizioni per cintura porta-spada, già analizzato in altre sedi, e costituito da elementi in ferro con decorazione ad agemina in argento ed inserti in rame ${ }^{55}$ (fig.11, a). Il set trova confronti con altri elementi simili prodotti in Italia settentrionale dopo il $774 \mathrm{e}$ anche in zone limitrofe al regno franco come la Boemia, la Moravia e la Croazia ${ }^{56}$. In particolare, l'elemento ovale (con decorazioni ad "albero della vita") ha confronti nell'area comascolombarda (es. S. Salvatore di Brescia, VIII-IX ${ }^{57}$ ). L'altro elemento, con forma trilobata, ha invece una decorazione ben conosciuta nel mondo franco, interpretabile forse come risultato della diffusione di un modello nato proprio in Italia settentrionale, dalla commistione di elementi culturali longobardi e bizantini riletti in chiave carolingia ${ }^{58}$.

L'altro reperto è un disco in rame ( $\mathrm{RN} 6162)$, forse pertinente ad un contenitore metallico di uso liturgico, ornato da fili saldati, anch'essi in rame, con castone centrale e decorazione a giralis9 (fig.11, b). Il motivo, poco presente sul territorio peninsulare, trova confronti ad

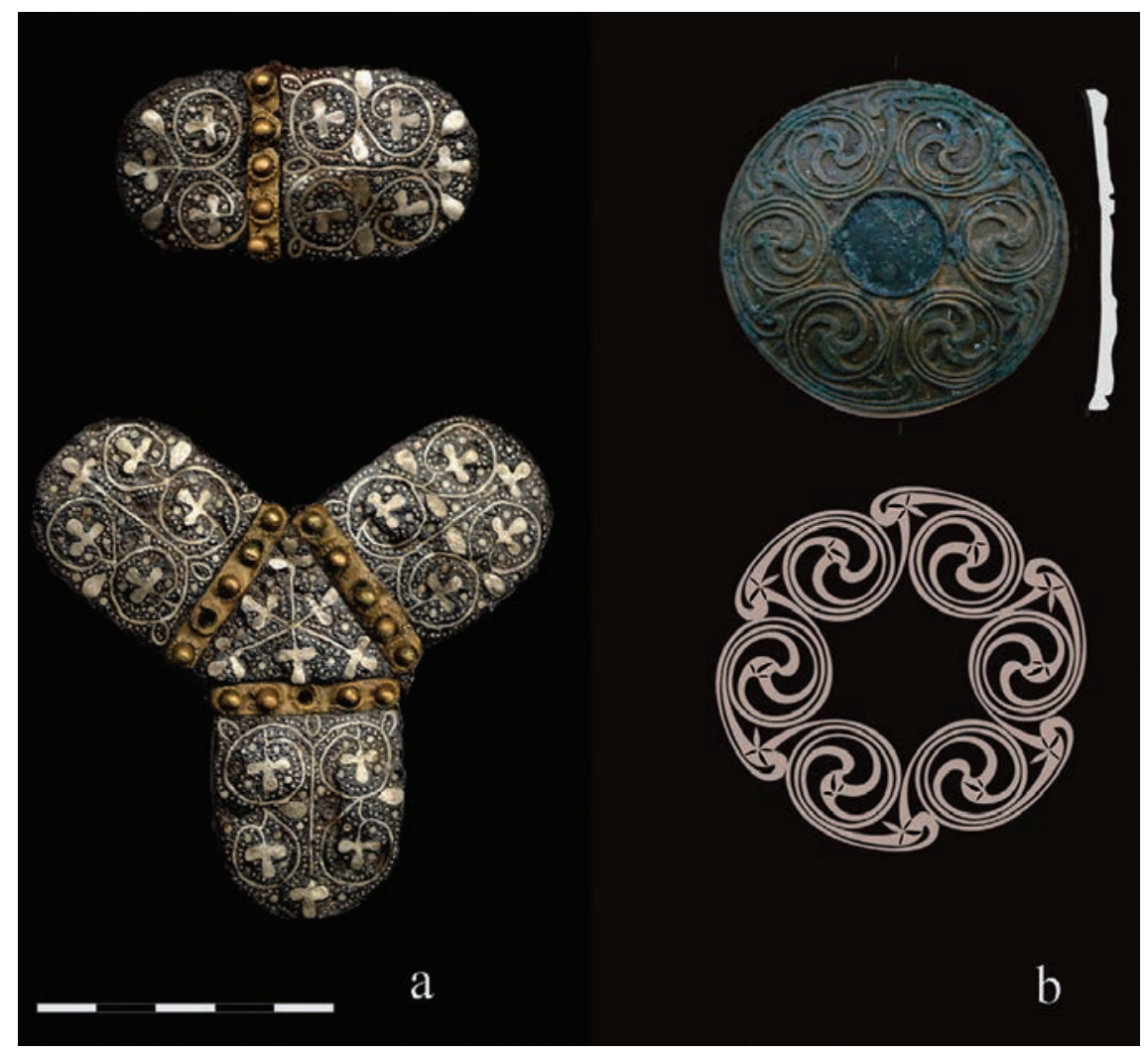

Fig. 11 Set di guarnizioni per cintura - porta spada e medaglione dagli scavi di S. Vincenzo al V. (studio di N. Abate).

esempio nel reliquiario in avorio e bronzo dal monastero di Gandersheim (VIII-IX sec. ${ }^{60}$ ), ma anche in alcune pagine dell'evangelario di Bobbio, e numerosi oggetti provenienti dall'Europa settentrionale ${ }^{61}$, mentre è del tutto assente nel cenobio molisano. Al momento attuale sono diverse le ipotesi che spiegherebbero la presenza di questi oggetti nell'insediamento vulturnense. Questi, infatti, potrebbero avere origine alloctona, ed aver raggiunto il monastero tramite viaggiatori provenienti dalle aree transalpine dell'Impero carolingio; ovvero potrebbero essere repliche di manufatti nordeuropei, eseguite in loco da maestranze specializzate itineranti. In entrambi i casi è forte la suggestione di contatti

culturali (se non pure commerciali) tra il monastero e le aree dell'Europa settentrionale.

Infine, l'ipotesi di un canale extraeuropeo, a disposizione della comunità vulturnense, proiettato anche verso aree orientali, è suggerita dalla scelta di alcune materie prime e/o dalla tecnica impiegata nell'esecuzione di altri noti reperti. Il primo di questi è rappresentato dalla testina eburnea di santo databile alla I metà del IX secolo (fig.12, a). L'oggetto, che doveva probabilmente costituire parte del rivestimento di una capsella, risulta di finissima manifattura (date anche le esigue dimensioni), ed è stato realizzato su una zanna di ippopotamo con applicazione di perline vitree per gli occhi ${ }^{62}$.

${ }_{54}$ N. ABATE, Circolazione di merci, artisti ed idee nell'alto Medioevo: il caso del monastero di San Vincenzo al Volturno (IS), in VII Congresso Nazionale di Archeologia Medievale (Lecce 9 -12 settembre 2015), P. ARHUR, M. L. IMPERIALE (a cura di), Firenze, 2015, pp.213-217.

55 J. MITCHELL, I.L HANSEN, San Vincenzo al Volturno...op.cit. (n.49), 2001, Vol.I pp. 393-412; Vol.II, pp. 337-344.

${ }^{56}$ A. MILOŠEVIĆ, Oggetti preziosi, segni distintivi carolingi della Croazia. I Tesori della Croazia medievale, in L'Adriatico dalla tarda antichità all'età carolingia, G.P. BROGIOLO, P. DELOGU (a cura di), Firenze, 2005, pp. 245-270.

${ }^{57}$ P. M. DE MARCHI, Analisi e riflessioni sulla produzione lapidea nel territorio varesino. Note sulla decorazione architettonica e funeraria nell'alto medioevo, in Castel Seprio e la giudicaria. Cinquant'anni di studi: resoconti e nuove proposte, D. LIMONATA, A. BERTONI, P.M. DE MARCHI, Milano, 2003, pp. $100-106$.

${ }^{58}$ In generale il set, inquadrabile nel IX secolo, trova confronti con corredi dell'Europa centrale e settentrionale (Boemia, Renania-Vestfalia, penisola scandinava, Paesi Bassi e Sud-Est del Regno Unito). Alcuni esempi di set simili sono stati rinvenuti a Kolìn (C. STIEGEMANN, M. WEMHOFF, 799 Kunst und Kultur der Karolingerzeit. Karl der Große und Papst Leo III, in Paderborn Katalog Der Ausstellung Paderborn, 2 Vol. C. STIEGEMANN, M. WEMHOFF (ed.), Mainz, 1999, pp. 754-755), a Ballhorn (G. EGGENSTEIN, Balhorn in der Merovinger-und Karolingerzeit. Bebachtungen zu Infrastruktur, Wirtschaft und Soziologischen Indikatoren, in Il cammino di Carlo Magno, F. MARAZZI, S. GAI (a cura di), Napoli, 2005, pp. 57-78), a Roskilde (R.A. SMITH, A guide to the Anglo-Aaxon and Foreign Teutonic Antiquites in the Department of British and Medieval Antiquites, London, 1923), a Östra Påboda (H. ARBMAN, Schweden und das Karolingiske Reich. Studien zu den Handelsverbindungen des 9. Jahrunderts, Stockholm, 1937, fig. 147; C. STIEGEMANN, M. WEMHOFF, 799 Kunst und...op.cit. (n.57), 1999, pp. 753-754), a Maastricht-Amby (B. AGER, Fragment of a Carolingian trefoil mount from a swordbelt, found at Maastricht-Amby, in Jaarboek '98-'99. Publications de la Societé Historique et Archéologique dans le Limbourg, Deel 134-135, Maastricht, 2000, pp. 427-436).

${ }^{59}$ Motivo decorativo molto conosciuto nella toreutica, nell'oreficeria e nella scultura del Nord-Europa tra V e IX secolo. Cfr. N. ABATE, Circolazione di merci...op.cit. (n.54). 2015. Questi stessi motivi decorativi, tipici del paganesimo celtico, sono poi ripresi dai monaci amanuensi ed introdotti negli evangelari. ${ }^{60}$ C. STIEGEMANN, M. WEMHOFF, 799 Kunst und...op.cit. (n.58), 1999.

${ }^{61}$ In particolare gli "escutcheon", ossia piccoli dischi da applicare su contenitori ed oggetti di diversa funzione.

${ }^{62}$ F. MARAZZI, San Vincenzo al Volturno. La vita quotidiana di un monastero altomedioevale vista attraverso i suoi reperti, Ripalimosani (CB), 2006, p.63; J. BARCLAY-BROWN, Face of young saint from the Vestibule area of San Vincenzo, in San Vincenzo Maggiore and its Workshops, R. HODGES, S. LEPPARD, 


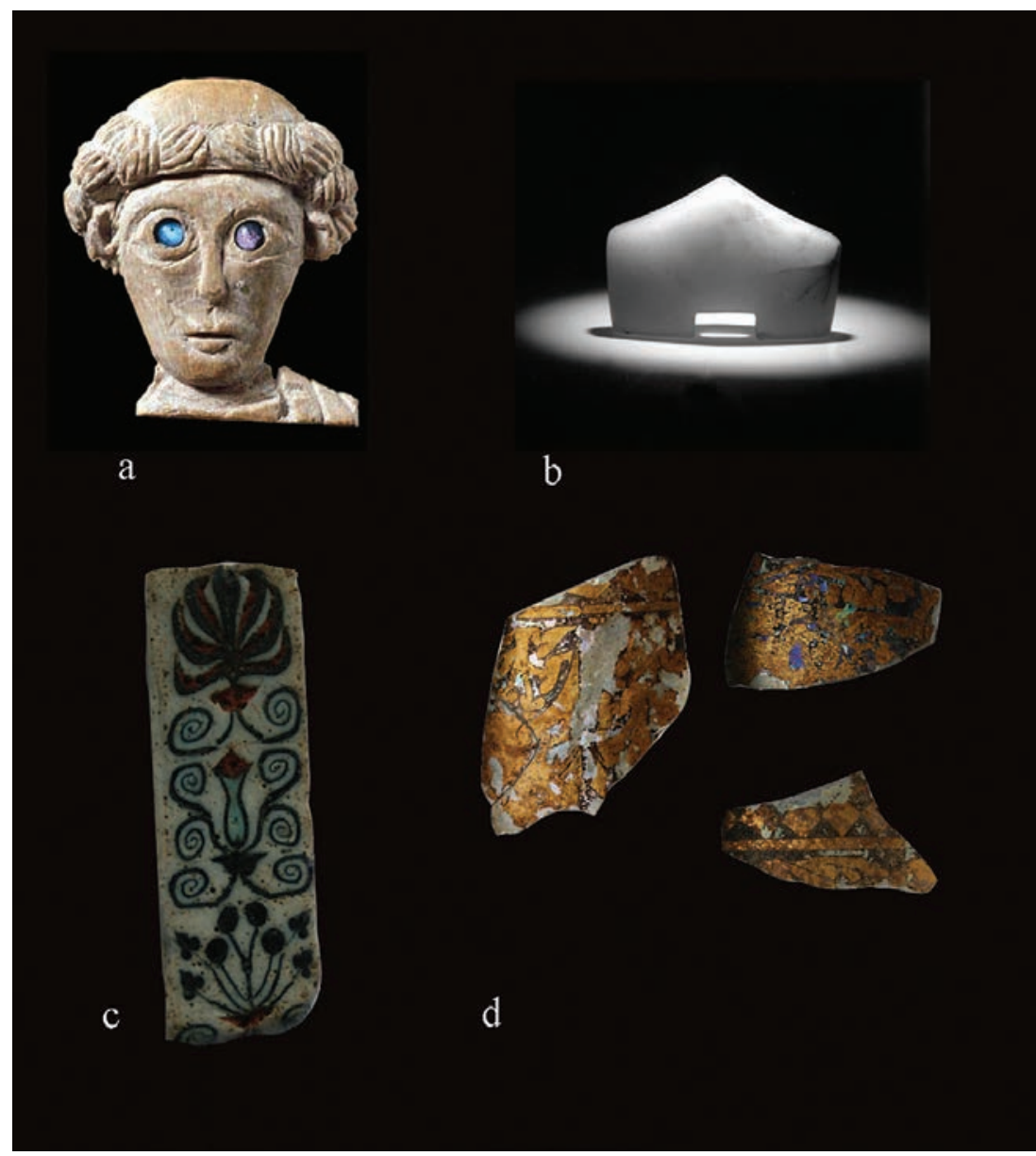

Fig. 12 Oggetti "esotici" dagli scavi di S. Vincenzo: a) testina in avorio; b) elsa di impugnatura da pugnale; c) placchetta in vetro millefiori; d) frammenti vitrei con decorazione a foglia d'oro.

L'altro eccezionale reperto è l'elsa di impugnatura di un pugnale ( $\mathrm{RN}$ 0694) rinvenuta nel giardino porticato (fig.12, b). Si tratta di un pregevole oggetto in nefrite, dalla peculiare forma che ricorda la mitra vescovile ${ }^{63}$. L'eccezionalità del rinvenimento è data anche dalla stessa materia prima, che non risulta estratta in Europa durante l'alto medioevo, e pertanto potrebbe provenire da giacimenti dell'Asia centrale, dove si registrano tra l'altro alcuni plausibili confronti tipologici ${ }^{64}$. Tuttavia l'imprecisione nel taglio della pietra ha suggerito che l'oggetto non fosse opera di un artigiano asiatico. Il rinvenimento di questo reperto suggerirebbe tanto la possibilità di traffici di materie prime - che occasionalmente interessano il nostro monastero - quanto la presenza in loco di artigiani con competenze tecniche tali da poter riprodurre reperti provenienti da aree lontane, in contrasto con le ipotesi precedenti che rigettavano l'idea di scambi culturali ed economici tra la comunità monastica e le aree extraeuropee $e^{65}$. Infine, anche per quanto riguarda la produzionevetraria, lo studio delle officine e delle loro produzioni e l'analisi tipologica dei reperti, suffragata da analisi chimiche dei componenti, suggerisce l'idea di un panorama complesso di scambi ad ampio raggio che consentono a questa, come ad altre abbazie dell'Europa carolingia, un cospicuo approvvigionamento di materie prime ${ }^{66}$. In questo senso il rinvenimento di numerosi frammenti di vetro millefiori e di vetri piani realizzati con metodo "a corona", ha suggerito un'importazione dal Levante, area da cui si ipotizza anche la provenienza di vetrai specializzati che collaborano alla nascita dell'officina vulturnense ${ }^{67}$ (fig.12, c). A questi si aggiunge anche un gruppo di vetri con decorazione a foglia d'oro rinvenuti sia in fase di ricognizione che nella fossa di combustione della fornace centrale (officine temporanee ${ }^{68}$ ). Questi ultimi in particolare ci consentono non solo di ricollegare il monastero di S. Vincenzo alle aree nord-europee, come dimostrerebbero i confronti con oggetti simili di VIII-IX secolo rinvenuti a Dorestad, Helgo e Borg69, ma anche a contesti geografici orientali, dal momento che tali reperti sono considerati imitazione di oggetti islamici ${ }^{70}$ (fig.12, d).

J. MITCHELL (ed.), Rome, 2011, p.268; J. MITCHELL, Saint from the Crypt of Epiphanius, in San Vincenzo Maggiore and its Workshops, R. HODGES, S. LEPPARD, J. MITCHELL (ed.), Rome, 2011, p.269, in cui si ricorda anche una certa attinenza stilistica del pezzo con i volti affrescati rinvenuti in alcuni ambienti monastici, (ma anche con il santo raffigurato nella cripta di Epifanio e i frammenti del volto di santo provenienti dal vestibolo).

${ }_{3}^{6}$ J. MITCHELL, A sword-guard of nephrite, in San Vincenzo al Volturno 3...opc.cit. (n.48), 2001, pp.411-412.

${ }^{64}$ Altri simili reperti in nefrite furono rinvenuti, per il periodo romano a Bystaya (Russia), Chersson (Ucraina) e Orlat (Uzbekistan), mentre per il V secolo si ricorda un reperto nella bassa valle del Volga, altri quattro esemplari a Perm nell'area orientale degli Urali, ed altri ancora nella zona tra Russia e Uzbekistan Cfr. J. MITCHELL, A sword-guard...op.cit. (n.63), 2001.

${ }_{65}$ Tale ipotesi fu avanzata sulla base del confronto con la famosa elsa di spada di Altlussheim di V sec. Cfr. J. MITCHELL, A sword-guard...op.cit. (n.63), 2001. ${ }^{66}$ F. MARAZZI, F. D’ANGELO, Il ciclo della produzione vetraria a San Vincenzo al Volturno nel IX secolo, in Artisanats antiques d'Italie et de Gaule. Mélanges offerts à Maria Francesca Buonaiuto, vol.32, J.P. BRUN (a cura di), Neaples, pp.211-226.

${ }^{67}$ La produzione di vetro millefiori, perfezionata già in epoca romano-imperiale, viene ripresa e semplificata nelle isole britanniche nel corso dei secoli medioevali Cfr. F. DELL'ACQUA, Nisi ipse Daedalus [...] nisi beseleel secundus: l'attività artistica presso il monastero di San Vincenzo al Volturno in età carolingia, in Monasteri in Europa occidentale (secoli VIII-XI), Atti del Convegno Internazionale "Topografia e strutture degli insediamenti monastici dall'età carolingia all'età della riforma, Castel San Vincenzo, 2004, F. MARAZZI, F. DE RUBEIS, (a cura di), Roma, 2008, p. 303. Nello stesso contributo si ricorda poi, che il rinvenimento di pannelli da finestra realizzati tramite il metodo "a corona" proverebbe di per sé un'influenza mediorientale nel bagaglio culturale dei vetrai che operarono a S. Vincenzo. Inoltre, è stato accertato che le officine monastiche utilizzavano rottami di vetro così detto "Egypt II", forse importato dalle coste sud-orientali. Cfr. F. DELL'ACOUA, Mundus habet noctem. Il vetro nelle architetture di Brescia, Cividale, Salerno, San Vincenzo al Volturno, Farfa, in I Longobardi dei Ducati di Spoleto e Benevento. Atti del XVI Congresso Internazionale di Studi sull'Alto Medioevo, Spoleto 2003 , p. 1359.

${ }^{68} \mathrm{Nel}$ primo caso si tratta di tessere in vetro rosso opaco, nel secondo caso di grandi contenitori i cui frammenti erano evidentemente riutilizzati per la produzione di nuovi oggetti. J. STEVENSON, The Vessel glass, in San Vincenzo al Volturno 3...op.cit. (n.62), 2001, p. 274; J. MITCHELL et alii, Le officine di San Vincenzo al Volturno: fasi di produzione e dinamiche di un monastero di IX secolo, in Medioevo: le officine, Atti del Convegno Internazionale di Studi, Parma 22-27 settembre 2009, A. C. QUINTAVALLE (a cura di), Milano, p. 109; S. LEPPARD, J. VICKERY, Gilded glass fragments, in San Vincenzo Maggiore... op.cit.(n.62), p. 263.

${ }^{69}$ Cfr. J. MITCHELL et alii 2008:109; S. LEPPARD, J. VICKERY, Gilded glass fragments, in San Vincenzo Maggiore...op.cit.(n.61), p. 261.

${ }^{70}$ F. DELL'ACQUA, Mundus habet noctem...op.cit. (n.67). 


\section{CONCLUSIONI}

La collazione dei dati provenienti dall'analisi delle proprietà fondiarie monastiche e, nel caso di S. Vincenzo, anche dallo studio delle strutture del recinto/pontile sul Volturno, e dei reperti rinvenuti negli ultimi anni, consente di sintetizzare alcune considerazioni.

La lettura delle fonti documentarie e il laborioso tentativo di riconoscere ed individuare sul territorio $\mathrm{i}$ beni fondiari dei principali monasteri dell'Italia centro-meridionale, sta rivelando un'intricata rete di insediamenti (curtes, casali, castra e celle monastiche), spesso collocati in aree già interessate da antichi scali o passaggi fluviali di epoca romana, miranti ad un serrato controllo delle vie d'acqua, con riguardo particolare per il Volturno. L'area di S. Martino in comune di Ruviano sembra costituire il caso più evidente della combinazione fra lo sfruttamento agrario del territorio e l'accessibilità ai percorsi fluviali che ne possono garantire il più rapido smistamento; ma, lungo il corso del fiume, sono numerose le zone potenzialmente importanti da questo punto di vista e che debbono ancora essere analizzate più approfonditamente.

La presenza di un complesso sistema di banchine lignee e di imponenti strutture di recinzione all'interno del monastero vulturnense, caratterizzate da tecniche edili eredi delle conoscenze del mondo romano, ma riflesso anche di un "know-how" che caratterizza buona parte dell'Europa altomedioevale, costituisce di per sé la prova che tra IX e X secolo, il monastero molisano sia perfettamente in grado di allestire infrastrutture lungo la sponda di un fiume per il carico e scarico di merci e persone.

La concentrazione e quindi la tesaurizzazione di spolia e oggetti suntuari provenienti non soltanto da zone tradizionalmente in contatto con il monastero, come Capua, ma anche da aree piuttosto lontane del Nord Europa e del bacino mediterraneo, dimostrerebbe abbastanza chiaramente come questo network, probabilmente nato in origine per coprire un raggio d'azione "regionale", raggiunga il suo apice proprio in epoca carolingia, in concomitanza con il fiorire dei maggiori snodi portuali europei e il rilancio di quelli mediterranei. A tal proposito ricordiamo che tanto S. Vincenzo quanto Montecassino rafforzano il proprio quadro economico con l'acquisizione di beni, scali e diritti di pesca legati non solo ai laghi campani come nel caso di lago Patria ma anche a quelli pugliesi come ad esempio il lago di Lesina ${ }^{71}$. Certamente non possiamo credere che un tale flusso di merci possa espletarsi esclusivamente attraverso la rete fluviale, ma possiamo immaginare che quest'ultima venga "messa a sistema" con la rete di porti marittimi che dal Mediterraneo e dall'Adriatico consentono contatti con il Nord Europa. Tale operazione tradisce probabilmente l'intento di costituire un sistema integrativo a quello viario tradizionale, poiché quest'ultimo nell'altomedioevo può risultare a volte poco agevole e certamente molto dispendioso.

\footnotetext{
${ }^{71}$ Nel caso di lago Patria, ricordiamo il diploma dei principi Landolfo e Pandolfo del 958, in cui si concede a S. Vincenzo il diritto di pesca con la facoltà di inviare una paranza ("paratura") costituita da due barche a remi ("bontris") e quattro pescatori (CV II, 125). Cui segue una concessione di curtes e terre presso il Lago patria in favore di Montecassino nell'agosto del 1052 (CMC II, 83).

Per quanto concerne il lago di Lesina il Chronicon ricorda che tra i beni del monastero, acquisiti intorno all'8oo, c'era oltre alla foce del lago, anche un'eccellente peschiera (CV I, 34). Le terre in questione, con i relativi diritti di pesca saranno cedute tramite livello nel 965 (CV II,131) e successivamente oggetto di giudicato nell'980 (CV II: 132, cfr. anche CMC II,2 in cui compare tanto la peschiera quanto la foce sul lago; CMC II,8; per l'anno 981; CMC II, 13 per l'anno 987; CMC II, 26 per l'anno 1047). La Chronica Monasteri Casinensis nell'899 annota un simile dato anche per la peschiera di Montecassino (CMC I,48). Questi documenti tra l'altro testimoniano anche la prassi, piuttosto diffusa nell'altomedioevo, delle concessioni di porti, flotte e sgravi fiscali agli enti ecclesiastici (cfr. G. FASOLI, Navigazione fluviale. Porti e navi sul Po, in La navigazione medietarranea nell'alto medioevo, Spoleto, 1977, pp.566-6o7; M. MIDDLETON, Early medieval port customs, tolls and controls on foreign trade, in Early medievale Europe, vol.13, Issue 4, 2005, pp.313-358).
} 


\section{PORTI FLUVIALI E PONTI IN ETÀ MEDIEVALE. IL PO E L'AREA PADANA. ROBERTO GRECI}

UDC: $711.453 \cdot 4-167(282.245 \cdot 13) " 04 / 14 "$

Original scientific paper

Manuscript received: 29. 03. 2016.

Revised manuscript accepted: 22. 04. 2016.

DOI: 10.1484/J.HAM.5.111346
R. Greci

Dipartimento di Lettere, Arti, Storia e Società Via Massimo d'Azeglio 85, 43125 Parma

Italia

During the Middle Ages the Po Valley garantees the continuity of trade and the progress of the economy of northern Italy. The sources tell us that many river ports and docks were useful to the manors of monasteries and churches, holders of public rights. These sources also allow to identify some significant trends: the progressive expansion of a modular system of waterways, even if the navigation is impossible today (see the case of Bologna), and the growing involvement of the neighboring towns into the river system (Pavia, Piacenza, Cremona, Mantova and so on). Outside the cities, the castles and the parish churches on the river ports attest that the ports attract settlements and territorial powers (Canossa). Furthermore, the coincidence of ports and ponti (i.e. ferries for river crossings) promotes the vitality of the roads; the stretch of the river between Cremona and Parma, for example, is an important meeting point between Po river and the road of Monte Bardone, which was the favourite road for the Lombards to reach Tuscany through the Appennines.

Keywords: High Middle Ages, Po Valley, River ports

La storiografia italiana, negli ultimi anni, si è concentrata in maniera significativa sullo studio della viabilità medievale. L'interesse non è stato rivolto solo alla ricostruzione dell'andamento e delle trasformazioni degli itinerari, ma ha portato anche alla elaborazione di una categoria interpretativa dei fenomeni storici connessi, quell' "area di strada", che bene indica lo stretto rapporto tra viabilità e vocazioni (economiche, politiche e culturali) di certe regioni o di certe zone. A fronte di una maggiore attenzione alla viabilità terrestre, come anch'io in passato ho fatto avviando una serie di ricerche dedicate all'Emilia occidentale all'interno di un progetto di ricerca europeo, una minore attenzione è stata dedicata alla viabilità fluviale e alle strutture delle comunicazioni che la viabilità fluviale consentiva ${ }^{1}$. Le ragioni di questo sono da riconoscersi in almeno due cause. Prima di tutto si ritiene comunemente che in età medievale si sia passati da una prevalente "fluvializzazione dei trasporti" altomedievale (la definizione, risalente a Roberto Sabatino Lopez fa riferimento soprattutto all'ambito economico) ad una crescente e inarrestabile prevalenza della viabilità terrestre nel basso medioevo a seguito di una più stabile capacità di coordinamento delle strutture politiche, del miglioramento degli strumenti tecnici, del capillare ampliamento delle reti di rapporti. In secondo luogo (e per conseguenza) si ipotizza il depotenziamento di strutture materiali, che però, almeno in parte, può essere l'effetto di una deformazione prospettica che spesso pesa nell'osservazione del passato. Eppure le fonti evidenziano che ambienti lagunari e fluviali non sono spazi vuoti ma sono un terreno fecondo per la ricerca storica e archeologica, quest'ultima, in realtà, più difficile e fortemente condizionata dalla casualità.

Non v'è dubbio che l'importanza delle comunicazioni fluviali rimane viva per tutta l'età medievale, specie in quelle zone in cui la presenza di corsi d'acqua di una certa portata continuva a rendere più agevoli e più economici comunica- zioni e trasporti. Una di queste zone, una vera e propria "area di fiume" (mutuo il concetto dall"'area di strada") è l'intera Pianura Padana che si configura come un sistema complesso comprendente il Po, spina dorsale dell'insieme, ma anche la rete fitta di corsi d'acqua ad esso collegati. Area di fiume, quindi e non solo una sommatoria di luoghi di fiume perchè ritengo che si possa cercare di guardare al fenomeno in una prospettiva ampia che superi (senza necessariamente escluderlo) il descrittivismo minuto; il che implica, beninteso, un ampio lavoro di censimento e di catalogazione che sarebbe necessario portare avanti. Le ragioni dell'abbinamento porti e ponti conenuto nel titolo di questo intervento, infine, risiede nella possibile sovrapposizione delle due realtà; con il termine portus, infatti, le testimonianze possono alludere anche (o solo) all'imbarco del traghetto per l'attraversamento del fiume, una funzione che in molti casi coincide, quindi, con quella propria del ponte generando frequenti incertezze nella lettura delle testimonianze.

Il fiume Po, per buona parte navigabile in età antica (quantomeno da Pavia, ma secondo alcuni autori antichi perfino da Torino) è rimasto anche nell'alto Medioevo un'arteria di comunicazione continuativamente sfruttata, non foss'altro perchè poteva congiungere agevolmente due città fondamentali nella politica del tempo, Pavia e Ravenna. In questa sede vogliamo rimarcare il collegamento tra il fiume, i centri economici e i centri urbani più o meno prossimi al suo corso; questi ultimi poterono avvantaggiarsi di questa vicinanza, alimentando le proprie economie e garantendo ai territori interni di aprirsi al commercio a distanza superando le crisi conseguenti ai rivolgimenti del primo medioevo e determinando uno sviluppo più rapido e consistente rispetto a quelli di altre aree italiane. Il Po, insomma, ci appare come l'asse portante di un sistema ben più vasto e complesso se consideriamo l'ampio spettro dei suoi affluenti, che diversamente da oggi venivano utilizzati per la navigazione talora

${ }^{1}$ R. Greci, a c. di, Un'area di strada: l'Emilia occidentale nel Medioevo. Ricerche storiche e riflessioni metodologiche, Bologna 2000. 
proseguendo anche in questo caso l'eredità romana, e la percorribilità di un sistema stradale sempre vivo, pur nella variabilità dei suoi percorsi. ${ }^{2}$

E' noto che Sidonio Apollinare, nel suo viaggio da Lione a Roma (V secolo), utilizzò il Po per attraversare, su una nave cursoria, la Gallia Cisalpina. Il percorso tra Pavia e Ravenna, in età tardo-antica, era ancora bene organizzato; scanditodalle tappe del servizio postale, si articolava in due tratti e tra il primo (Pavia, Cremona, Brescello) e il secondo (Ostiglia, Voghenza, Ravenna) era prevista una regolare sostituzione dei battellieri. La vasta rete idroviaria romana era costituita da un sistema integrato tra le aree lagunari adriatiche (da Aquileia a Ravenna), il Po e i flumi che rendevano permeabile la zona continentale. L'Adige, ad esempio, favoriva i collegamenti della costa con Verona, ma anche col Trentino fino alle vicinanze di Bolzano (Bronzolo), mentre altri affluenti avvicinavano il basso corso del Po ai laghi e ai valichi alpini sostenendo il trasporto di merci quali vino, legname, mar$\mathrm{mi}$, troppo pesanti e ingombranti per circolare sulle strade (Emilia, Postumia, Gallica). Da Ostiglia ci si poteva dirigere verso il Nord tramite Mantova, Peschiera, lago di Garda e poi, attraverso la valle del Sarca, raggiungere Trento e le Giudicarie; itinerari su cui agivano quei battellieri veronesi (di Mantova e Peschiera) e bresciani (a Sommolago) organizzati in corporazioni. L'età romana ci ha lasciato inoltre memoria di canalizzazioni pensate per agevolare la navigazione fluviale sempre soggetta ai rischi dei bassi fondali: ad esempio la fossa aperta nel Parmense da Emilio Scauro nel 109 a.C. e quella augustea del Piacentino (poi denominata Fodesta), così come la ben più importante Fossa Augusta che doveva collegare, tramite il vecchio corso del Po (Padusa), il porto di Ravenna con l'asse padano. L'area padana ha lasciato inoltre testimonianze archeologiche di attrezzature portuali. Tali sono le banchine del porto fluviale di Padova da cui si dipartivano ripide rampe verso la carreggiata che costeggiava il fiume e verso la piazza del mercato con magazzini porticati; o le più semplici ma non meno significative strutture di Sirmione e di Padenghe sul Garda, costituite da semplici moli di legno aggettanti nel lago, da ormeggi in pietra e da bitte. Ci ha lasciato anche traccia di vari tipi di imbarcazioni funzionali al particolare tipo di navigazione che i fiumi padani potevano sostenere: quelle zattere (rates), piroghe, monoxyla rinvenute nel Bacchiglione, nel Brenta ea Comacchio nel Ferarrese cui dovevano affiancarsi le più fragili (e quindi scomparse) imbarcazioni di vimini e pelli (carabus) o quelle specie di sandali e burchielli di fasciame e a fondo piatto citati dalle fonti scritte (Tito Livio). Nelle città, presso i porti, dovevano forse essere ubicate infrastrutture simili agli horrea di Aquileia, Padova, Ostiglia, Piacenza, Pavia. ${ }^{3}$.

Questo assetto con le infrastrutture necessarie al suo funzionamento dovette subire un sensibile degrado nell'alto Medioevo a seguito delle peggiorate condizioni economiche e delle non lievi trasformazioni a seguito della frammentazione politica che rendeva evanescente ogni forma di coordinamento. Infatti, se si esclude l'età longobarda, per la quale, come vedremo, abbiamo notizia di un interessamento pubblico alla navigazione padana, il periodo successivo è una progressiva tendenza alla frantumazione dei poteri che culminerà con l'affermazione delle autonomie cittadine nell'età comunale. Questo quadro deve essere tenuto presente anche ai nostri fini perché l'assenza di un'autorità pubblica dovette implicare l'assenza di una sistematica regolamentazione progettuale ${ }^{4}$, particolarmente indispensabile per i fiumi e per il Po in particolare, il quale, per la sua natura ondivaga, ha sempre richiesto forme di manutenzione (argini, consolidamento di sponde, bonifiche) che ne garantissero la complessiva funzionalità'. Il protagonismo del potere pubblico nella sorveglianza del corso del fiume e delle sue rive, considerate demaniali quanto al loro uso, era un'eredità che affondava nel diritto romano ${ }^{6} \mathrm{e}$ che si proiettava nei regni romano-barbarici e anche oltre quando, in maniera indiretta, gli utenti avrebbero continuato nell'obbligo di fornire prestazioni in natura o in denaro a chi avrebbe sostituito il pubblico nel possesso delle diverse parti del fiume.

Se ancora durante la guerra greco-gotica la via fluviale padana appare intensamente frequentata, successivamente non mancarono di farsi sentire le conseguenze destrutturanti del conflitto longobardo bizantino: $\mathrm{i}$ blocchi delle foci e la conquista e la distruzione di Cremona e di Brescello da parte di Agilulfo furono indubbiamente fattori potenzialmente destrutturanti. L'interesse per il Po delle fonti normative, tuttavia, attesta come esso continuasse ad essere percepito come elemento indispensabile per il Regno il cui territorio si estendeva per buona parte al di qua e al di là del fiume. Le leggi longobarde, che pure non vanno oltre la menzione della figura del portonarius incaricato di impedire il transito di ladri e schiavi fuggitivi, lasciano intravvedere l'esistenza di porti, ovvero di ponti per l'attraversamento, quanto meno funzionali ad un traffico locale.

Nella situazione politica bipartita che si era creata, non erano più possibili progettualità ed interventi globali, a causa della compresenza dei bizantini su una parte del corso del Po, quella terminale, essenziale per gli scambi a lunga distanza. Ma, nel progressivo assestamento della nuova situazione, la via fluviale padana dovette rapidamente rianimarsi. Oltre alle notizie dell'esistenza di competenze tecniche nel regno longobardo ricercate, a detta di Paolo Diacono, dagli Avari nell'età di Agilulfo (primi anni del VII secolo), abbiamo soprattutto la eccezionale testimonianza dell'età liutprandea, una testimonianza di natura fiscale, indice di un assestamento amministrativo sul fronte degli scambi non velleitario e contingente, se potrà lasciare tracce ancora nel X secolo, a dar credito alle Honorantiae civitatis Papiae, un testo dell'XI che però rifletteva situazioni precedenti 7 .

\footnotetext{
${ }_{2}^{2}$ L. CRACCO RUGGINI, Economia e società nell"'Italia annonaria", Milano, 1961, p. 285

${ }_{3}^{3}$ Per tutte queste notizie, G. UGGERI, Aspetti archeologici della navigazione interna nella Cisalpina, in Aquileia e l'Alto Adriatico, (Antichità altoadriatiche, XXXVI), Udine, 1990, p. 175-196. Le semplici imbarcazioni (chiatte) a fondo piatto continueranno a popolare fiumi e porti in età medievale: G. FASOLI, Navigazione fluviale. Porti e navi sul Po, in Navigazione mediterranea nell'alto Medioevo. Settimane di Studio del Centro Italiano di Studi sull'Alto Medioevo, 25, 14-20 aprile 1977, 2, Spoleto, 1978, p. 565-607, a p. 576 s.

4 P. RACINE, Poteri medievali e percorsi fluviali nell'Italia padana, in Quaderni storici, 31, 1986, p. 9-32, p. 9.

${ }_{5}^{5}$ M. DI GIANFRANCESCO, Per una storia della navigazione padana dal Medioevo alla vigilia del Risorgimento, in Quaderni storici, 28, 1975, p. 199-226. Per un rapido sguardo all'area emiliana, vd. R. GRECI, Vie di comunicazione e mezzi di trasporto nel Medioevo, in Cultura popolare dell'Emilia Romagna, Le origini e i linguaggi, Cinisello Balsamo (Mi), 1982, p. 205-226

${ }^{6}$ Digesto, 43, 12-15.
} 
Il famoso patto di Liutprando del 715-730, infatti, attesta il traffico tra Comacchio e l'interno della Langobardia che vedeva il monopolio dei milites comacchiesi nel trasporto e nel commercio del sale prodotto nel delta e destinato ai porti rivieraschi padani, sedi di ufficiali (riparii) deputati a riscuotere imposte per l'attracco delle navi e per lo scarico e la vendita delle merci: il porto mantovano, il porto in capo Mincio, quello bresciano, il porto qui vocatur Cremona, quello qui appellatur Parmisiano, il portus qui dicitur Addua e quello qui dicitur Lambro et Placentia. Il diploma cui ci riferiamo è ben noto ed è stato oggetto di attenzione a partire dagli studi di Cinzio Violante fino al successivo e più organico studio di Gina Fasoli ${ }^{8}$. La solidità e la durata della situazione ascrivibile all'VIII secolo è confermata dagli studi archeologici condotti sul sito di Comacchio. Iniziati negli anni Venti e intensificati negli anni Novanta del secolo scorso, gli scavi hanno evidenziato strutture importanti, frutto di investimenti e di progettualità significative. Palificazioni portuali, piattaforme lignee di maggiore e minore entità sostenute da ordinate file di grandi pali verticali che fanno pensare a moli e a spazi di lavoro per il carico, scarico e smistamento merci, ma anche costruzioni lignee destinate ad ospitare magazzini o abitazioni. Le strutture, che sfruttavano la presenza di un canale artificiale forse realizzato nell'alto medioevo e di un bacino lagunare, si presentano in un contesto dotato di un abitato centrale (ubicato dove oggi c'è la cattedrale) e di un abitato sparso pure esistente. Tale complessa realtà fa bene intendere l'importanza di questo scalo per i rapporti con Ravenna a sud (possibili grazie ai percorsi lagunari), con il Po e le città padane (a monte) e con l'Adriatico e Mediterraneo a est 9 ; un'importanza che si ridusse solo alla fine del X secolo a seguito della comparsa nel traffico fluviale di Venetici, Ferraresi, Cremonesi e Pavesi, e alla concorrenza, nella zona lagunare, dei porti di Goro e di Volano, spettanti al monastero di Pomposa e alla chiesa ravennate e punti obbligati per l'accesso alle rotte marittime ${ }^{10}$.

Quanto finora detto consente alcune riflessioni sul ruolo dei porti fluviali padani nell'alto Medioevo. Innanzitutto le testimonianze di età longobarda ci mostrano come il tono economico di quell'età non fosse totalmente avvilito e, in particolare, come le fratture politiche non avessero determinato una totale interruzione degli scambi; e tutto ciò proprio grazie alla continuità della funzione del Po, dei suoi porti, dei suoi punti di attraversamento. Il patto di Liutprando ai Comacchiesi ci offre inoltre (in assenza di una cartografia) la possibilità di abbozzare la mappa di un sistema coerente di porti funzionanti nella parte longobarda del corso del fiume, quella che qui più ci interessa. Tale mappa non può essere completa e puntuale per tutta una serie di ragioni: perché essa è limitata agli scambi coi Comacchiesi e agli interessi fiscali di età liutprandina, perché le località in essa considerate non trovano facili riscontri nella toponomastica odierna, perchè il corso del Po è sensibilmente e continuamente mutato nel corso dei secoli manifestando una incessante provvisorietà, perché le strutture materiali dei siti portuali infine, elementari e fragili, sono scomparse senza lasciare tracce individuabili con una superficiale analisi del territorio, peraltro anch'esso assai trasformato. Ciononostante rileveremo che il documento, non indicando le località portuali con nomi specifici e diversi, fa riferimento - per lo più - alle città, sia che queste ultime non fossero vicinissime agli scali (è il caso bresciano, mantovano, parmense) sia che vi fosse coincidenza, anche di nome, tra le une e gli altri (Piacenza e Cremona, quest'ultima in realtà vistosamente decaduta, dopo la distruzione del 603, dal rango di città ma evidentemente rapidamente rivitalizzata grazie alla funzionalità del suo porto). Quei porti poi che non fanno riferimento al territorio cittadino o ad un centro urbano vengono semplicemente collocati nel punto geografico in cui alcuni fiumi navigabili (Adda, Lambro) confluiscono nel Po evidenziando la loro primaria finalità di snodi nei trasporti del tempo.

Con l'eccezione di Cremona e Piacenza abbiamo dunque l'impressione di trovarci di fronte a strutture dislocate in contesti privi di insediamenti significativi, capaci di assorbire lo scalo dando ad esso il nome o generando un toponimo comprensivo anche dell'insediamento. Se ciò è vero, dobbiamo presupporre una attenzione particolare dell'amministrazione pubblica verso zone diverse dai tradizionali centri urbani, condizionati da incerte vicende istituzionali e quindi meno facilmente controllabili. La loro importanza anche per la città aveva comunque la capacità di condizionare o di sfruttare la viabilità circostante e perfino di condizionare la collocazione di enti monastici cittadini orientando l'assetto viario dello stesso centro urbano; ciò appare nel caso di Parma, città in cui, in età carolingia, si notano strategie di collegamento viario tra monasteri, ma anche chiesa, cittadini e scalo fluviale ${ }^{11}$. L'esistenza di questa sequela di scali e i non interrotti legami con le città, dunque, alimentano ipotesi sempre meno pessimistiche sulle condizioni economiche del regno longobardo e, più in generale, sul problema della continuità e della frattura tra tardo antico e alto medioevo. Tuttavia, al di là di una idea di continuità limitativa se incentrata esclusivamente e banalmente sul ruolo del centro urbano, è presumibile immaginare che questi scali costituissero punti di recente sviluppo o località degne di potenziamento da parte dei poteri pubblici; cer-

\footnotetext{
${ }_{7}$ C. BRÜHL, Das "Palatium" von Pavia und die "Honorantiae civitatis Papiae", in Pavia capitale di Regno, Atti del IV Congresso internazionale di Studi sull'Alto Medioevo, Pavia - Scaldasole - Monza - Bobbio, 10-14 settembre 1967, Spoleto, 1969, p. 189-220.

${ }^{8}$ C. VIOLANTE, La società milanese in età precomunale, Bari, 1953; G. FASOLI, op. cit. (n. 3); per un confronto tra situazione italiana e francese, si veda J. ROSSIAUD, Les ports fluviaux au Moyen Âge (France, Italie), in Ports maritimes et ports fluviaux au Moyen Age. Actes des Congrès de la Société des historiens médiévistes de l'enseignement supérieur public, 35e congrès, La Rochelle, 5-6 Juin 2004, Paris, 2005, p. 9-19.

${ }^{9}$ S. GELICHI, Infrastrutture marittime nell'alto medioevo: una prospettiva archeologica, in L'acqua nei secoli altomedievali, Settimane di Studio del Centro italiano di Studi sull'Alto Medioevo, LV, Spoleto, $12-17$ aprile 2007, 1, Spoleto, 2008, p. 283-317, p. 310 s. Ma per Comacchio si veda anche S. GELICHI, E. GRANDI, Comacchio: una città alto-medievale e l'archeologia, Faenza, 2013.

${ }^{10}$ G. FASOLI, op. cit. (n. 3) pp. 602-603.

"Parma è distante dal Po, ma sul Po c'è il portus parmisianus e la città, attraversata dal torrente Parma e vicina al fiume Taro, entrambi convergenti sul Po, risente del bisogno di questo importante collegamento. I principali enti religiosi cittadini, proprietari di curtes, si collocano in prossimità del torrente su cui sorge il centro urbano sia per usufrire del collegamento fluviale con il Po, sia per usufruire della viabilità terrestre che li vedeva terminali di strade tendenti verso il Po; R. GRECI, L'economia urbana, in R. Greci (dir.), Il Medioevo. Economia, società, memoria, in D. Vera (dir.), Storia di Parma, III/2, Parma, 2011, p. 107-158.
} 
tamente non avulsi dalla capacità attrattiva della città, ma tendenzialmente funzionali all'emergere di diversi flussi di interessi determinati dall'emersione, tra VIII e IX secolo, di nuovi leader economici e di nuovi assetti patrimoniali proiettati, grazie ad una rete di scali più diffusa e meno rigida, in uno spazio più ampio, non univocamente convergente sulla città. Assetti patrimoniali che non escludevano, in quanto sedi degli enti privilegiati, i centri urbani, alcuni dei quali avrebbero infatti sentito il bisogno, già alla fine dell'XI secolo e preannunciando tendenze di età comunale, di diventare protagoniste nel controllo del fiume progettando la costruzione di appositi canali navigabili: è il caso dei Modenesi che avrebbero ottenuto l'autorizzazione dall'imperatore Enrico IV di aprire un navigium usque in Padum per incrementare i commerci con Venezia, Ravenna ed altri porti ${ }^{12}$.

Non v'è dubbio insomma che il commercio fluviale fosse un fattore di crescita anche per la città; ma è pur vero che il il ruolo dell'organizzazione e dell'economia curtense appare trainante e soprattutto capace di inserire centri urbani diminuiti di importanza in una rete di rapporti e di interessi esterni alla città, indubbiamente più ampi e meno occasionali, nella disarticolazione del sistema precedente. Esemplificativo è il caso di Piacenza in cui si misura il trapasso da una fase in cui il potere pubblico continuava a rapportarsi genericamente alla città ad una fase in cui andavano emergendo soggetti concorrenziali e alternativi, sia rispetto al godimento dei diritti pubblici sia rispetto al ruolo economico del centro urbano. Ciò lo si evince dalla concessione di diversi diritti sul fiume che il re Astolfo fece - oltre che all'abate di Nonantola - al vescovo di Piacenza (che già nel 744 prelevava una somma dalle navi che approdavano nel porto cittadino), ma anche da quella che, negli ultimi anni del Regno, il re Desiderio fece al monastero di San Salvatore di Brescia, di cui era badessa la figlia Anselberga, riguardante i diritti regi sul porto e ponte sul Po di Piacenza. Possiamo supporre l'affermazione di una politica bifocale, disponibile ad affrontare in modo pragmatico le situazioni a fronte di un indebolimento delle strutture statali e dei centri urbani, pronta ad usare i diritti pubblici in maniera non tradizionale operando consapevoli distinzioni: il traffico a lunga distanza e "internazionale" tendeva a restare sotto il controllo pubblico (secondo i privilegi liutprandini nella continuità del monopolio dei Comacchiesi), mentre il traffico locale e regionale tendeva a far capo ad enti che usavano sistema portuale e navigazione padana come supporti ai propri interessi particolari, gravitanti intorno alle numerose curtes disseminate in luoghi tra loro anche molto distanti ${ }^{13}$. E' vero che i nuovi protagonisti, come si è detto, sono spesso enti cittadini, eredi di quel legame città/porti in parte ancora sancito dal privilegio di Liutprando; ma è da notare che le concessioni, non necessariamente riguardanti il porto della città cui appartiene l'ente privilegiato, se da un lato potevano indebolire gli interessi (vescovili) locali, dall'altro aprivano lo scalo cittadino a rapporti molteplici. Il monastero di Bobbio, ad esempio, lontano dalla città ma ad essa collegato dalla Valle del Trebbia, era bisognoso della via fluviale per i suoi ampi interessi padani: sull'Adda il monastero aveva uno xenodochio a Castel Lupano, dove era funzionante un porto fluviale; le relazioni coi beni ubicati "in Cremonensibus" convergevano inoltre sul porto di Cremona, punto di confluenza di antiche strade ${ }^{14}$, mentre il Porto di Mantova (ove il monastero riscuoteva tributi dalle naves venetice e da quelle comacchiesi) e il Mincio risultavano fondamentali per i collegamenti del monastero coi beni posseduti sul Garda (così come, più ad est, i collegamenti coi possessi del Veronese erano assicurati dalla frequentazione di Adige e Brenta). Per questa ampia proiezione dei propri interessi sparsi nell'Italia settentrionale e presenti anche a Comacchio dove forse possedeva saline, Bobbio poteva contare sul libero transito delle sue navi sul Po e sul Ticino (a Pavia teneva stabili punti di vendita presieduti da un monaco e non da semplici servi) grazie ad antichi privilegi che sarebbero stati continuativamente confermati in età carolingia (860), berengariana (903) e ottoniana (972) ${ }^{15}$. Si trattava di esenzioni assai consistenti ma che indubbiamente moltiplicavano il ruolo dello scalo fluviale piacentino e delle sue strutture.

Parimenti i privilegi di un monastero non piacentino, come quello di San Salvatore/Santa Giulia di Brescia, indebolivano gli interessi pubblici o vescovili su di esso, ma nel contempo garantivano un vantaggioso incremento del traffico che alla città faceva capo. Questo convergere sul centro urbano di interessi esterni, connessi alla frantumazione del controllo pubblico determinò una situazione poco chiaramente riflessa nelle fonti scritte che parlano del porto (o piuttosto dei porti) di Piacenza. Il moltiplicarsi di concessioni rende infatti problematica l'individuazione e la collocazione dello scalo fluviale piacentino. Tali concessioni, rispetto a preesistenti strutture di pertinenza pubblica, implicavano la effettiva realizzazione di strutture distinte o aggiuntive (vista la semplicità di allestimento degli approdi) oppure si limitavano, più banalmente e immaterialmente, alle esenzioni di prelievi fiscali connesse all'uso plurimo delle stesse strutture? In età carolingia, quando pure il potere pubblico continuava ad esigere dai mercanti le antiche imposte "tam de pontibus et de navigiis" (805), continuarono le conferme e le nuove alienazioni di diritti pubblici: l'imperatore Ludovico II, infatti, concesse al monastero - in questo caso cittadino - di San Sisto (badessa la moglie Angilberga), non solo possessi, ma anche diritti di navigazione e un porto su Po-Adda ad essi collegati (862). Il quadro che uscì da questa politica empirica, meno attenta ai proventi fiscali ma di fatto sensibile agli interessi dei molteplici protagonisti della vita economica, dovette realmente determinare una

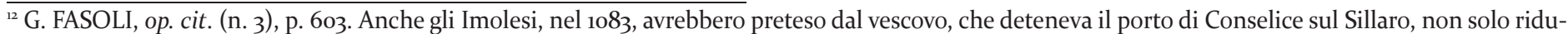
zioni di tariffe, ma anche l'apertura di un canale che avvicinasse al centro urbano il porto, distante ben 18 chilometri

${ }^{13}$ P. RACINE, op. cit. (n. 4), p. 12; anche nella Francia monasteri, palazzi regi di età carolingia e città sono sui fiumi o nele loro vicinanze o nei loro foci: R.-H. BAUTIER, La circulation fluviale dans la France médiévale, in Recherches sur l'économie de la France médiévale. Les voies fluviales. La draperie, Paris, 1989 , p. 7-36.

${ }^{14}$ F. SOLDI, La capitale del Po, Cremona, 1957.

${ }^{15}$ La concessione di Ludovico II dell'86o fu confermata da Berengario I nel 903 (I Diplomi di Berengario I, a c. di L. Schiaparelli, Roma, 1903, n. XL, p. 115) e da Ottone I nel 972 (Codice diplomatico del monastero di San Colombano di Bobbio fino all'anno 1208, a c. di C. Cipolla, I, Roma, 1918, n. XCVI, p. 325). Per la presenza di Bobbio nel Mantovano, S. Colombano di Bobbio, in A. Castagnetti et al. (dir.), Inventari altomedievali di terre, coloni e redditi, Roma, 1979, p. 113-118. Su Bobbio, E. Destefanis, P. Guglielmotti (dir.) La diocesi di Bobbio. Formazione e sviluppi di un’istituzione millenaria, Firenze, 2015.
} 